\title{
Systems Modeling and Intelligent Control of Meat Drying Process
}

\author{
by \\ Hong Ma \\ A Thesis \\ presented to \\ The University of Guelph
}

In partial fulfillment of requirements

for the degree of

Master of Applied Science

in

Engineering

Guelph, Ontario, Canada

C)Hong Ma, September, 2015 


\section{ABSTRACT \\ SYSTEMS MODELING AND INTELLIGENT CONTROL OF MEAT DRYING PROCESS}

\section{Hong Ma}

University of Guelph, 2015
Advisor:

Professor Simon X. Yang

The main objectives of this thesis are to develop a system model and an intelligent controller for the meat drying process, which can provide better control accuracy than the proportional-integral-derivative (PID) control system. In a meat drying room, important environment variables of temperature and relative humidity are coupled by a nonlinear relationship. Therefore, the design of a nonlinear, intelligent controller is required.

The meat drying room system model encompasses both the plant model and the coupling model between temperature and relative humidity. The proposed intelligent controller uses a fuzzy PID control method and a PI-like fuzzy logic control method to limit the fluctuations in the meat drying system. Results show that the performances of the proposed fuzzy PID and PI-like fuzzy control systems are superior in terms of relative humidity control accuracy. 


\section{Acknowledgements}

First and foremost, I would like to acknowledge the contribution of all people who provided encouragement and invaluable help for accomplishing this thesis.

I would like to express my gratitude to my advisor, Professor Simon X. Yang, for his invaluable guidance, assistance, and encouragement throughout my graduate study at the University of Guelph. His constructive comments and masterly guidance during the writing of this thesis are greatly appreciated. I also wish to thank my advisory committee member, Dr. Mohammad Biglarbegian for his guidance, assistance, and encouragement. I wish to thank Dr. Shohel Mahmud for his assistance.

The school of engineering has provided me with a research environment that supportive to completing this thesis. I would like to take this opportunity to thank all of my colleagues and friends and express my sincere appreciation for their help, encouragement, and support in the past three years.

The writing services department of the library has provided me with a great support for proofreading in this thesis. I would like to take this opprtunity to express my appreciation to everyone who worked with me for their help and support.

The great acknowledgement would be given to my dear dad and mom. Without their support, encouragement, foresight, and sagacity in my hard childhood, I would not have succeeded today.

Finally, I am very indebted to my husband, Wei, and son, Lei, for their dedication and support. 


\section{Contents}

List of Figures $\quad$ vii

List of Tables $\quad x$

List of Symbols $\quad$ xi

List of Abbreviations $\quad$ xvi

1 Introduction 1

1.1 Problem Statement . . . . . . . . . . . . . . . . 2

1.2 Objectives of This Thesis . . . . . . . . . . . . . 4

1.3 Contributions of This Thesis ................ 5

1.4 Organization of This Thesis . . . . . . . . . . . . 6

2 Background and Literature Survey $\quad 8$

2.1 Temperature and Relative Humidity Measurements . . . . . . . . . . . . 8

2.1.1 Measurement Methods . . . . . . . . . . . . . . 9

2.1.2 Psychrometers ...................... 12

2.2 Systems Modeling . . . . . . . . . . . . . . 16

2.2.1 Continuous-Time Models . . . . . . . . . . . . 17

2.2.2 Discrete-Time Models . . . . . . . . . . . . . . . 18

2.2.3 Systems Modeling from Experimental Data . . . . . . . . . . . . 19

2.3 Coupling Models . . . . . . . . . . . . . . . 20

2.3.1 Fuzzy Logic Based Models . . . . . . . . . . . . . . . . . 21 
2.3.2 Neural Network Based Models . . . . . . . . . . . . . . . . 23

2.3.3 Adaptive-Network-Based Fuzzy Inference Systems . . . . . . . . 26

2.4 Control Methods for Temperature and Relative Humidity . . . . . . . . . 28

2.4.1 Temperature and Relative Humidity Control . . . . . . . . . . . 29

2.4.2 Analog Control Systems _ . . . . . . . . . . . . . . . . 31

2.4.3 Digital Control Systems _ . . . . . . . . . . . . . . 31

2.4.4 Fuzzy Logic Control for Temperature and Relative Humidity . . . . 32

2.5 Energy Concerns for Meat Drying Systems _ . . . . . . . . . . . 35

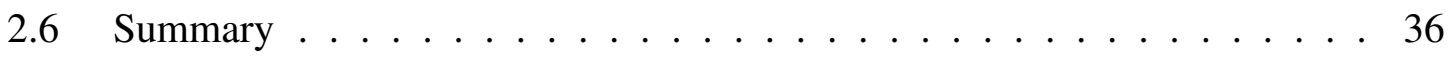

3 The Proposed Models for Meat Drying Process 37

3.1 Introduction $\ldots \ldots \ldots \ldots \ldots \ldots \ldots \ldots \ldots \ldots \ldots$

3.2 The Proposed Models _ . . . . . . . . . . . . . . . . . . . . . . 39

3.2.1 Continuous-Time Models . . . . . . . . . . . . . . . . . 39

3.2.2 Discrete-Time Models . . . . . . . . . . . . . . . . . . . . 42

3.3 Simulation Results . . . . . . . . . . . . . . . . . . . 43

3.3.1 Continuous-Time Models . . . . . . . . . . . . . . . . 44

3.3.2 Discrete-Time Models . . . . . . . . . . . . . . . . . . . . 51

3.4 Comparison of the First-Order and the Second-Order Models . . . . . . . 55

3.4.1 Comparison of the Time Constant and the Damping Ratio . . . . 55

3.4.2 Comparison of the Correlation Coefficients and Least Mean Square

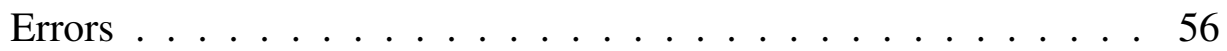

3.5 Summary $\ldots \ldots \ldots \ldots \ldots \ldots \ldots \ldots \ldots \ldots \ldots$

4 The Proposed Model for Coupling 59

4.1 Introduction . . . . . . . . . . . . . . . . 59

4.2 Coupling Identification $\ldots \ldots \ldots$

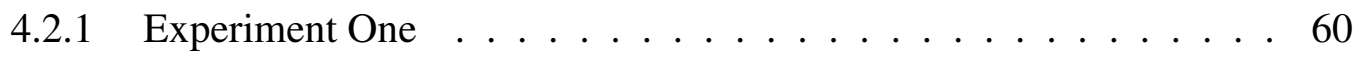

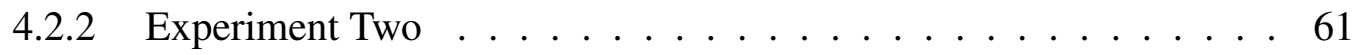

4.2 .3 Analysis . . . . . . . . . . . . . . 63

4.3 The Proposed Models _ . . . . . . . . . . . . . . . . 63 
4.3.1 Models of the Coupling . . . . . . . . . . . . . . . . . 64

4.3.2 Hybrid Learning Procedure . . . . . . . . . . . . . . . . . 64

4.3.3 ANFIS Architecture . . . . . . . . . . . . . 65

4.4 Simulation Results . . . . . . . . . . . . . . . . . . . . . . 67

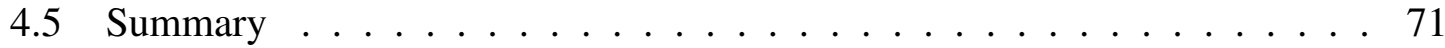

5 The Proposed Control Methods for a Meat Drying Room 72

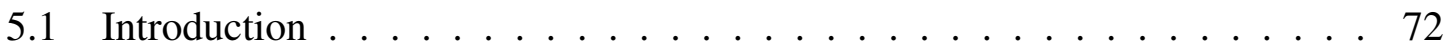

5.2 The Proposed Control Methods . . . . . . . . . . . . . . . . . . 73

5.2.1 Analog Controller Design _ . . . . . . . . . . . . . . 73

5.2.2 Digital Controller Design . . . . . . . . . . . . . . . . . . . . 74

5.2 .3 Fuzzy Controller Design . . . . . . . . . . . . . . 76

5.2.4 PI-like Fuzzy Logic Controller Design . . . . . . . . . . . . . . 77

5.3 Simulation Results . . . . . . . . . . . . . . . . . . . . . . . . . 79

5.3.1 Analog Control Systems _ . . . . . . . . . . . . . 80

5.3.2 Digital Control Systems _ . . . . . . . . . . . . . . 88

5.4 Comparison of the Control Methods _ . . . . . . . . . . . . 96

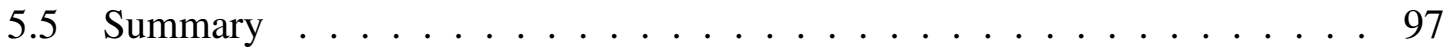

6 Conclusions and Future Works 99

6.1 Conclusions . . . . . . . . . . . . . . . . . . . . . . . . 99

6.2 Future Works . . . . . . . . . . . . . . . . . . 100

$\begin{array}{ll}\text { References } & 101\end{array}$

$\begin{array}{ll}\text { Appendix } & 109\end{array}$

A Relative Humidity Sensors 109

B Real Data of a Drying Room 113

$\begin{array}{lll}\text { C Membership Functions } & 116\end{array}$ 


\section{List of Figures}

2.1 Psychrometric chart . . . . . . . . . . . . . . . . . . . . . . . 14

2.2 Air-conditioning processes in a psychrometric chart $\ldots \ldots \ldots \ldots$

2.3 The RBF networks structure $\ldots \ldots \ldots \ldots \ldots \ldots$

3.1 The block diagram of drying room control systems $\ldots \ldots \ldots$

3.2 The step responses of temperature from a real meat drying room . . . . . 41

3.3 The step responses of relative humidity from a real meat drying room . . . 41

3.4 The best step responses of the first-order temperature model for a meat drying room $\ldots \ldots \ldots \ldots \ldots \ldots \ldots \ldots \ldots \ldots \ldots \ldots \ldots \ldots$

3.5 The step responses by changing $G_{t}$ in the first-order temperature model . . 45

3.6 The step responses by changing $\tau_{t}$ in the first-order temperature model . . . 46

3.7 The best step responses of the first-order RH model for a meat drying room 47

3.8 The step responses by changing $G_{h}$ in the first-order RH model . . . . . . 47

3.9 The step responses by changing $\tau_{h}$ in the first-order RH model $\ldots \ldots$. . . 48

3.10 The best step responses of the second-order temperature model for a meat drying room . . . . . . . . . . . . . . . . . . . . . . 49

3.11 The best step responses of the second-order relative humidity model for a meat drying room . . . . . . . . . . . . . . . . . . . . . 49

3.12 The best step responses of the discrete first-order temperature model for

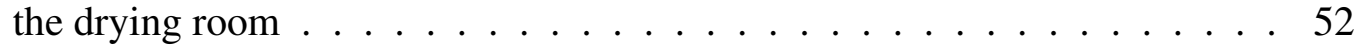

3.13 The step responses by changing $T_{s}$ in the discrete temperature model for the drying room $\ldots \ldots \ldots \ldots \ldots \ldots \ldots \ldots$ 
3.14 The best step responses of the discrete first-order relative humidity model for a meat drying room . . . . . . . . . . . . . . . . . 54

3.15 The step responses by changing $T_{s}$ in the discrete relative humidity model for a meat drying room . . . . . . . . . . . . . . . . 54

4.1 A coupling signal from the current meat drying room . . . . . . . . . 61

4.2 Input membership functions before the training of the ANFIS model . . . . 62

4.3 Input membership functions after the training of the ANFIS model . . . . . 62

4.4 The relationship between coupling temperature and coupling relative hu-

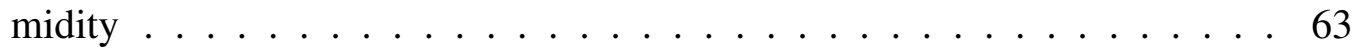

4.5 The network structure of ANFIS . . . . . . . . . . . . 65

4.6 The membership functions of input disturbance temperature before the training of ANFIS for a model of the coupling signal . . . . . . . . . 69

4.7 The ANFIS RMSE changing results. . . . . . . . . . . . . . . . . . . . 69

4.8 The ANFIS training results. . . . . . . . . . . . . . . . . . 69

4.9 The ANFIS testing results $\ldots \ldots \ldots \ldots \ldots$

4.10 The membership functions of input disturbance temperate after the training of the ANFIS for a model of the coupling signal . . . . . . . . . . . . 70

4.11 Comparison the simulation coupling signal with the real data from the meat drying room . . . . . . . . . . . . . . . . 71

5.1 The proposed fuzzy logic controller of relative humidity $\ldots$. . . . . . 77

5.2 The output of PID control without temperature disturbances . . . . . . 81

5.3 The output of PID control without relative humidity disturbances . . . . . . 81

5.4 The block diagram of PID control with disturbances . . . . . . . . . . . 82

5.5 The simulation results of PID control with disturbances for temperature . . 83

5.6 The simulation results of PID control with disturbances for relative humid-

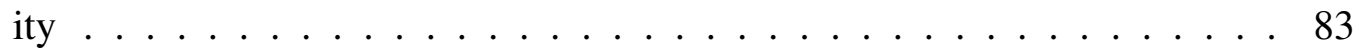

5.7 The input membership functions of the fuzzy controller . . . . . . . . 85

5.8 The outputs of membership functions of the fuzzy controller . . . . . 85

5.9 The block diagram of fuzzy PID control . . . . . . . . . . . . . 86 
5.10 The outputs of fuzzy PID control and PID control for RH . . . . . . . . 86

5.11 The block diagram of fuzzy control for relative humidity . . . . . . . . 87

5.12 The outputs of fuzzy control and PID control for relative humidity . . . . . 88

5.13 The block diagram of digital PI control for temperature and RH . . . . . . . 89

5.14 The output of digital PI control for temperature . . . . . . . . . . . . 90

5.15 The outputs of digital PI control for RH control . . . . . . . . . . . . 90

5.16 The block diagram of digital control for a meat drying room . . . . . . . 91

5.17 The flow diagram of the digital control systems . . . . . . . . . . 91

5.18 The temperature output of digital control for a drying room system . . . . . 94

5.19 The digital control of relative humidity for a drying room system . . . . . . 97

A.1 Capacitive RH sensors . . . . . . . . . . . . . . . . 110

A.2 Resistive RH sensors . . . . . . . . . . . . . . . . 110

A.3 Dew point hygrometers . . . . . . . . . . . . . . 111

A.4 The structure of a psychrometer . . . . . . . . . . . 111

A.5 An application of a hygrometer . . . . . . . . . . . . . . . . 112

C.1 Membership functions for input temperature errors . . . . . . . . . . 117

C.2 Membership functions for input temperature difference errors . . . . . . . . 117

C.3 Membership functions for temperature outputs . . . . . . . . . . . . . 117

C.4 Membership functions for input relative humidity errors . . . . . . . . . . 118

C.5 Membership functions for input relative humidity difference errors . . . . 118

C.6 Membership functions for relative humidity outputs . . . . . . . . . . 118 


\section{List of Tables}

3.1 The list of parameters for tuning a temperature transfer function. . . . . . 51

3.2 The list of parameters for tuning a relative humidity transfer function. . . . 55

3.3 Experimental and calctulated time constants and the damping ratios for temperature and relative humidity transfer functions. . . . . . . . . 56

3.4 The comparison of the correlation coefficients and the LMSE values of temperature transfer functions. . . . . . . . . . . . . 57

3.5 The comparison of the correlation coefficients and the LMSE values of relative humidity transfer functions. . . . . . . . . . . . . . 57

5.1 Fuzzy control rules of fuzzy PID control. NL: negative large; Z: zero; PL: positive large; NS: negative small; PS: positive small. . . . . . . . . . . . . 77

5.2 Fuzzy control rules of the temperature controller. NL: negative large; Z: zero; NS: negetive small; PS: positive small. . . . . . . . . . . . . . . 78

5.3 Fuzzy control rules of the relative humidity controller. NL: negative large; Z: zero; NS: negetive small; PS: positive small. . . . . . . . . . . . . . 79

5.4 Comparison of relative-humidity control performances. . . . . . . . . . . 97

B.1 The real data of temperature for a drying room system . . . . . . . . . . . 114

B.2 The real data of relative humidity for a drying room system. . . . . . . . 115 


\section{List of Symbols}

\begin{tabular}{|c|c|}
\hline$A, U$ & The fuzzy sets \\
\hline$A_{t}$ & The constant which becomes 0.5 at the temperature above $0^{\circ} \mathrm{C}$ \\
\hline$A_{1}, A_{2}, A_{3}$ & The fuzzy sets for ANFIS \\
\hline$a, u_{f}$ & The elements of fuzzy sets \\
\hline$a_{1}, b_{1}$ & The parameters of $z$ function \\
\hline$a_{1 h}, b_{1 h}$ & The parameters of relative humidity $z$ function \\
\hline$a_{1 t}, b_{1 t}$ & The parameters of temperature $z$ function \\
\hline$a_{t 1}, c_{t 1}$ & The premise or input parameters of fuzzy inference system \\
\hline$c_{i}$ & The centers of the basic functions \\
\hline$C_{t}$ & The temperature constant \\
\hline$d_{h}$ & The coupling of relative humidity for a digital control system \\
\hline$d_{t}$ & The coupling of temperature for a digital control system \\
\hline$E$ & The error of inputs of relative humidity control system \\
\hline$E_{h}$ & The error of relative humidity for the PID controller \\
\hline$E_{i h}$ & The overall error measurement of relative humidity for a fuzzy logic controller \\
\hline$E_{h p}$ & The $p$-th error measurement of relative humidity \\
\hline$E_{m s e}$ & The mean square error \\
\hline$E_{\text {oh }}$ & The error of outputs of relative humidity \\
\hline$E_{t}$ & The error of temperature for the PID controller \\
\hline$e_{i h}$ & The error of an input of relative humidity for a digital PI controller \\
\hline$e_{i t}$ & The error of an input of temperature for a digital PI controller \\
\hline$e_{o h}$ & The error of an output of relative humidity for a digital PI controller \\
\hline$e_{o t}$ & The error of an output of temperature for a digital PI controller \\
\hline
\end{tabular}




\begin{tabular}{|c|c|}
\hline$e_{r h}$ & The error of relative humidity control system \\
\hline$\Delta \mathrm{e}_{i h}$ & The difference error of an input of relative humidity for a digital PI controller \\
\hline$\Delta \mathrm{e}_{i t}$ & The difference error of an input of temperature for a digital PI controller \\
\hline$f_{h}()$ & The overall output functions of relative humidity of fuzzy inference \\
\hline$G$ & The gain of the system \\
\hline$G_{h}$ & The gain of relative hmidity control system \\
\hline$G_{t}$ & The gain of temperature control system \\
\hline$H()$ & The identify function of fuzzy inference system \\
\hline$h$ & The single layer of the hidden layer \\
\hline$H_{r c}$ & The output of relative humidity \\
\hline$H_{r d}$ & The disturbance relative humidity \\
\hline$H_{r s p}$ & The relative humidity set point \\
\hline$K_{p h}$ & The proportional gain of the PID controller for relative humidity \\
\hline$K_{i h}$ & The integral gain of PID controller for relative humidity \\
\hline$K_{d h}$ & The derivative gain of PID controller for relative humidity \\
\hline$K_{p t}$ & The proportional gain of the PID controller for temperature \\
\hline$K_{i t}$ & The integral gain of PID controller for temperature \\
\hline$K_{d t}$ & The derivative gain of PID controller for temperature \\
\hline$k$ & The sampling index \\
\hline$k_{f}$ & The fuzzy rule index \\
\hline$k_{h}$ & The index of sampling for relative humidity control \\
\hline$k_{t}$ & The index of sampling for temperature control \\
\hline$L$ & The total number of layers \\
\hline$M_{h}$ & The PID controller output for relative humidity \\
\hline$M_{t}$ & The PID controller output for temperature \\
\hline$m$ & The layer index \\
\hline$N$ & The net of neural networks \\
\hline$n$ & The total number of samples \\
\hline$n_{i}$ & The number of index \\
\hline$O_{h}$ & The actual output vector of relative humidity \\
\hline
\end{tabular}




$\begin{array}{ll}O_{h 1}^{4} & \text { The adaptive node and an output node function of relative humidity of layer } 4 \\ O_{h 1}^{5} & \text { The overall output of relative humidity of layer } 5 \\ O_{i} & \text { The objective values of temperature and relative humidity } \\ O_{t 1}^{1} & \text { The node of the layer } 1 \text { as a set of temperature data of the fuzzy membership function } \\ P & \text { The training data pairs } \\ p & \text { The element of training data } \\ p_{h}, r_{h} & \text { The consequent parameters } \\ Q_{t} & \text { The temperature ratio } \\ q^{-1} & \text { The delay operator } \\ R() & \text { The function of a defuzzification } \\ R_{h} & \text { The input of the relative humidity system } \\ R_{i} & \text { The factor of weights } \\ R_{s} & \text { The input of a dynamic system } \\ R_{t} & \text { The input of the temperature system } \\ r & \text { The correlation coeffcient } \\ r_{1 h}, r_{0 h} & \text { The control parameter of relative humidity for a digital PI controller } \\ r_{1 t}, r_{0 t} & \text { The control parameter of temperature for a digital PI controller } \\ S_{t} & \text { The set of total parameters } \\ S_{t 1} & \text { The set of premise parameters } \\ S_{t 2} & \text { The set of consequent parameters } \\ T & \text { The input temperature of the fuzzy model } \\ T_{c} & \text { The temperature control output } \\ T_{f}() & \text { The transfer function of a system } \\ T_{d} & \text { The transfer function of relative humidity system } \\ T_{f h}() & \text { The the of } p \text {-th target output vector of relative humidity } \\ T_{h} & \text { The relative humidity system } \\ T_{h t} & \text { The }\end{array}$




\begin{tabular}{|c|c|}
\hline$T_{s p}$ & The temperature set point \\
\hline$T_{t}()$ & The transfer function of the temperature system \\
\hline$T_{t d}$ & The disturbance temperature \\
\hline$T_{t d e}$ & The dead time of the temperature system \\
\hline$T_{t t}$ & The time of the temperature system \\
\hline$T_{w}$ & The wet bulb temperature \\
\hline$t$ & The time \\
\hline $\mathbf{u}$ & The vector of a control input signal \\
\hline$u$ & The input of a digital system \\
\hline$V_{h 63}$ & The value at $63 \%$ step responses for the relative humidity system \\
\hline$V_{h m}$ & The max value of step responses for the relative humidity system \\
\hline$V_{t 63}$ & The value at $63 \%$ step responses for the temperature system \\
\hline$V_{t m}$ & The max value of step responses for the temperature system \\
\hline$w$ & The weight \\
\hline $\bar{w}$ & The fixed weight \\
\hline $\mathrm{X}$ & The unknown vector of training data \\
\hline $\mathrm{x}$ & The vector of variables of control signal \\
\hline$x_{m}$ & The $m$-th multidimensional input vector \\
\hline$x_{f}, y_{f}$ & The variables of membership functions \\
\hline$x_{i}, y_{i}$ & The two values for calculating correlation coeffcient \\
\hline$x_{p}$ & The input variable of a perceptron model \\
\hline$x_{t}$ & The variable of a membership function \\
\hline$Y$ & The output of a perception model \\
\hline $\mathbf{y}$ & The vector of control output measurements \\
\hline$Y_{h}$ & The output of relative humidity control \\
\hline$Y_{s}$ & The output of a dynamic system \\
\hline$Y_{t}$ & The output of temperature control \\
\hline$y$ & The output of a digital system \\
\hline$y_{h}$ & The output of controlled relative humidity for a digital control system \\
\hline$y_{t}$ & The output of controlled temprature for a digital control system \\
\hline
\end{tabular}


$\eta_{h}$

$\mu$

$\omega_{n}$

$\sigma$

$\xi$

$\tau$

$\tau_{h}$

$\tau_{t}$

$\#$

The variable of the $Z$ function

The generic parameter

The learning rate

The membership function

The frequency of the second-order transfer function

The radii of a basis function

The damping ratio

The time constant

The time constant of relative hmidity

The time constant of temperature

The number of layers 


\section{List of Abbreviations}

$\begin{array}{ll}\text { AI } & \text { Artificial Intelligence } \\ \text { ANFIS } & \text { Adaptive-Network-Based Fuzzy Inference System } \\ \text { FIS } & \text { Fuzzy Inference System } \\ \text { FLC } & \text { Fuzzy Logic Controller } \\ \text { HVAC } & \text { Heating, Ventilation, and Air Conditioning } \\ \text { IIR } & \text { Infinite Impulse Response } \\ \text { LSE } & \text { Least Squares Estimate } \\ \text { LMSE } & \text { Least Mean Square Error } \\ \text { MF } & \text { Membership Function } \\ \text { MIMO } & \text { Multiple-Input and Multiple-Output } \\ \text { NIR } & \text { Near-Infrared Spectroscopy } \\ \text { NL } & \text { Negative large of the error of inputs } \\ \text { NS } & \text { Negative small of the error of outputs } \\ \text { PD } & \text { Proportional Derivative } \\ \text { PI } & \text { Proportional Integral } \\ \text { PID } & \text { Proportional Integral Derivative } \\ \text { PL } & \text { Positive large of the error of inputs } \\ \text { PS } & \text { Positive small of the error of outputs } \\ \text { PT } & \text { Radinum thermometers } \\ \text { RBF } & \text { RBF }\end{array}$


WNN

$\mathrm{Z}$

Wavele Based Artificial Neural Network

Zero error of fuzzy inputs 


\section{Chapter 1}

\section{Introduction}

A control system for a meat drying room consists of a temperature control system and a humidity control system, which can also be called a microclimate control system. In the research of temperature and humidity control systems, coupling between temperature and relative humidity is difficult to deal with due to humidity fluctuations. Since humidity variance is a complex and nonlinear process, humidity has only been partially studied for meat processing (Arnau et al., 2003). Temperature and humidity are two important factors in meat drying processing directly affecting meat product quality. Thus, temperature and relative humidity control are essential to meet standards of quality and safety requirements during meat drying processing.

To control temperature and relative humidity, the systems of heating, ventilating, humidifying and dehumidifying are standard equipment in a meat drying room, and the equipment is very expensive. Due to design requirements, models should be accurate to simulate a better process in terms of purchasing physical equipment. If designers use accurate models to simulate realistic control systems, projects can be designed and implemented in a timely and cost effective way.

The structure of a meat drying control system includes sensors, controllers, and actuators. Sensors for a meat drying control system measure temperature and humidity as input signals. A commonly used device for measurements of the temperature and humidity is called a psychrometer. This psychrometer consists of two temperature sensors; one is called dry-bulb; and another is called wet-bulb. The controlled actuators in the meat drying 
room are steam valves, and glycol valves provide the function of heating or cooling, and humidifying or dehumidifying.

In the structure described above, current control systems of a meat drying room are reliability, stability, and simplicity. However, a main drawback of control systems is a fluctuation of coupled signals between temperature and relative humidity. This coupling leads to fluctuations in the humidity control systems when temperature is changed in the system. Therefore, the relative humidity variable becomes more complex and nonlinear. This coupling issue raises the question about how humidity is controlled in an effective way and how it is monitored to meet quality and safety requirements during meat drying processing. This question drives scientists and engineers to study and develop intelligent control systems for meat drying control systems. Currently, commonly used control systems for a meat drying room in meat manufacturing control the temperature and humidity simultaneously, as two separate control loops (a temperature control loop and a humidity control loop). A PID control algorithm used in most applications in the industry. This method is for a linear control system. However, for complex and nonlinear systems, the PID control algorithm is not able to accurately control for temperature and humidity. Therefore, an advanced control algorithm is required for the humidity control loop. Energy savings are also a big concern for meat drying processes.

\subsection{Problem Statement}

Generally speaking, a fluctuation of coupled signals between temperature and relative humidity exists in any temperature and humidity control system such as HVAC, greenhouse, livestock, etc. Control systems like these can be divided into four parts: an input signal, a controller, an output signal of plant, and a measured feedback signal from the output. For the measured feedback signal, precise measurements are a challenge because they generate an error in a control system. For the controller, methods for decoupling the disturbance signal between the temperature and humidity are difficult. However, these factors directly affect precise performance of control systems and result in large energy consumption for meat drying systems. 
This research studies problems of coupling and proposes methods to solve the problems in control systems. The problems include accurate measurement of temperature and relative humidity, coupling signals between temperature and relative humidity, and accurate control of meat drying room systems, which will be stated in detail as follows:

1. Coupling between temperature and humidity in control systems

Coupling in process control systems in a drying room is problem that requires research. This problem is caused by temperature fluctuations that affect the relative humidity in the control system. If the temperature is controlled in a range of $\pm 0.1^{\circ} \mathrm{C}$, the coupling problem can be ignored (Ma et al., 2013). However, in practice, temperatures cannot be controlled that precisely, resulting in nonlinear coupling which adds complexity in controlling this system. A commonly used control method is PID control for the temperature and relative humidity simultaneously, using two closedloop control loops. This method has many advantages such as being simple to use with one sensor and one set of control hardware, however, this method cannot solve a complex and nonlinear problem, which is the coupling problem. In this case, researchers have developed decoupling methods to solve the problem to make better process control systems. This coupling problem is seen not only in meat drying room control systems, but also in many applications such as HVAC, livestock, greenhouse, etc., therefore, research into coupling is valuable to study in the future.

\section{Temperature and humidity control for meat drying room systems}

Coupling is present in current meat drying systems, which causes humidity fluctuations. An improvement in control accuracy for a meat drying room is not only to increase the quality of meat products, but also to reduce energy cost in temperature and relative humidity control systems. Normally, temperature and relative humidity control systems use steam, low temperature glycol, and water with air pressure as mediums to control temperature and relative humidity in a meat drying room (Ma et al., 2013). Both types of equipment ( steam generator systems and refrigeration systems) are high energy cost installations. If the control accuracy of temperature and relative humidity control system are low, the energy cost will be higher. 
Energy consumption in specific meat drying control systems is related to temperature and humidity control accuracy. If temperature and humidity control are more accurate, energy consumption will be less resulting in cost efficiency. Meanwhile, humidity must not negatively affect the quality and safety of meat products during meat drying processing. To control the humidity properly, questions are raised about how to select an instrument for temperature and humidity measurements, in order not to affect inputs of the control systems for sensing feedback signals. Methods for humidity control have to provide suitable ways for solving complex and nonlinear problems in disturbance conditions. For complex and nonlinear problems, advanced control methods will be involved to solve the problems.

In this thesis, measurement methods of temperature and relative humidity will be introduced by means of literature review. Control and a model of coupling will be proposed and simulated for a highly controlled performance of control systems in a meat drying system.

\subsection{Objectives of This Thesis}

The problems in control systems have been stated in detail as described earlier. In this thesis, the objectives of the research project are to propose and develop advanced control methods for solving the problems in control systems for a meat drying room. The aim is to develop a complete control system which will be simulated and tested in Matlab. The system of meat drying room will include plant models, a model of coupling, and advanced control methods for a control loop of relative humidity.

The objectives of the research project can be summarized as follows:

- To develop plant models for temperature and relative humidity. Real data will be taken from a current meat drying room system. Methods of simulation plant models for the system will be selected from mathematical models of the first-order model and the second-order model for conducting simulation experiments. The output of the simulation models will be compared with the real data to verify simulation results using Matlab in experiments. 
- To develop a model for a complex and nonlinear coupling. Methods of fuzzy and neural networks will be selected for the model to conduct simulation experiments. The learning algorithm of neural networks should be used in the model for experiments. Finally, the model of coupling will be provided into control systems of temperature and relative humidity to evaluate simulation results.

- To develop methods of control systems for a meat drying room. Methods of fuzzy PID control and PI-like fuzzy control will be selected for controlling the temperature and relative humidity to conduct simulation experiments. A complete model of control systems will be developed for control simulations of a meat drying room system. The fuzzy inputs will be set up according to real data from a meat drying room system. The complete control systems for a drying room will be evaluated in Matlab.

\subsection{Contributions of This Thesis}

This research project developed a complete control system for a drying meat room. Contributions can be summarized as follows:

- Equations of transfer functions were developed for modeling the temperature and relative humidity of a meat drying room. A method for obtaining these equations used real data of temperature and relative humidity. The models of transfer functions for a meat drying room can evaluate the systems of temperature and relative humidity. The models of output response can be compared with real data for temperature and relative humidity to verify the systems. Simulations of a first-order model and a second-order model were done by a control system tool in Matlab and were used to develop systems modeling obtained from transfer functions. The method of obtaining the equations adjusted to be nearest the graph of real data of temperature and relative humidity can be used in other applications. Meanwhile, the equations of transfer functions were applied into simulations of a complete control system for meat drying control systems.

- A model of coupling was developed for temperature and relative humidity control 
systems for a real meat drying room. Experiments were done for analyzing and identifying the coupling and proved that the coupling is complex and nonlinear. Due to the coupling being a complex and nonlinear, a method for developing the model used adaptive-network-based fuzzy inference system (ANFIS). This method is an advanced technology, which is very suitable for obtaining the model of the coupling. The model also was applied to control systems of temperature and relative humidity, and simulation results showed a good match with real control systems. In the literature review of this research project, there are no studies found on the model of coupling.

- A complete control system was developed for a meat drying room. This control system is the main contribution for this research project and is unique for a real meat drying. The intelligent control method was used. There are two approaches to develop the complete control system. One method is analog control, and the other is digital control including a fuzzy PID controller for relative humidity in a continuous-time model and a PI-like fuzzy logic controller in a discrete-time model for temperature and relative humidity. The two methods of control systems both performed much better. The results are demonstrated in this thesis.

\subsection{Organization of This Thesis}

This thesis is organized into six chapters to show the research project in a thesis manner. This chapter introduces an overview of the research project including problem statement, objective, contributions, and organizations.

Chapter 2 provides a technical background and overview for currently used instrumentation for temperature and relative humidity measurements, methods of systems modeling, control methods for temperature and humidity, methods for a model of disturbance signals, and methods of control systems for a meat drying room.

Chapter 3 presents proposed methods for systems modeling of a meat drying room. In this chapter, the block diagram of control systems shows the control flow in details such as inputs, controllers, plants, outputs, and feedback signals. The models have been designed 
with a continuous-time model and a discrete-time model for simulations. The section of simulations shows the equations of transfer functions and the results of systems modeling for temperature and relative humidity.

Chapter 4 includes a proposed method for modeling disturbance signals of coupling based on an ANFIS model in Matlab. The training data of temperature and relative humidity used real data. A hybrid learning algorithm was applied for training. The simulations have been done and are demonstrated the results.

Chapter 5 shows two complete methods of control systems for a meat drying room with two inputs, two outputs, two closed-loop feedback control loops, two disturbance signals, a fuzzy PID for humidity, and two PI-like fuzzy controllers for temperature and relative humidity. The simulation experiments used a fuzzy control tool in Matlab and demonstrate very good results.

Chapter 6 concludes features of the proposed complete control system for a meat drying control and indicates future works of this research project. 


\section{Chapter 2}

\section{Background and Literature Survey}

The research project is focused on systems modeling and intelligent control of meat drying processing in a meat drying room. Literature review is divided into five parts: the investigation of temperature and relative humidity measurements, systems modeling, coupling model, control methods of temperature and relative humidity, and energy concerns for meat drying systems.

\subsection{Temperature and Relative Humidity Measurements}

In the previous section, temperature and humidity present very important factors for meat drying processing in a meat drying room, however, humidity measurements are more difficult to obtain than other parameters, such as temperature measurements and velocity of air measurements (Lin and Chen, 2005). Meat drying processing has been studied by scientists. Temperature is a well-known factor for microbial stability (Leistner, 1986) and affects proteolysis and some sensorial properties (Arnau et al., 1997; Parolari et al., 1994); however, the effect of humidity has only been partially studied in meat processing (Arnau et al., 2003). Mannheim et al. (1994) indicated that humidity was a critical parameter affecting most food products. In this case, an investment in instruments for relative humidity measurements for studying the humidity control system more deeply has been made. 


\subsubsection{Measurement Methods}

There are many methods to measure relative humidity. Every method is required to meet the conditions of precision and accuracy, a response time, a range of ambient temperatures, a range of humidity of interest, types and amount of contaminants in the air, flow rate of the air, barometric pressure, maintainability of the apparatus, and cost (Oliver, 1971). One important requirement for control systems is a high precision instrument for sensing relative humidity. Problems can be caused by an inaccurate sensor, electrical signal acquisitions, or instrument calibration to the relative humidity measurement. These factors will affect the precision and accuracy in the measurement of the temperature and the relative humidity for drying room control systems. Therefore, to obtain a highly accurate and long-term working sensor for measuring relative humidity signal in harsh environments is a challenge.

Humidity is the amount of water vapor contained in the air and is expressed by three methods of humidity measurement: absolute humidity refers to the mass of the water vapor per-unit-volume of air; relative humidity refers to the quantity of water vapor present to the quantity of saturate of the air at the ambient air temperature, and expressed as a percentage; dew point is the air temperature at which condensation of water vapor occurs, and if this temperature is below the freezing point of water, then it is commonly known as the "frost point" (Fisher et al., 1981). Psychrometry utilizes the fact that a one-to-one relationship exists between the evaporation rate of water and the moisture content of the air at a given ambient air temperature, pressure, and fixed ventilation rate. The psychrometry method of measuring humidity involves a pair of values known as dry-bulb and wet-bulb temperatures (Fisher et al., 1981).

To monitor and control a meat drying room, instruments of relative humidity measurement have to be used. Mathioulakis et al. (2011) describes methods of humidity measurement. The measurement set-ups used for the quantity of humidity in the air can be classified in two main categories: direct measurement where the measurement setup is primarily a function of the same humidity quantity as the measurand of interest such as a capacitive relative humidity sensor and indirect measurement where the measurement setup responds to a primary humidity quantity different from the measurand of interest 
and conversion equations are needed for calculating the value of the measurand such as psychrometer (Mathioulakis et al., 2011).

The direct measurement method is simple and low cost; however, it is not appropriate for applications of high metrological performance. The low metrological performance of these devices is mainly related to the problematic behavior of the materials used as hysteresis phenomena, change in the behavior of porous or fibrous media due to potential material contamination, low reproducibility, etc. (Mathioulakis et al., 2011).

The indirect measurement method can be conditionally achieved through the implementation of higher metrological performance, potentially reaching that of a standard method (Mathioulakis et al., 2011). Disadvantages of this method are the relatively high cost and complicated use related to the devices of this category (Mathioulakis et al., 2011).

Each of the methods of humidity measurement has advantages and disadvantages with measurement techniques. The goals of the overall measurement determine the method for an application. Instruments of relative humidity measurement have direct humidity measurement and indirect humidity measurement. Instruments of direct humidity measurement are likely to be capacitive relative humidity sensors (Fig. A.1), resistive relative humidity sensors (Fig. A.2), or chilled mirror hygrometers in dew-point temperature measurements (Fig. A.3), etc. Instruments of indirect humidity measurement are likely to be a psychrometer (Fig. A.4), or a hygrometer (Fig. A.5), etc. These sensors can be used in a wide range of applications for relative humidity measurement and automatic control systems such as for the food industry, greenhouses, livestock, HAVC, chemical industry, etc.

A specific feature of psychrometer is adaptability. It can long term work in a harsh measurement environment than most relative humidity sensor such as capacitor humidity sensor and resistive humidity sensor. The psychrometer for relative humidity measurement will be reviewed in more detail in this thesis.

A psychrometer consists of a pair of dry-bulb temperature probe and wet-bulb temperature probe to measure the evaporation rate in the air (Fisher et al., 1981). Thus a matched pair of electrical thermometers, one maintained in a wetted condition, typifies an industrial psychrometer. Powell (1936) investigated a psychrometer for the purpose of determining the manner in which the wet-bulb depression depended on the degree of ventilation, the 
diameter of the wire, the thickness and length of the water film covering the junction of the wet thermocouple, and the relative positions of the wet and dry thermocouples. These factors could cause problems for the psychrometer. Powell (1936) introduced an example that a psychrometer constructed of thirty-three wet and dry copper-constant thermocouples as bulbs connected in series, as a means of observing from a distance the relatively small wet-bulb depressions obtained in cold-storage holds on board ship.

A digital ventilated psychrometer was studied by Nantou (1979), composed of an operational amplifier with the input circuit of a combined thermistor and two resistors. The experimental result showed that the experimental psychrometer could measure a range of $\mathrm{RH}$ from 20 to $100 \%$ with a temperature range from 0 to $50^{\circ} \mathrm{C}$ at an error of relative humidity of less than $2 \%$ and the response time in less than 1 min. From Nantou (1979)'s research, the material of dry-bulb and wet-bulb for measuring temperature had been developed using a type of resistor that is called a thermistor. This temperature sensor performed quite well for signal conditioning for data acquisition used in applications of a digital psychrometer and an automatic control system. The literature review of the psychrometer for relative humidity measurement has been done, but there are not many articles that talked about the history of psychrometer, most describe it.

Researchers study instruments of temperature and humidity measurement, especially humidity measurement because humidity measurement is more complicated in industrial environments. The instrument of humidity measurement has many applications such as the high humidity range validation on gelatine gel (Baucour and Daudin, 2000), drying of rough rice (Ondier et al., 2010), drying of paddy (Srzednicki et al., 2006), selected quality attributes of bread rolls (Schirmer et al., 2011), drying of chicken meat (Nathakaranakule et al., 2007), storage of dried garlic slices (Prachayawarakorn et al., 2004), bulk drying of chicory roots with respect to process (Hoang et al., 2004), invasive and noninvasive firmness measures (Hertog et al., 2004), etc. To meet requirements of temperature and humidity measurement, current instruments on the market have direct measurement and indirect measurement methods for relative humidity measurements. The follow section will introduce the instruments of temperature and relative humidity measurement in detail with emphasis on the psychrometer. 


\subsubsection{Psychrometers}

For relative humidity measurement, a psychrometer is used and constructed with a pair of wet-bulb and dry-bulb thermometers to measure the evaporation rate in the air. A matched pair of electrical thermometers, one maintained in a wetted condition, typifies an industrial psychrometer (Fisher et al., 1981). The dry-bulb temperature is the temperature of atmospheric air and the wet-bulb temperature is the adiabatic saturation temperature.

A psychrometer was investigated by Powell (1936) for the purpose of determining manners in which the wet-bulb depression depended on the degree of ventilation, the diameter of the wire, the thickness and length of the water film covering the junction of the wet thermocouple, and the relative positions of the wet and dry thermocouples. These factors can cause problems for the psychrometer.

Psychrometry is just a manner of relative humidity measurement. However, the structure of a psychrometer that utilizes psychrometry to construct itself is quite different.

An example of a psychrometer structure was introduced by Neiva et al. (2006). This structure was designed with an axial micro-fan to promote air motion through the psychrometer. Air speed in the wick area, closed to the throat, was measured with a hot-wire sensor. The air flow was horizontal, and an upper chamber was built to measure dry-bulb temperature. Therefore, any humidity from the wet wick would not interfere with the drybulb measurement. In the lower chamber, the wet (PT100) bulb was tied to a suspended cotton wick, which thoroughly covered the sensor bulb, and its opposite end was dipped in a water basin (Neiva et al., 2006).

Another example of a psychrometer was constructed from 40-gauge wires and consisted of wet and dry thermocouples differentially connected together and a separate dry thermocouple (Powell, 1936). As the psychrometer was mounted in a closed chamber, the two dry thermocouple junctions were in place where they were not influenced by the cooler wet thermocouple. In the chamber, the relative humidity of the air varied from about $20 \%$ to $100 \%$. Observation of the dry temperature and depression of the wet-bulb were recorded (Powell, 1936).

Comparing the two studies above, both had a structure with a fan, but the size of the 
fan and where to locate the fan were different. Both were structured with dry-bulb and wet-bulb sensors, but where the two sensors were located, how big the wet cotton wick and distance to the water basin were different. The psychrometer structure has an effect on precision and accuracy of relative humidity measurement.

\section{Methods to Obtain Values of Relative Humidity}

The ventilation psychrometer indirectly measured the relative humidity by reading the temperature on the wet and dry bulbs, and the relative humidity was based on measurements of the temperature ratio between the dry and wet bulbs and converted the temperature to a voltage (Nantou, 1979). Then the voltage was calculated using Sprung's formula to convert the dry and wet bulb temperature signals into a signal conditioning for relative humidity measurement. In this study a method of calculating relative humidity is introduced. Sprung's formula for an Assmann ventilation psychrometer is used to convert a signal of dry-bulb and wet-bulb temperatures into a signal of relative humidity (Nantou, 1979). The relation between the saturated vapor pressure and the dry-bulb temperature is nonlinear. Therefore, the relation between the relative humidity and the dry-bulb temperature also is nonlinear. The relation shows a strong effect of the temperature to the relative humidity in the humidity measurement, which can be called a coupling.

The temperature ratio $Q_{t}$ of the dry and wet bulbs for the ventilated psychrometer can be defined as (Nantou, 1979)

$$
Q_{t}=\frac{T_{w}-C_{t}}{T_{d}-C_{t}}
$$

where $C_{t}$ is a constant in degrees Celsius, $C_{t}=-16^{\circ} \mathrm{C}$. The relative humidity is a function of the temperature ratio $Q_{t}$ with a range of the temperature from 0 to $50^{\circ} \mathrm{C}$ and a range of the relative humidity from 20 to $100 \%$ with an error of less than $2 \%$ (Nantou, 1979).

Figure 2.1 is a chart for finding a relative humidity value at a specific temperature, and the chart is suitable for the dry-bulb temperature from $35^{\circ} \mathrm{F}$ to $120^{\circ} \mathrm{F}$ and wet-bulb/dewpoint temperature from $35^{\circ} \mathrm{F}$ to $85^{\circ} \mathrm{F}$. A range of the relative humidity value that is shown in the chart is from $10-100 \%$. The psychrometric chart has been used less often since the digital psychrometer invented. Fig. 2.2 shows air-conditioning processes in a psychrometric chart. 


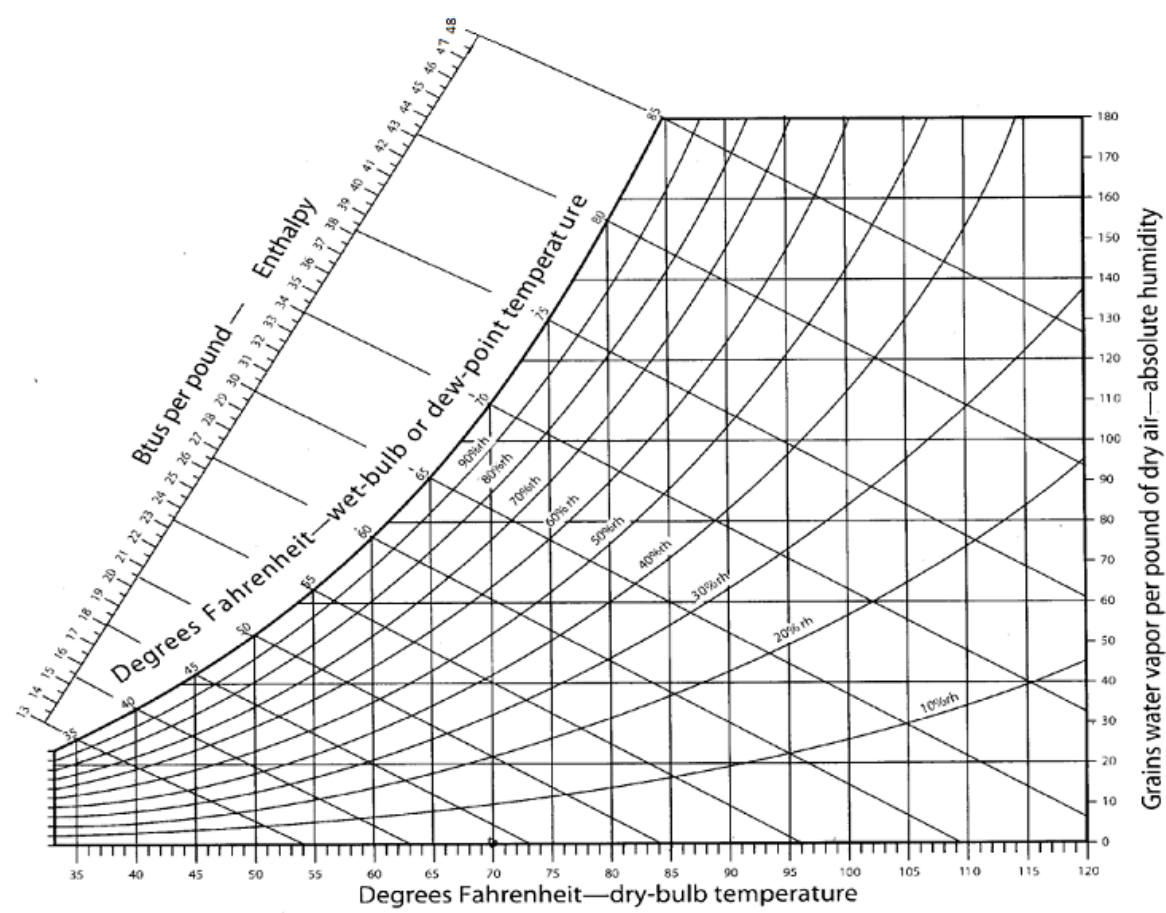

Figure 2.1: The psychrometric chart. (from: http://ngwindows.com/blog/tag/psychrometricchart/)

\section{Electrical Signal Conditioning}

Electronic psychrometer can be classified into analog psychrometer and digital psychrometer. An analog psychrometer generates a voltage of the analog output signal which has a one-to-one relationship to the moisture content of atmospheric air (Fisher et al., 1981). The analog signal of the psychrometer connects to a strip-chart recorder, a meter, and/or the input of an analog process controller. A digital psychrometer generates a voltage of digital output signal which is available in a form of coded binary words and drives a digital panel meter or a digital process controller (Fisher et al., 1981).

The electronic psychrometer is constructed with two thermistors, which are called a wet-bulb and a dry-bulb. The electronic psychrometer is interfaced directly to a microprocessor without the need of a separate A or D converter or other complex analog signal conditioning circuitry for relative humidity measurement. Fisher's study introduced two circuits along with digital signal processing algorithms. In the first method, the thermistors were built into a dual resistor-controlled-oscillator circuit. Oscillator frequencies were 


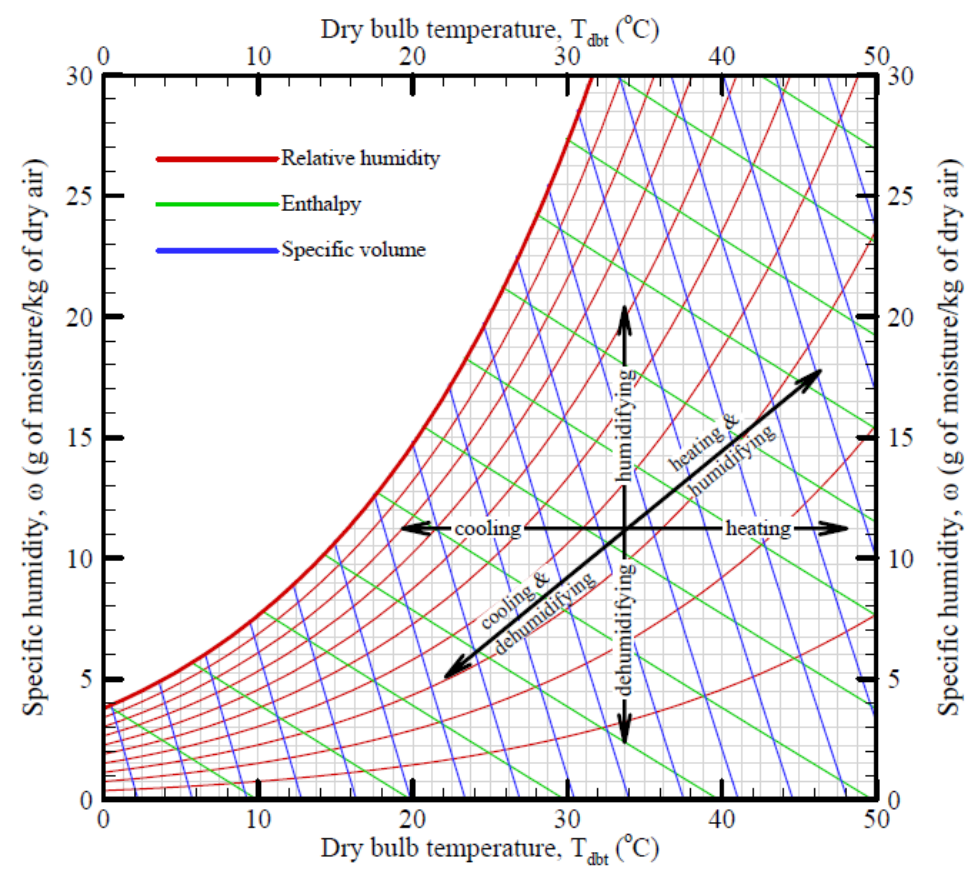

Figure 2.2: Air-conditioning processes in a psychrometric chart. (from: (Mahmud, 2013))

mapped into the temperatures of the dry-bulb and wet-bulb. In the second method, the thermistors resided in a pulse-width-modulation circuit. Pulse-widths were mapped into the temperatures of the dry-bulb and wet-bulb. Each circuit calculated the transfer function and sensitivity coefficients (Fisher et al., 1981).

As mentioned earlier, relative humidity is a function of a temperature ratio $Q$. The relationship between the relative humidity and the temperature ratio is shown in Fig. 2.1. Fisher et al. (1981) studied converting the temperature ratio to relative humidity. A voltage was set by a variable resistor of $1 \mathrm{k}$. The temperature ratio was calculated by circuits that had a math function. Output voltage of the circuits was proportional to the temperature ratio, and the circuits converted the output voltage to the relative humidity values.

\section{Calibration}

Calibration procedures were introduced by Neiva et al. (2006). The procedures were for the electronic psychrometer which: first calibrated temperature sensors, second determined a psychrometric coefficient, and third calculated humidity values. The result of accuracy obtained for the electronic psychrometer was $2.62 \% \mathrm{RH}$. 


\section{Advantages and Disadvantages}

Advantages of an electronic psychrometer described by Fisher et al. (1981) are: (1) widely used in many applications (2) simplicity (3) low cost (4) relative ease of maintenance (5) being most accurate near $100 \% \mathrm{RH}$ and (6) being superior in this region to virtually all other sensor types. The main important advantages of the electronic psychrometer are that it can be used above $100^{\circ} \mathrm{C}$ with the wet bulb up to the boiling point of water (Fisher et al., 1981).

Disadvantages of the electronic psychrometer are: (1) the temperature measurements are difficult to get if the temperature of the wet-bulb is lower than the freezing point of water; (2) a source of moisture is provided by the electronic psychrometer and can be intolerable under certain applications; (3) as relative humidity drops below $20 \% \mathrm{RH}$, cooling of the wet-bulb to its full depression becomes difficult, especially when the sensors are aspirated at an airstream rate of $10 \mathrm{~m} / \mathrm{s}$ (Fisher et al., 1981).

\subsection{Systems Modeling}

A math representation of physical systems is called a model, which is used to reason about the behavior of a system. In a dynamic system, actions do not occur immediately, and a behavior of a dynamic system evolves with time (Åström and Murray, 2008). Daskalov (1997) introduced a method of systems modeling for air temperature and humidity using dynamic discrete auto-regressive moving average models. The method of models was using first-order dynamic models. Sebastian et al. (2005) studied the heat and mass transfer modeling for meat drying and smoking. Feyissa et al. (2011) developed a coupled heat and mass transfer model with mathematical equations for a contact baking processing. Caponetto et al. (2000) used a method of soft computing for systems modeling of a greenhouse.

In general, there are two ways to view a model of a dynamic system: an internal view and an external view. The internal view, which comes from classical mechanics, presents the internal workings of a dynamic system (Åström and Murray, 2008). Models built on the internal view can be given names such as internal description, state model or white box model. The external view, which is from both input and output, presents the outside 
workings of the dynamic systems. Models built on external view can be given names such as external description, input/output or black box model (Åström and Murray, 2008).

A model of a control system aims to precisely represent a dynamic system such that it meets a control objective. The model is selected depending on the phenomena of interests in the control system. Commonly the methods of models used in feedback and control systems are differential equations and difference equations (Åström and Murray, 2008).

\subsubsection{Continuous-Time Models}

Mathematical models of systems use math equations to perform functions of a system. Commonly used math models for control are ordinary differential equation.

To model the effects of external disturbances and control forces of the dynamic system, the ordinary differential equation is used (Åström and Murray, 2008). Many electrical engineering systems can be modeled by linear and time-invariant systems; also there are many ways to describe models of electrical engineering system performance such as a step response of linear system, and a frequency response of linear system (Åström and Murray, 2008).

When the ordinary differential equation is applied to a control system, the system model has to take into consideration the external influences such as external control forces and sensors. Therefore, the model equation is given as (Åström and Murray, 2008)

$$
\begin{gathered}
\frac{d \mathbf{x}}{d t}=\mathbf{f}(\mathbf{x}, \mathbf{u}), \\
\mathbf{y}=\mathbf{g}(\mathbf{x}, \mathbf{u}),
\end{gathered}
$$

where $\mathbf{u}$ is a vector of control input signal, $\mathbf{x}$ is a vector of variables, and $\mathbf{y}$ is a vector of control output measurements. If $\mathbf{u}$ has been properly selected, a variable $x$ will reach controllability. If enough information is included in the measurement to reconstruct the state, the measurements are observability. A set of variables $\left[x_{1}(t), x_{2}(t), \cdots, x_{n}(t)\right]$ in the dynamic system presents the state of a system and determines the future behavior of the

dynamic system (Dorf and Bishop, 2008). As noted, the input or output model is not only for the class of linear and time-invariant systems, but also is suitable for nonlinear systems. 
A transfer function for systems modeling is commonly used. The transfer function of a system is a ratio that is an output variable over an input variable. The transfer function is an external view of dynamic systems, which represents and describes the input-output of the behavior of a system. The transfer function $T_{f}$ of a first-order system is given as (Landau and Zito, 2006)

$$
T_{f}(s)=\frac{G}{\tau s+1},
$$

where $\tau$ is the time constant of the system, $G$ is the gain of the system. The transfer function of a second-order system is given as (Dorf and Bishop, 2008)

$$
T_{f}(s)=\frac{\omega_{n}^{2}}{s^{2}+2 \xi \omega_{n} s+\omega_{n}^{2}},
$$

where $\omega_{n}$ is the natural frequency, and $\xi$ is the damping ratio.

\subsubsection{Discrete-Time Models}

Discrete-time modeling is used in a digital control algorithm. Landau and Zito (2006) introduced the discrete-time model. An input $u(t)$ and a output $y(t)$, which are sampled using a sampling period, are obtained as number sequences in a time $t$ or a sampling index $k$. The time is normalized discrete-time, which is the real time divided by the sampling period, $t=\frac{t}{T_{s}}$, where $T_{s}$ is a sampling time period. This is a time domain, and the equation of the discrete-time model is given as

$$
y(t)=-a_{1} y(t-1)+b_{1} u(t-1)
$$

where $a_{1}$ and $b_{1}$ are parameters for adjusting the output $y(t)$.

With more consideration for the discrete-time model, the delay operator $q^{-1}$ is used, and Equation ( 2.6) is rewritten as (Landau and Zito, 2006)

$$
y(t-1)=q^{-1} y(t)
$$

and

$$
\left(1+a_{1} q^{-1}\right) y(t)=b_{1} q^{-1} u(t)
$$


The discrete-time model can be performed by the discretized differential equations of the continuous-time model. With the normalized time, the equation can be written as (Landau and Zito, 2006)

$$
\frac{d y}{d t}=-\frac{1}{\tau} y(t)+\frac{G}{\tau} u(t)
$$

which can be rewritten in a discrete-time model as (Landau and Zito, 2006)

$$
y(t+1)+\left(\frac{T_{s}}{\tau}-1\right) y(t)=\frac{G}{\tau} T_{s} u(t)
$$

where $a_{1}=\frac{T_{s}}{\tau}-1, a_{1}<0$, and $T_{s}<\tau$; and $b_{1}=\frac{G}{\tau} T_{s}$.

The transfer function of a first-order system is given by (Landau and Zito, 2006)

$$
T(s)=\frac{b_{1} e^{-s T_{s}}}{1+a_{1} e^{-s T_{s}}}
$$

Letting $z=e^{s T_{s}}$, the transfer function of a first-order in $z$-transform is given as (Landau and Zito, 2006)

$$
T\left(z^{-1}\right)=\frac{b_{1} z^{-1}}{1+a_{1} z^{-1}},
$$

where $z$ is a variable of the $z$-transform function.

\subsubsection{Systems Modeling from Experimental Data}

Mathematical modeling is commonly used for systems modeling. A developed mathematical model was introduced by Mahajan et al. (2008) to understand the evolution of water loss with temperature and relative humidity for monitoring the mass loss of fresh mushrooms.

A method for systems modeling is "modeling from experiments" (Åström and Murray, 2008). This method is: first, obtain data from a physical dynamic system by conducting experiments; second, select a math equation for modeling a system; third, complete a simulation of the model; and fourth, compare the data of the simulation results with the data of the physical dynamic system in order to evaluate the selected math equation as a match for the systems modeling.

The method of "modeling from experiments" also can be applied to a feedback control system (Åström and Murray, 2008). A step unit signal is applied to an input; a step response of output signals is obtained when the system is steady-state; a math equation of transfer 
functions of the system can be determined using a graph of the step response of system outputs, and the parameters are identified the system by a time constant, a time delay, and a gain. The method of "modeling from experiments" also can identify a nonlinear system. To work on a complex system, a method of schematics diagram or block diagram for a system is useful for dealing with systems modeling. The method can represent a physical

system's essential features and hide irrelevant details (Åström and Murray, 2008). The schematics diagram uses simplified symbols with descriptions to draw the physical system, which identifies and builds a model of the system. In applications of control engineering, a block diagram has been used for representing a control system, and this is useful to build a model of a dynamic system. The block diagram is a graphic method that shows the information flow of the system using a block box to hide details of the system; each box comes with an input that goes into the box, and an output that leaves the box (Åström and Murray, 2008).

\subsection{Coupling Models}

The coupling of temperature and relative humidity was discussed by Becker et al. (1994). Liu and Da (2011) designed a method to solve the coupled relation between temperature and relative humidity. Zhang and Yang (2010) tracked relative humidity due to effects of temperature. The main drawback of coupling in a meat drying control system is to influence the accuracy of the relative humidity. To have a high accuracy of relative humidity, proper methods for solving the issue of coupling have to be considered for the control system of a meat drying room. Therefore, to develop a complete control system for a meat drying system, a coupling signal is needed. The model of the coupling will be applied to a control system simulation.

Adaptive-Network-Based Fuzzy Inference System has been discovered and selected because the coupling signal is complex and nonlinear. The ANFIS method based on the theory of fuzzy logic and neural networks is suitable for a complex and nonlinear problem. ANFIS method has been used in many applications. For example, Talei et al. (2010) studied rainfall-runoff modeling based on ANFIS; Riverol and Sanctis (2009) used ANFIS to 
improve the quality and minimize the fluctuation level of signals. In this section, ANFIS will be introduced in fuzzy logic and neural networks.

\subsubsection{Fuzzy Logic Based Models}

Advantages of fuzzy logic are that it is very simple to operate, very easy to implement programming, does not depend on precise math models, and is good for problems in uncertainty and adaptively. Fuzzy logic comes close to human thinking and can be thought of as a conditioned formalism of human thinking (Siddique and Adeli, 2013). Therefore, fuzzy logic has become more popular in an intelligent control system. Next, the theory of fuzzy logic will be introduced.

\section{Fuzzy Logic}

Fuzzy logic is based on fuzzy sets and logic theory to make decisions as humans thinking for controlling objects. In an intelligent control system, a fuzzy logic control plays an important role in adapting objects that are complex, nonlinear, uncertain, and unclear, and fuzzy logic control constrains objects based on rules (Siddique and Adeli, 2013).

One method that creates the most useful fuzzy rules is the "IF-THEN" rule statement to make decisions (Siddique and Adeli, 2013). This method uses fuzzy sets operations to be subjects and verbs of fuzzy logic; and includes rule forms, compound rules and aggregation of rules. For an example,

$$
\text { IF }<\text { fuzzy - proposition }>\text { THEN }<\text { fuzzy - proposition }>\text {. }
$$

In the process of a fuzzy operation, fuzzification is the first step to process a numeric value and converts it into a fuzzy input as a variable of membership functions; defuzzification is the last step and converts an output of fuzzy quantity into a clear value of object function.

\section{Fuzzy Inference}

Fuzzy inference is the mid step to process formulating, which is a nonlinear mapping from a given input space to output space (Siddique and Adeli, 2013); three inferences are as 
follows.

1. Mamdani fuzzy inference

A Mamdani-Type fuzzy inference uses a set of proposed rules to generate a single output that determines crisp values. Two types of fuzzy conditional statement rules as examples are given as (Shi and Hao, 2008)

(a) "IF THEN" rule type: IF $a$ is $A$ THEN $u_{f}$ is $U$, where $a$ is an element of $A$ fuzzy set, and $u_{f}$ is an element of $U$ fuzzy set

(b) Fuzzy implication rule type: Mamdani algorithm of fuzzy implication relations is given as

Fuzzy implication relation: $A(a) \rightarrow U\left(u_{f}\right)$

Defuzzification:

$$
R\left(a, u_{f}\right)=(A \rightarrow U)\left(a, u_{f}\right)=\min \left(A(a), U\left(u_{f}\right)\right)=A(a) \bigwedge U\left(u_{f}\right)
$$

where $R\left(a, u_{f}\right)$ is a function of defuzzification.

The inputs of a Mamdani algorithm can be one or two membership functions, but the output is one function; a Mamdani algorithm can be applied to any condition of fuzzy rules.

2. Sugno fuzzy inference

Sugno-Type fuzzy inference uses a systematic method to generate fuzzy rules; SugnoType fuzzy rules are created from an input $x_{f}$ or output $z_{d}$ data set that is given by a system. The defuzzification $z_{d}=f\left(x_{f}\right)$ is a function of crisp function (Siddique and Adeli, 2013).

If $x_{f}$ is $A$ then $z_{d}=f\left(x_{f}\right)$.

The inputs of Sugno-type fuzzy inference can be one as $x_{f}$ or two as $x_{f}$ and $y_{f}$ membership functions, but the output of the function $z_{d}=f\left(x_{f}, y_{f}\right)$ is only one. The rules can be $k_{f}=1,2,3, \cdots, n$. 


\section{T-S fuzzy inference}

T-S fuzzy inference of output functions is a linear function of inputs $x_{f 1}$ and $x_{f 2}$. The two types of output functions of T-S fuzzy inference are: 0 order if $x_{f 1}$ is $A_{1}$ and $x_{f 2}$ is $A_{2}$ then $z_{d}=k_{f}$, and 1 order if $x_{f 1}$ is $A_{1}$ and $x_{f 2}$ is $A_{2}$ then $z_{d}=p x_{f 1}+q x_{f 2}+r$, $A_{1}$ and $A_{2}$ are fuzzy sets. Where $p, q, r, k_{f}$ are constants.

There are two methods for calculating the system output (Shi and Hao, 2008). If there are $n$ rules, the $i$ is $i=(1,2,3, \cdots, \mathrm{n}), n \geq m$

(a) Sum weights

$$
Z_{w}=\sum_{i=0}^{m} w_{i} z_{d i}=w_{1} z_{d 1}+w_{2} z_{d 2}+\cdots+w_{n} z_{d m},
$$

where $w_{i}$ is $i$-th rule, $m$ is the position of weights.

(b) Average weights

$$
Z_{w}=\frac{\sum_{i=0}^{m} w_{i} z_{d i}}{\sum_{i=0}^{m} w_{i}}=\frac{w_{1} z_{d 1}+w_{2} z_{d 2}+\cdots+w_{n} z_{d m}}{w_{1}+w_{2}+\cdots+w_{m}} .
$$

There are two methods for calculating the weights.

(i) Minimum

$$
w_{i}=\min \left(\min \left(R_{i}, A_{1}^{i}\left(x_{f 1}\right)\right), A_{2}^{i}\left(x_{f 2}\right)\right) .
$$

(ii) Multiplication

$$
w_{i}=R_{i} A_{1}^{i}\left(x_{f 1}\right) A_{2}^{i}\left(x_{f 2}\right),
$$

where $R_{i}$ is the weight, usually $R_{i}=1$.

\subsubsection{Neural Network Based Models}

As the section discussed earlier, the functions of the fuzzy logic and membership functions are used for making decisions by following rules. Another technique is a learning capability like simulating human brain to do things in an intelligent system, which is called artificial neuron model. The artificial neuron model tries to build a model like human's learning. The human brain receives inputs from other sources by a biological neuron and executes a general nonlinear operation and outputs the final results (Siddique and Adeli, 2013). 
Math models of a neuron model have been used in an artificial neuron model; the weight vector $w$ has been considered for the strength of the connection between any two neurons. An output $Y$ of perceptron model includes inputs $x_{p i}$ as $i=1,2, \cdots, n$, weight $w_{i}$, net as $N$, and a nonlinear activation function, and the $N$ expresses as (Siddique and Adeli, 2013)

$$
N=\left(w_{1} x_{p 1}+w_{2} x_{p 2}+\cdots+w_{n} x_{p n}\right)
$$

and an output $Y$ of perceptron model is given as

$$
Y=f(N)
$$

where $f(N)$ is an output function.

The output function $f$ of RBF model is given as

$$
f(x)=\sum_{i=1}^{n} w_{i} R_{i}\left(x, c_{i}\right),
$$

where $w_{i}(i=1,2, \cdots, n)$ are weights, and $R_{i}\left(x, c_{i}\right)$ is the radial basis function. The RBF networks structure is shown in Fig. 2.3.

In the perceptron model, the activation function is nonlinear, and activation functions commonly used are step function, linear function, ramp function, and sigmoid function.

Neural network architectures can be divided into two catalogues: feedforward network and recurrent network ( feedback network) (Siddique and Adeli, 2013).

Feedforword networks include multilayer perceptron networks, radial basis function networks, generalized regression neural networks, probabilistic neural networks, belief networks, hamming networks, and stochastic networks.

Due to the coupling existing in a meat drying room, the system is a nonlinear problem. Therefore, methods for a nonlinear problem were investigated and focused on in this research project. The Radial Basis Function (RBF) network is a suitable function for a nonlinear problem, and the RBF network consists of a single layer and hybrid learning procedure, therefore, the RBF networks will be introduced below.

Radial basis function networks have receptive field units, which can also be named hidden units, and an activation function of the $i$ th receptive field unit is given as (Siddique and Adeli, 2013)

$$
h_{i}(x)=R_{i}\left(\frac{\left\|x-c_{i}\right\|}{\sigma_{i}}\right) \text {, }
$$




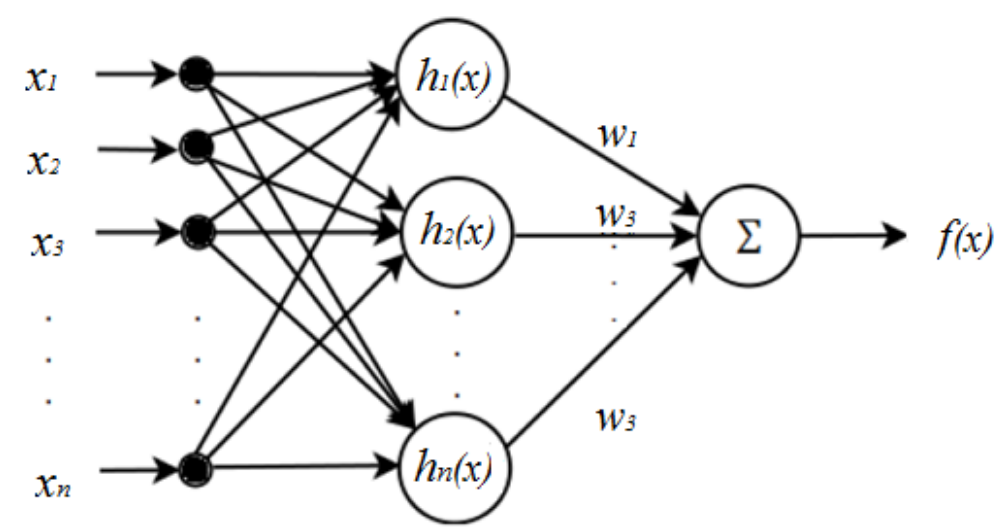

Figure 2.3: The RBF networks structure. (redraw from: (Siddique and Adeli, 2013))

where $R_{i}(\cdot)$ denotes the $i$-th radial basis function of the activation function, which has a single maximum at the center; $\|\cdot\|$ denotes the value is absolute value; $m$ th multidimensional input vector is denoted by $x_{m}$; the centers of the basic functions is denoted by the $c_{i}$; and the radii of a basis function is denoted by $\sigma_{i}$. The basis function $R_{i}(\cdot)$ can be defined as a Gaussian function (Haykin, 2009)

$$
R_{i}(x)=\exp \left(-\frac{\left\|x-c_{i}\right\|^{2}}{2 \sigma_{i}^{2}}\right) .
$$

An advantage of the radial basis function network is no connection between weights of the input layer and the hidden layer (Siddique and Adeli, 2013). Because of RBF networks advantage, it has many applications in any sort of model, linear or nonlinear functions, any sort of network, and single-layer or multi-layer. If parameters of a basis function are moved to the center and radius, the RBF network is nonlinear.

A main characteristic of the neural network is a learning ability, and methods for the learning ability can be classified into two categories (supervised learning and unsupervised learning).

A method for a supervised learning, which moves the network outputs near to a target output, consists of an external learning signal, weights, biases of the network, and adaptive learning rule during learning process (Siddique and Adeli, 2013). This method for supervised learning minimizes errors of all learning process elements. Rule algorithms of the supervised learning use backpropagation learning algorithm, gradient descent, Delta rules, etc. The rules also provide a set of input or output training data for a proper network behaviour. Siddique and Adeli (2013) mentioned, the gradient descent learning algorithms 
and backpropagation are the most popular algorithms for a multilayered neural network. Therefore, learning rule algorithms of the gradient descent and backpropagation will be introduced in Chapter 4.

\subsubsection{Adaptive-Network-Based Fuzzy Inference Systems}

The advantages of ANFIS described in Jang (1993)'s article were: 1) used a hybrid learning procedure to refine fuzzy rules into a complex system; 2) trained the fuzzy rules by using the desired data sets; and 3) provided many mathematical models to choose from because a high flexibility of adaptive networks .

Algorithms of the supervised learning include a gradient descent rule and a backpropagation learning algorithm. The gradient descent rule is a variation of Hebb's rule to modify the delta error before applying the weights of input connections to reduce a difference between a desired output and an actual output of the network. This rule has an additional proportional constant and is tied into a learning rate for a final modified factor to apply into the weights. An algorithm of backpropagation learning is basically a gradient descent algorithm, and its learning procedure presents a set of pairs that are input and output patterns to find the minima errors. The backpropagation learning also extends the delta learning rule with more layers and calculates the weights and bias changes (Siddique and Adeli, 2013).

ANFIS architecture was introduced by Jang (1993), which is hybrid learning algorithm and fuzzy inference systems. The study includes two types of fuzzy reasoning, which are Takagi and Sugenos type. The ANFIS architecture has five layers in a forward network.

The model of coupling is based on learning paradigms for adaptive networks batch (offline) learning in ANFIS. A hybrid learning procedure of batch learning paradigm in ANFIS combines the gradient method and the least squares estimate (LSE) to identify parameters. A forward pass and a backward pass consisted of each epoch used in hybrid learning procedure of ANFIS. The consequent parameters were identified by the least squares estimate for the coupling model. The backward pass of the hybrid learning algorithm in the coupling model was applied to the error rate's backward propagation. The premise parameters could be updated by gradient descent. In the model of the coupling, one input is temperature, and 
one output is relative humidity. There were three rules made for fuzzy inference systems.

The hybrid learning algorithm in ANFIS combined the gradient method and the least squares estimate method for updating the parameters in the adaptive network (Jang, 1993). A forward pass and a backward pass consisted of each epoch used in the hybrid learning procedure. Furthermore, the forward pass of the hybrid learning algorithm was applied to the function signals to go forward until layer 4 . The consequent parameters were identified by the least squares estimate. The backward pass of the hybrid learning algorithm was applied to the error rate's backward propagation. The premise parameters could be updated by gradient descent. The overall output functions can be written as linear combinations of the consequent parameters, and the given values are premise parameters.

Adaptive-Network-Based Fuzzy Inference System was developed by Jang (1993), and he published an article of ANFIS in 1993. His study built models for nonlinear functions and identified nonlinear components in control fields; his study presented application examples of ANFIS. ANFIS can also be used for building models of nonlinear functions and an input-output mapping, which is based on actual input-output data pairs and human knowledge. The learning rule of ANFIS involves a hybrid learning procedure. The study discusses the comparison of artificial neutral networks with a fuzzy model. Application examples are included in the study and show strong evidence for applications of the ANFIS.

The theory of ANFIS shows that it can serve as a basis for constructing a set of fuzzy "IF-THEN" rules with appropriate membership functions and generating the stipulated input-output pairs (Jang, 1993).

Most fuzzy inference systems were classified into three types of fuzzy reasoning which are named as type 1, type 2 and type 3 . The type 3 (Takagi and Sugenos type) of fuzzy reasoning is used by ANFIS for generating fuzzy "IF-THEN" rules (Jang, 1993).

The operating process of ANFIS are first, in the fuzzification process, input variables in membership functions on the premise part are compared to get the membership values of each linguistic label; second, membership values on the premise part are combined to get the firing strength of each rule for generating the qualified consequents of each rule depending on the firing strength; finally, the qualified consequents are aggregated to produce the output. In the last step, a linear equation of input variables plus a constant term are 
combined for an output of each rule; therefore, the output is the weighted average of each rule output (Jang, 1993).

Basically, an adaptive network takes advantage of a multilayer feedforward network method. Each node in the multilayer feedforward network presents a particular function on incoming signals, and a set of parameters relates to this node. In adaptive network operating process, parameter sets of an adaptive network are divided into groups of parameter sets for each adaptive node to achieve a desired input-output mapping that updates parameters according to given training data and a gradient-based learning procedure (Jang, 1993).

An adaptive network was shown by Jang (1993) who was working in a multilayer network to calculate actual output vectors; and the adaptive network was produced by input vectors of the given training data.

\subsection{Control Methods for Temperature and Relative Hu- midity}

The purpose of meat drying room control systems is to control the temperature and relative humidity of drying air. For example, the effects of the parameters of temperature, relative humidity, air velocity, and turbulence of air can occur at any time during the long process of drying meat (Stawczyk et al., 2004). To control the temperature, relative humidity, air velocity, and turbulence of the air, equipment for heating, ventilating, cooling, humidifying and dehumidifying are included in a meat drying room such as a circulated fan, a dry fog generator as a system humidifier, a steam generator, and a refrigeration system. Also a psychrometer is used as an input instrument for simultaneous control of temperature and relative humidity. According to the characteristics of the psychrometer that were discussed earlier, the psychrometer matches the requirements of a current control system for a meat drying room. This research will use a psychrometer to simultaneously control temperature and relative humidity because temperature and humidity are critical control parameters in the meat drying process. The current control system of a meat drying room is a multivariable control system which has two input variables and two output variables. 


\subsubsection{Temperature and Relative Humidity Control}

The literature on simultaneous control of temperature and relative humidity has been reviewed. This control method is not only used in the meat drying process, but can also be applied in many applications such as those for refrigeration, HVAC, greenhouse, livestock housing, etc. Much literature shows the use of decoupling control methods to solve a coupling problem in a simultaneous control system for temperature and relative humidity. Methods of decoupling control can be divided into categories such as non-interacting control, fuzzy control, inherently robust adaptive nonlinear proportional integral control, robust control, the WNN-IIR algorithm, a $2 \times 2$ controller, and two PID controllers. The studies of each category will be described in detail below.

Techniques were introduced by Nakanishi et al. (1973) for designing a decoupling or non-interacting control system with simultaneous control of temperature and humidity in a confined space. The decoupling or diagonalization method was used for control and reduced the multivariable control system into two non-interacting control loops, with the Ziegler-Nichols method set up for each non-interacting control loop (e.g. (Nakanishi et al., 1973; Wang et al., 2008)). After development of this control method, studies were conducted with other applications such as HVAC systems etc. (Rentel-Gómez and VélezReyes, 2001).

A fuzzy control method was introduced by Becker et al. (1994) for a coupling issue of temperature and relative humidity. This method was rule-based for control strategies for refrigeration systems. The fuzzy logic accounted for the complex coupling of the dynamic variables of temperature and relative humidity in a straightforward method. In this study, using a fuzzy controller to decouple the temperature and relative humidity in refrigeration systems shows better simulation results. The control of thermodynamic coupling of temperature on relative humidity was designed by using the temperature error as an additional input for the fuzzy humidity controller. Today the fuzzy logic method has been used in many applications for controlling uncertain conditions.

A nonlinear control method was described by Daskalov et al. (2006) for creating an inherently robust adaptive nonlinear proportional integral control law for simultaneous con- 
trol of temperature and humidity in livestock buildings; this method was able to track varying set-points of temperature and humidity. The advantages of the technique are that it includes the capability of decoupling the manipulated variables and provides (1) an easy way to simultaneously control temperature and humidity without resorting to conflicts; (2) the ability to modify the set-point of each variable without affecting the other variables during operations; (3) fast and accurate tracking of varying set-points; and (4) fast disturbance attenuation, sufficient robustness, and small deviation errors under system parameter variations and unknown system disturbances.

A climate control for a greenhouse with variable speed fans and a variable-pressure fogging system was studied by Linker et al. (2011). The robust control, which guaranteed adequate performance of the controlled system, despite large uncertainties and disturbances was based on quantitative feedback theory. A partial decoupling method was applied to a greenhouse climate control system. Parameters of air enthalpy and humidity ratio were developed and used in the partial decoupling method for the greenhouse control system. A difference of this study from others was that it used ventilating for reaching a desired air enthalpy and fogging for reaching a desired humidity ratio (Linker et al., 2011).

A better control method was shown by Jahedi and Ardehali (2012) that could lower energy demand and consumption for heating, ventilating, and air conditioning (HVAC) systems. HAVC system is the same as the system of meat drying room most. In this study, the control method of a wavelet-based artificial neural network (WNN) combined a proportional derivative (PD) controller with self-tuning that had been developed and simulated, and a decoupled bi-linear HVAC system that used the variable air volume and the variable water flow for controlling the temperature and relative humidity in a thermal zone. Also, the HVAC system was nonlinear and controlled variables of temperature and relative humidity were coupled. The method of a WNN can be applied in series with an infinite impulse response (IIR) filter giving advantages in faster and more accurate identification of system dynamics which are needed for training batch mode on-line use and off-line. Therefore, the two algorithms combine into one that is called the WNN-IIR algorithm, and this was applied in self-tuning of two PD controllers for temperature and relative humidity. The control method using the WNN-IIR algorithm gave an enhanced efficiency of the 
HVAC system, which was achieved substantially lower consumption of energy during a heating transient operation (Jahedi and Ardehali, 2012). Soyguder and Alli (2009a) designed an expert system using ANFIS and optimization with fuzzy modeling approach for the temperature and humidity control in HVAC systems.

A classical and popular method was developed by Bi et al. (2000) for approximately decoupling a system via a $2 \times 2$ controller, with two PID controllers at diagonal positions. This was a multivariable process, and if an independent control loop was not adopted with a coupling issue, the resultant performance of control was usually poor. Therefore, the decoupling control for temperature and relative humidity is very important for an accurate and precise control system.

\subsubsection{Analog Control Systems}

Analog control system uses a method of a continuous-time model. A PID control algorithm in a continuous-time model is most popular used in industry for control systems. To design a control system, a mathematical model of a system has to be obtained; then a control algorithm has to be selected. Therefore, a control method is an important part for control systems.

To obtain a good control system, a PID controller has to be tuned. There are many ways to tune a PID controller and obtain a proper output. A commonly used tuning method is the Zieglel and Nichols method. They conducted a sample experiment with extract features, and experiments of a dynamic process for obtaining experiment data, and determined controlled parameters from the extract features (Åström and Murray, 2008). A manual method for tuning the PID control system is also used.

\subsubsection{Digital Control Systems}

Digital control system offers advantages as follows (Landau and Zito, 2006): 1) discretized plant models can be a direct design of tailored control algorithms; 2) control algorithms can perform better computing complex and nonlinear algorithms by increasing a sampling period; and 3) discrete-time dynamic models make use of various high performing control 
strategies.

A digital PI controller based on an analog PI controller transformed into a discrete equation is given as (Landau and Zito, 2006)

$$
u(t)=\frac{G\left(1-q^{-1}\right)+\frac{G T_{s}}{T}}{1-q^{-1}}[r(t)-y(t)],
$$

where $r(t)$ is a desired function, $q^{-1}$ is a delay operator.

An equation of a PI control signal is given as

$$
u(t)=u(t-1)-r_{0} y(t)-r_{1} y(t-1)+r_{0} r(t)+r_{1} r(t-1)
$$

where $r_{0}$ and $r_{1}$ are parameters.

The parameters of a transfer function in a continuous-time model are related to the parameters of a discrete-time model for PI controller. The parameters $r_{0}$ and $r_{1}$ in Equation (2.24) are given as

$$
\begin{gathered}
r_{0}=G\left(1+\frac{T_{s}}{T}\right), \\
r_{1}=-G .
\end{gathered}
$$

These equations give the relation between a continuous-time PI controller and a discretetime PI controller. If one is designed, the other can also be designed by using these equations.

To obtain models of systems, the stability of discrete-time systems can be described by a parameter $z$. In general, a discrete-time system is asymptotically stable if all the roots of the transfer function denominator are inside of the unit circle and the equation is given as (Landau and Zito, 2006)

$$
1+a_{1} z^{-1}+\cdots+a_{n} z^{-n}=0,|z|<1
$$

otherwise, if $|z|>1$, the discrete-time system is unstable.

\subsubsection{Fuzzy Logic Control for Temperature and Relative Humidity}

To obtain high quality of meat products, precise control methods for temperature, humidity and velocity of the air are required. In nature, meat drying is a long process. A manual 
control for months period is very inconvenient. In a traditional way of producing meat products, an expert readjusted the set points of relative humidity in staggered form manually. The disadvantages of a manual control system are the discontinuity of the product evaluation and cost of the expert's training (Stawczyk et al., 2004). Therefore, many research projects create and study process control systems of meat drying rooms, and researchers look for advanced methods for controlling a non-linear and multivariable system.

The fuzzy system is the same as a general system in that it describes functions of systems. A difference between the fuzzy system and the general system is that fuzzy system uses a descriptive language based on predicates of fuzzy logic, and the general system uses mathematical equations. The fuzzy system can handle nonlinearity and unknown or partially known systems (Siddique and Adeli, 2013). There are two identifications for fuzzy modeling; structure identification and parameter identification. Structure identification can be used to find input or output relations for forming the "IF-THEN" rules and parameter identification can be used to find a better performance for an objective function.

Fuzzy systems have inputs and outputs, which are presented by fuzzy variables and fuzzy relations. Fuzzy systems can justify complications or complexity and requires supervising with significant unmodeled and unanticipated changes in the controlled systems (Siddique and Adeli, 2013). A fuzzy logic controller consists of fuzzification, inference (rule based), and defuzzification. Also the fuzzy logic controller can be combined with a PID controller, which is called a fuzzy PID controller. When inputs of the observed data have been made ready for the fuzzification step, the next step is inference. The inference is formulating a nonlinear mapping from a given input space to an output space and involves all membership functions, fuzzy logic operations, and "IF-THEN" rules. The inference involves "IF-THEN" rules that are an important step to transfer human knowledge to fuzzy logic control. Defuzzyification connects to the output space, which is a mapping from a fuzzy control action into crisp control action. Mathematical defuzzification can be a mapping from a fuzzy vector $\mu$ with $n$ fuzzy sets to a real number.

A math model for a meat drying system should contain good estimates of drying rate, coefficients of heat and mass transfer, material residence time, etc.. The real process is characterized by complex dynamics; varying parameters in the meat drying process are full 
of uncertainties due to the complexity of transfer law and these parameters also related to the air of temperature and relative humidity during the meat drying process. Because of the complex dynamics and parameter uncertainties, monitoring the meat drying process is not a simple task and varies in time. A fuzzy control system was used, and the fuzzy logic method was ideal for controlling complex and nonlinear systems since it systematically handles ambiguity. This study also includes a remote control over a wide area network to monitor and control the meat drying process system (Stawczyk et al., 2004).

Two fuzzy control systems were developed by Gou et al. (2005) for a drying process of fermented sausages in an industrial dryer at a constant temperature. The on-line instrument measurements in two fuzzy control systems were related to expert evaluation for input variables (Stawczyk et al., 2004). The study challenged traditional systems of meat drying, which used artificial systems where recycling, relative humidity, and temperature of drying air were controlled by a heat pump. The artificial systems were used in two different fuzzy control systems that created different input variables, and consequently different fuzzy rules (Gou et al., 2005).

An on-line instrument measurement of parameters was introduced by Stawczyk et al. (2009) that related to the expert evaluation, which would be useful for improving a control system to avoid crust formation (Gou et al., 2005; Munoz et al., 2007). The crust formation was related to water content and water activity values. Due to a determination that water activity was sensitive to variations of air temperature and relative humidity, a filter function was needed in control. For on-line product evaluation, one of the most promising technologies was near-infrared spectroscopy (NIR), which had a rapid, simple, and simultaneous on-line determination for meat drying properties on the surface such as moisture content and water activity (Stawczyk et al., 2009). Soyguder et al. (2009) designed a self-tuning PID-type fuzzy adaptive control for an expert HVAC system.

The literature review for this research project did not find any precise math models for a nonlinear control system for a meat drying room, therefore, to solve a nonlinear issue of coupling between temperature and relative humidity, a fuzzy control method is good choice and suitable for the complex and nonlinear problems.

Most classical control system designs assume a controlled system is linear (Siddique 
and Adeli, 2013). In some cases, the controlled system is nonlinear. However, a fuzzy control method is suitable for complex and non-linear systems. Human knowledge can be applied in the fuzzy control that generates control rules for controlling complex and nonlinear systems. Many applications of the fuzzy control method have been applied to a variety of fields in industry today.

This research project summarizes many applications using fuzzy logic control, such as greenhouse environmental control (Pasgianos et al., 2003), applications in simultaneous control of both temperature and humidity (Wang et al., 2006), intelligent humidity control systems based on field-bus (Zhang et al., 2004), hybrid fuzzy-PID control technology (Liu and Xu, 2008), nonlinear HVAC systems (Chiang and Fu, 2006), inverted pendulum (Arabinda, 2008), wood drying kiln (Hu et al., 2009), timber drying (Zhang et al., 2003) intelligent dry-bulb and wet-bulb humidity sensor (Cai et al., 2011), etc. Only a few articles were found that talk about control systems of meat drying process.

The literature review for control methods in this thesis can be summarized into six methods. More of the literature is about simultaneous control for temperature and relative humidity using the decoupling control method. Another method in the literature was about a management and optimization system to link a control system of temperature and relative humidity (Bon et al., 2005). However, only the methods of typical control which may be relevant to this research project were selected to be introduced in this thesis.

\subsection{Energy Concerns for Meat Drying Systems}

Energy concerns involve many fields in industry, especially in the food industry. "Energy Efficiency and Management in Food Processing Facilities" (Wang et al., 2009) studies energy efficiency methods. Li et al. (2012) studied building energy-saving equipment control and developed a new control method using a variable air volume (VAV) air conditioning self-adaption to decouple temperature and humidity. Li et al. (2012) also introduced a fuzzy PID control and a RBF neural network decoupling control for reducing disturbances of coupling of temperature and humidity in control systems. Wang et al. (2009) developed a genetic optimization algorithm used in a PID decoupling control for energy-saving 
in VAV Air-Conditioning systems. Argüello-Serrano and Vélez-Reyes (1999) presented a nonlinear controller for a VAV heating, ventilation, and air-conditioning (HVAC) systems.

A high accuracy control was identified by Jahedi and Ardehali (2012) that could lower energy demand and consumption in heating, ventilating and air conditioning systems. Moreover, using an artificial intelligence (AI) based control algorithm could lower fluctuations for the temperature and relative humidity, which reduced energy demand and consumption for HVAC systems (Nassif et al., 2005). This study also indicates different parameters such as temperature and relative humidity that have more direct influence on the HVAC systems performance as well as nearly all applications (Chi et al., 2006; Salazar et al., 2007). Bi et al. (2000) showed a poorly tuned control system may waste energy and reduce occupant comfort, and it causes excessive and unnecessary wear of actuators.

\subsection{Summary}

In this chapter, literature for this research project has been reviewed and classified into five categories as instruments of temperature and relative humidity measurement, systems modeling, coupling models, control methods of temperature and relative humidity, and eneryg concerns. As a part of the background this section introduced theory and advanced methods. Many papers were searched, and suitable papers were selected and introduced in this thesis. The literature review also found that the simultaneous control method for temperature and relative humidity is useful in many applications in various fields. 


\section{Chapter 3}

\section{The Proposed Models for Meat Drying}

\section{Process}

Humidity is an important control parameter in meat drying processing, because it directly affects the quality and quantity of final meat products, and energy consumption. Humidity also plays an important role in other manufacturing operations such as printing factories, electronics shops, plastics and textile manufacturers, agricultural green houses, and curing facilities. Because coupling between temperature and humidity exists in control systems, humidity control becomes more complex and nonlinear. Since the research project of humidity control has wide applications in the industry, researchers have studied humidity control for a long time. This chapter will propose methods for obtaining models of temperature and humidity and use methods of a continuous-time model and a discrete-time model for meat drying room control systems that were introduced in the literature review and background section.

\subsection{Introduction}

In general, a meat drying room control, also called a climate control, regulates temperature and relative humidity. In climate control systems, energy cost is a major concern. To design meat drying room systems, models have to be used in a process of simulation. In the literature review section, no mathematical models were discovered for meat drying 


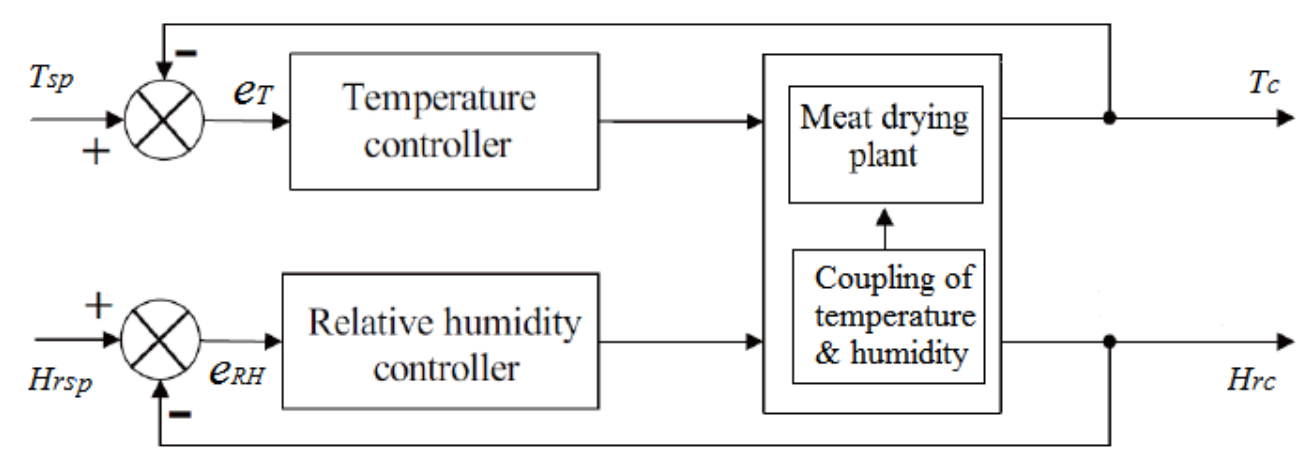

Figure 3.1: The block diagram of current drying room control systems.

room systems. However, if a climate system model had closely followed real systems, the climate system design would be simulated more realistically. If proposed models are accurate to the system, projects will save costs of equipment and time in the design process.

A drying room control system in meat manufacturing includes two distinct closed-loop control loops (a temperature control loop and a relative humidity control loop) as shown in Fig. 3.1, and controlled parameters are tuned independently. However, humidity control in a meat drying room is not an independent closed-loop control system since coupling between temperature and relative humidity is found and results in fluctuations of relative humidity control. The coupling drives more complications for measuring and sensing elements for humidity control systems.

In this research, a psychrometer was used for measuring and sensing temperature and relative humidity in meat drying control systems. A psychrometric law was followed by measurement elements of control systems. Therefore, the control systems were designed to have two inputs and outputs, called multivariable in and multivariable out (MIMO) as shown in Fig. 3.1. Fig. 3.1 also shows the meat drying systems include parts of a meat drying plant, a coupling effect of temperature and relative humidity, a PID controller of temperature, a PID controller of relative humidity, and two distinct closed-loop control loops (a temperature control loop and a relative humidity control loop). $T_{s p}$ is the temperature set point, $H_{r s p}$ is the relative humidity set point, $T_{c}$ is the output of temperature control, $H_{r c}$ is the output of relative humidity control, $e_{t}$ is the error of temperature, and $e_{R H}$ is the error of relative humidity.

As discussed earlier, the coupling in a meat drying room system results in inaccuracy 
control due to input fluctuation in relative humidity systems and unmodeled dynamic systems. A best model of a control system should work to change for inaccuracy control, which achieves a desired control objective and controls accurate performance.

\subsection{The Proposed Models}

A purpose of systems modeling is to obtain a precise representation model for a dynamic system, which achieves a control objective. Commonly used mathematical models in feedback control systems are differential equations and difference equations. In general, the commonly used a first-order system in a continuous-time model for obtaining transfer functions which is the Laplace transform equation. A second-order system for a closed-loop feedback control system to a step unit input is also used for systems modeling.

The step response of a system can be used to identify the system and determines parameters of rise time, peak time, percent overshoot and settling time. By knowing these

parameters, the system performance can be evaluated as either a steady-state system or a fluctuating system.

Equations of transfer functions can be used for a linear and constant parameter system. The mathematical models will be developed as models for obtaining transfer functions for a real meat drying room.

The proposed method of models for a meat drying plant was based an experimental data method. With this method the data were obtained by individual measurement values of temperature or measurement values of relative humidity from a real meat drying room, in order to build models for temperature and relative humidity systems. The data also were step response outputs of temperature and relative humidity systems.

\subsubsection{Continuous-Time Models}

The first approach for obtaining transfer functions for a meat drying plant is designed to use a first-order system and a second-order system in a time domain of continuous-time models. Experiments were to tune parameters in a model to match experimental data obtained from 
the real system as closely as possible. The real outputs of a meat drying system are shown in Figs. 3.2 and 3.3.

The first-order system of a linear model was used to obtain transfer functions of temperature and relative humidity for a meat drying room. The equation of a transfer function of the temperature system is given as

$$
T_{t}(s)=\frac{G_{t}}{\tau_{t} s+1},
$$

where $T_{t}(s)$ is a transfer function of temperature. To obtain the transfer functions of temperature, the parameters, $\tau_{t}$ and $G_{t}$ are tuned for models of the first-order system to closely follow the experimental data as shown in Fig. 3.2. The equation of a transfer function of the relative humidity system is given as

$$
T_{f h}(s)=\frac{G_{h}}{\tau_{h} s+1},
$$

where $T_{f h}(s)$ is the transfer function of relative humidity. To obtain the transfer function of relative humidity, the parameters, $\tau_{h}$ and $G_{h}$ are tuned for models of the first-order system to closely follow the real experimental data as shown in Fig. 3.3.

\section{Second-Order Systems}

A second-order system of a linear model was used to obtain transfer function of temperature and relative humidity, and the time constant $\tau$ and the gain $G$ for the initial values had been calculated for transfer functions. The parameters in the second - order system of linear Laplace formula was tuned repeatedly to obtain controllable systems, and a controllable system condition is defined as poles placed on the left hand side of the $s$ plane. The simulations were done for a meat drying plant in Matlab. Simulation graphs of temperature and relative humidity were matched with real control system graphs of temperature and relative humidity as shown in Figs. 3.2 and 3.3.

For simulation experiments, final equations of transfer functions were obtained for temperature and relative humidity systems. The models of temperature and relative humidity systems have been obtained using a first-order and second-order of linear Laplace methods. Therefore, an analysis of controllability is essential. Using a pole placement method analyzes the transfer functions of temperature and relative humidity as follows. 


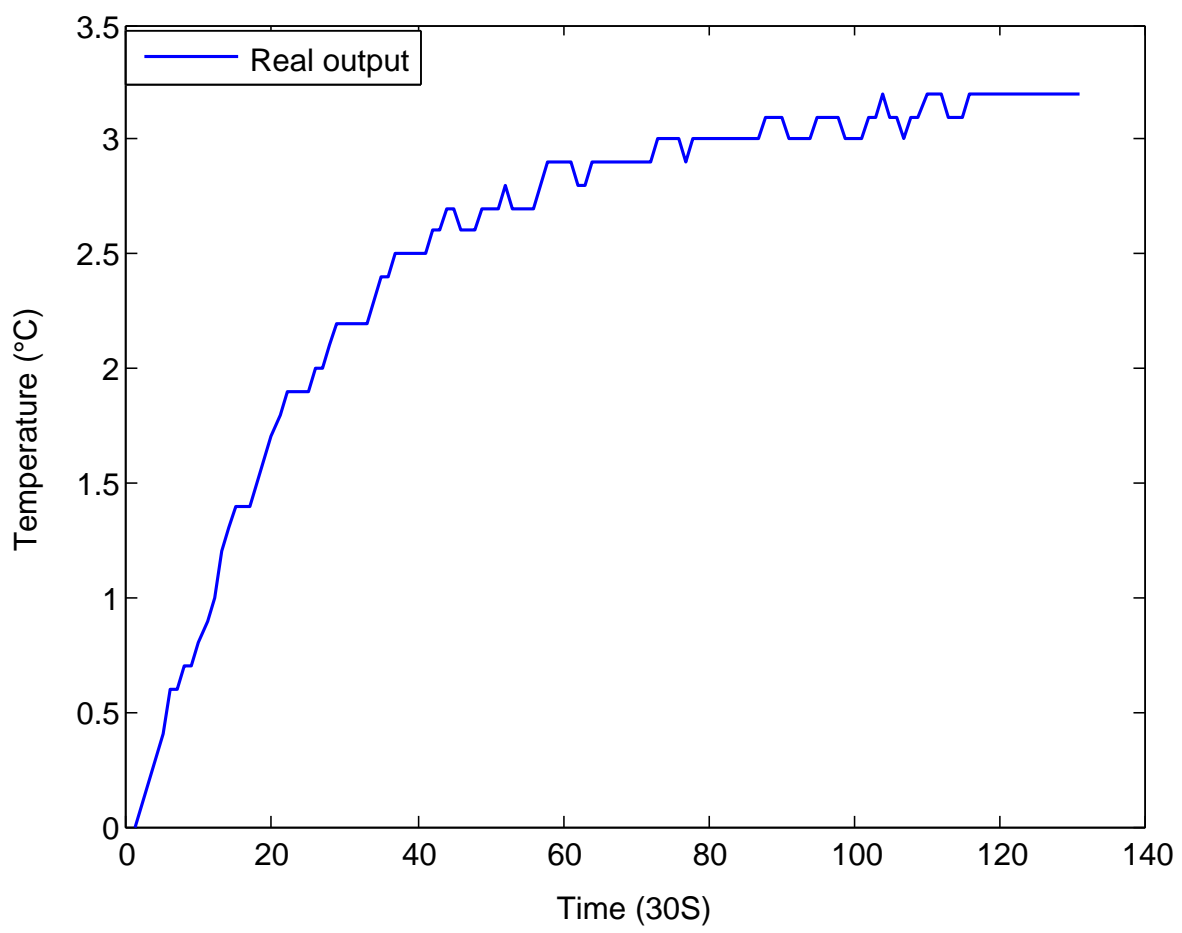

Figure 3.2: The step responses of temperature from a real meat drying room.

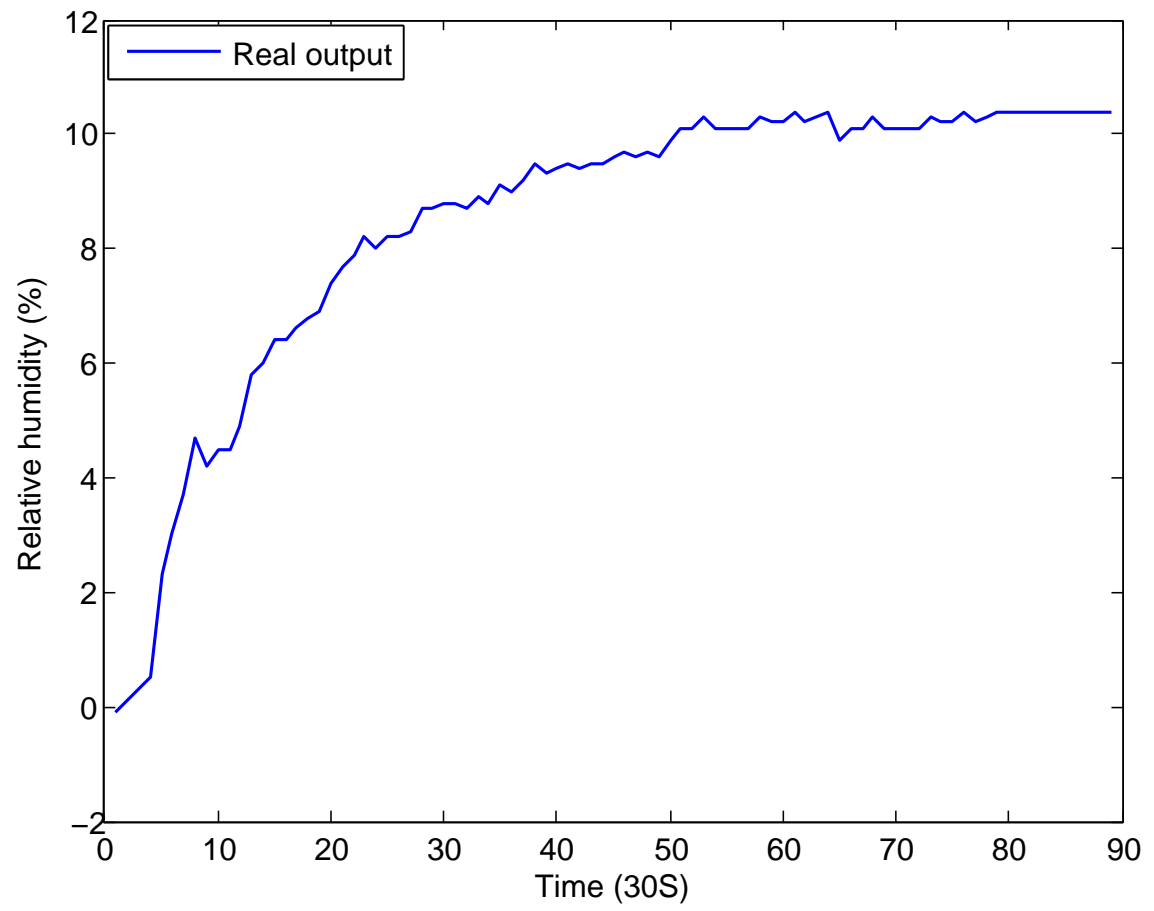

Figure 3.3: Step responses of relative humidity from a real meat drying room. 
1. The transfer function for the temperature is given as

$$
T_{t}(s)=\frac{Y_{t}(s)}{R_{t}(s)}=\frac{\omega_{n t}^{2}}{s^{2}+2 \xi_{t} \omega_{n t} s+\omega_{n t}^{2}},
$$

where $\omega_{n t}$ is the natural frequency of the temperature system, $\xi_{t}$ is the damping ratio, $Y_{t}(s)$ is the output of the temperature system, and $R_{t}(s)$ is the input of the temperature system.

2. The transfer function for the relative humidity is given as

$$
T_{f h}(s)=\frac{Y_{h}(s)}{R_{h}(s)}=\frac{\omega_{n h}^{2}}{s^{2}+2 \xi_{h} \omega_{n h} s+\omega_{n h}^{2}},
$$

where $\omega_{n h}$ is the natural frequency of the relative humidity system, $\xi_{h}$ is the damping ratio, $Y_{h}(s)$ is the output of the relative humidity system, and $R_{h}(s)$ is the input of the relative humidity system.

3. The poles of the transfer function Equation (3.3) of the temperature are calculated by

$$
s^{2}+2 \xi_{t} \omega_{n t} s+\omega_{n t}^{2}=0
$$

As a condition, if the poles lie on left side of $s$ plane, the system is controllable.

4. The poles of the transfer function Equation (3.4) of relative humidity are calculated by

$$
s^{2}+2 \xi_{h} \omega_{n h} s+\omega_{n h}^{2}=0 .
$$

As a condition, if the poles lie on left side of $s$ plane, the system is controllable.

\subsubsection{Discrete-Time Models}

The second approach for obtaining transfer functions of a meat drying plant was designed to use a discrete-time model in a time domain with a first-order system. Experiments were to tune parameters in a model of transfer functions to match real outputs of a meat drying plant show in Figs. 3.2 and 3.3 as closely as possible. 
In a first-order system of a discrete-time model used for obtaining transfer functions of temperature and relative humidity, the equation of a transfer function of temperature system is given as

$$
T_{t}(z)=\frac{b_{1 t}}{z+a_{1 t}}
$$

where $T_{t}(z)$ is the transfer function of temperature. To obtain the equation of transfer functions of temperature, the parameters, $a_{1 t}$ and $b_{1 t}$ are tuned for models of the first-order system to match as closely as possible the real experimental data in Fig.3.2. The parameters $a_{1 t}$ and $b_{1 t}$ were calculated for the model of the temperature system. Equations are given as

$$
\begin{gathered}
a_{1 t}=\frac{T_{s}}{\tau_{t}}-1, \\
b_{1 t}=\frac{G_{t}}{\tau_{t}} T_{s},
\end{gathered}
$$

where $T_{s}<\tau_{t}$, and $a_{1 t}<0$.

The equation of a transfer function of relative humidity system is given as

$$
T_{f h}(z)=\frac{b_{1 h}}{z+a_{1 h}}
$$

where $T_{f h}(z)$ is the transfer function of relative humidity. To obtain the equation of a transfer function of relative humidity, the parameters $\left(a_{1 h}\right.$ and $\left.b_{1 h}\right)$ are tuned for models of the first-order system to match as closely as possible the real experimental data in Fig.3.3. The parameters $a_{1 h}$ and $b_{1 h}$ were calculated for the model of the relative humidity system. Equations are given as

$$
\begin{aligned}
a_{1 h} & =\frac{T_{s}}{\tau_{h}}-1, \\
b_{1 h} & =\frac{G_{h}}{\tau_{h}} T_{s},
\end{aligned}
$$

where $T_{s}<\tau_{h}$, and $a_{1 h}<0$.

\subsection{Simulation Results}

Transfer functions of a meat drying plant were designed to use two model systems; a firstorder system and a second-order system for simulation experiments. The simulation experiments had been done for systems of a meat drying plant in Matlab. Experiments will 
identify which model is the best fit for meat drying systems. The systems identification and results discussion will be in the next section.

\subsubsection{Continuous-Time Models}

1. First-order system experiments:

Equation (3.1) is the transfer function for the temperature system, which was used for tuning a simulation model to obtain the best matched transfer function of temperature. Output of a simulation was graphed in a figure to match the output of a real output by changing a parameter, $\tau_{t}$ and a parameter, $G_{t}$. After tuning the first-order model, the best parameters of $\tau_{t}$ and $G_{t}$ are $\tau_{t}=24$ and $G_{t}=1$ and the transfer function model is given as

$$
T_{t}(s)=\frac{1}{24 s+1}
$$

The result of the best first-order temperature model for a meat drying room is shown in Fig. 3.4. Two tests were done for the temperature system. The first test tuned the parameter, $G_{t}$ in Equation (3.1) and assumed $\tau_{t}=24$. The test was done by changing $G_{t}$ as $G_{t}=0.5,1,1.5,2$. The result is shown in Fig. 3.5. The second test tuned the parameter, $\tau_{t}$ in Equation (3.1) and assumed the $G_{t}=1$ is the best value that was obtained from the first test. This test was done by changing $\tau_{t}$ as $\tau_{t}=2.4,12,24,30,48$. The result is shown in Fig. 3.6.

Equation (3.2) of the transfer function for the relative humidity system was used for tuning a transfer function to obtain the best matched model. The output of a simulation was graphed in a figure to match a real output by changing the parameter, $\tau_{h}$ and the parameter, $G_{h}$. After tuning the first-order model, the best values of $\tau_{h}$ and $G_{h}$ are $\tau_{h}=16$ and $G_{h}=1$, and the transfer function is given as

$$
T_{f h}(s)=\frac{1}{16 s+1} .
$$

The result of the best first-order RH model for a meat drying room is shown in Fig. 3.7. Two tests were done for the relative humidity system. The first test was tuning the parameter, $G_{h}$ in Equation (3.2) and assumed $\tau_{h}=16$. The test was done by changing $G_{h}$ as $G_{h}=0.5,0.75,1,1.25,1.5$. The result is shown in Fig. 3.8. The second test tuned the 


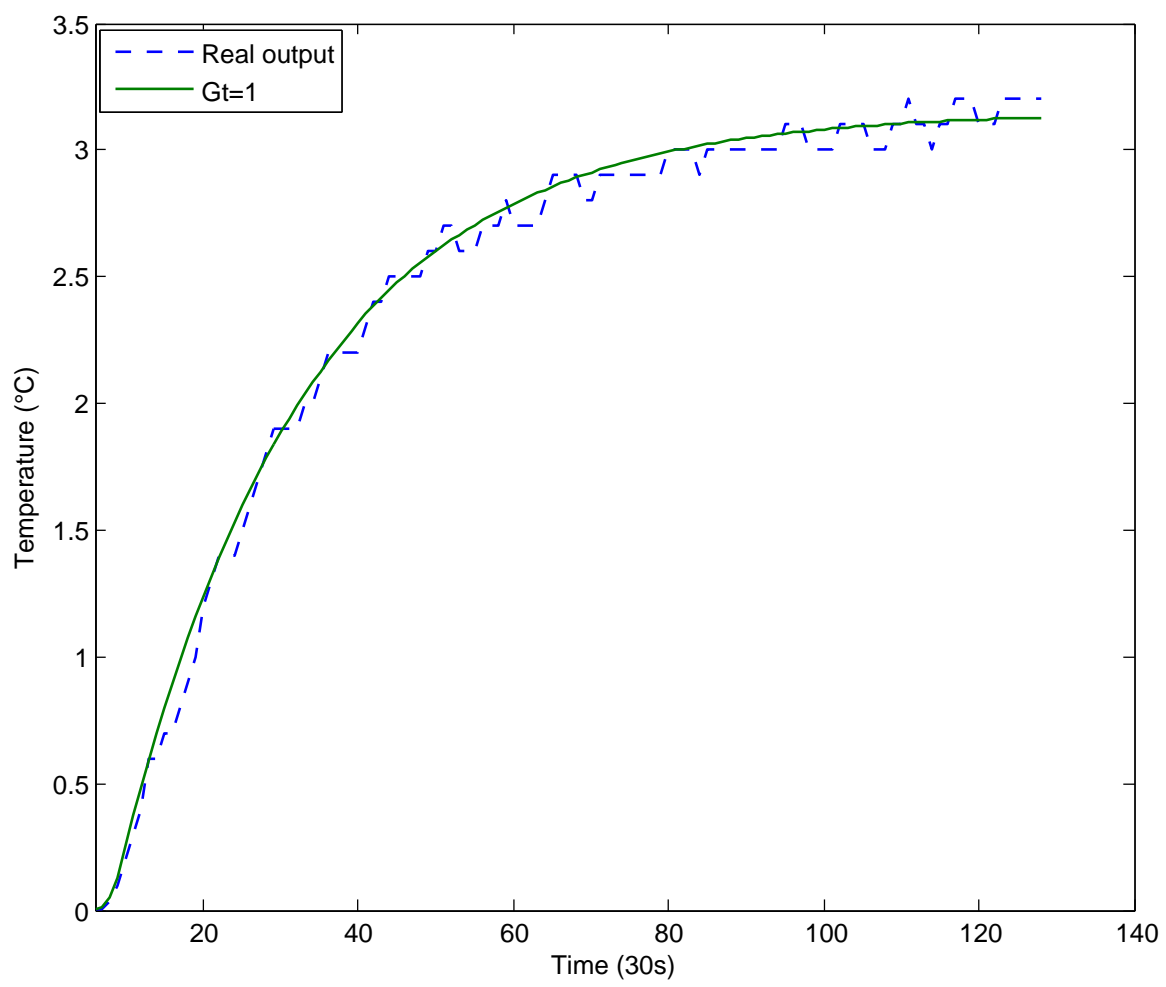

Figure 3.4: The best step responses of the first-order temperature model for a meat drying room.

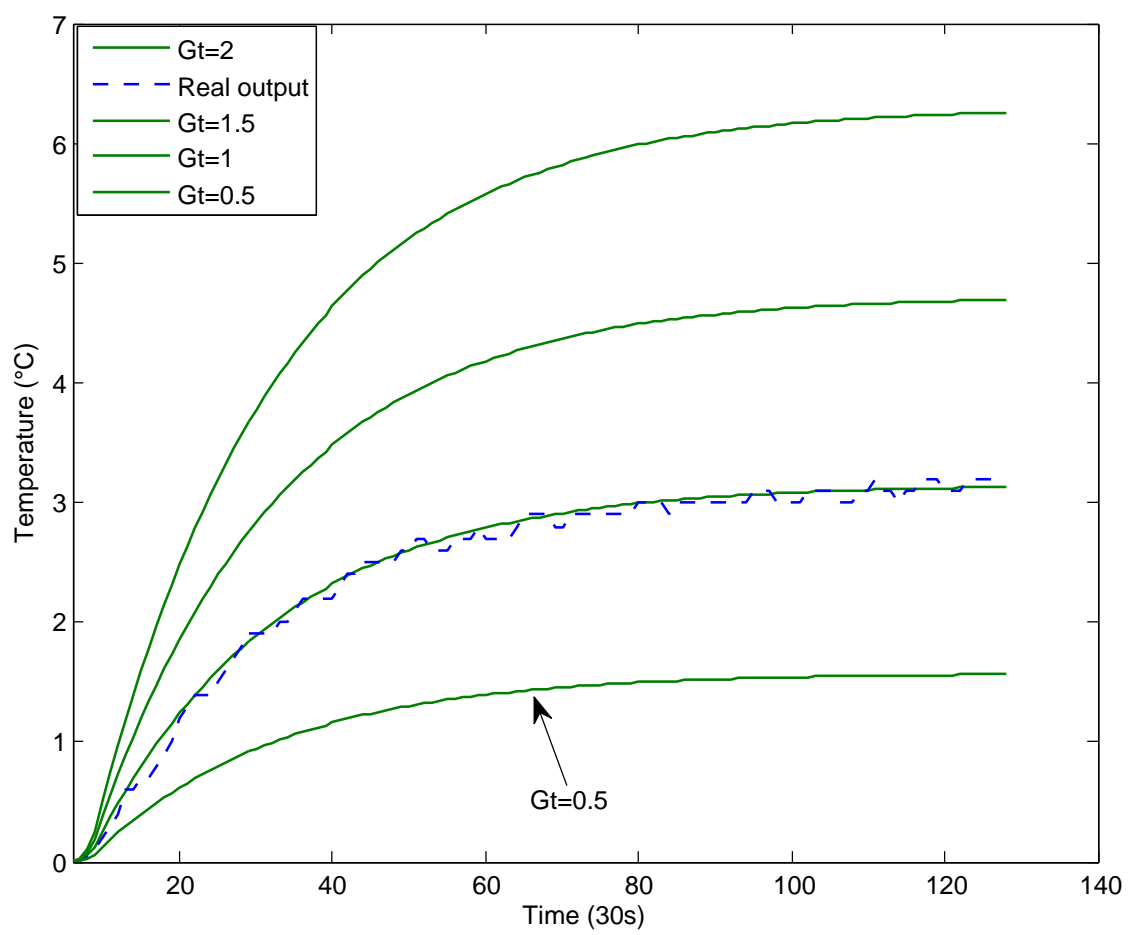

Figure 3.5: The step responses by changing $G_{t}$ in the first-order temperature model. 


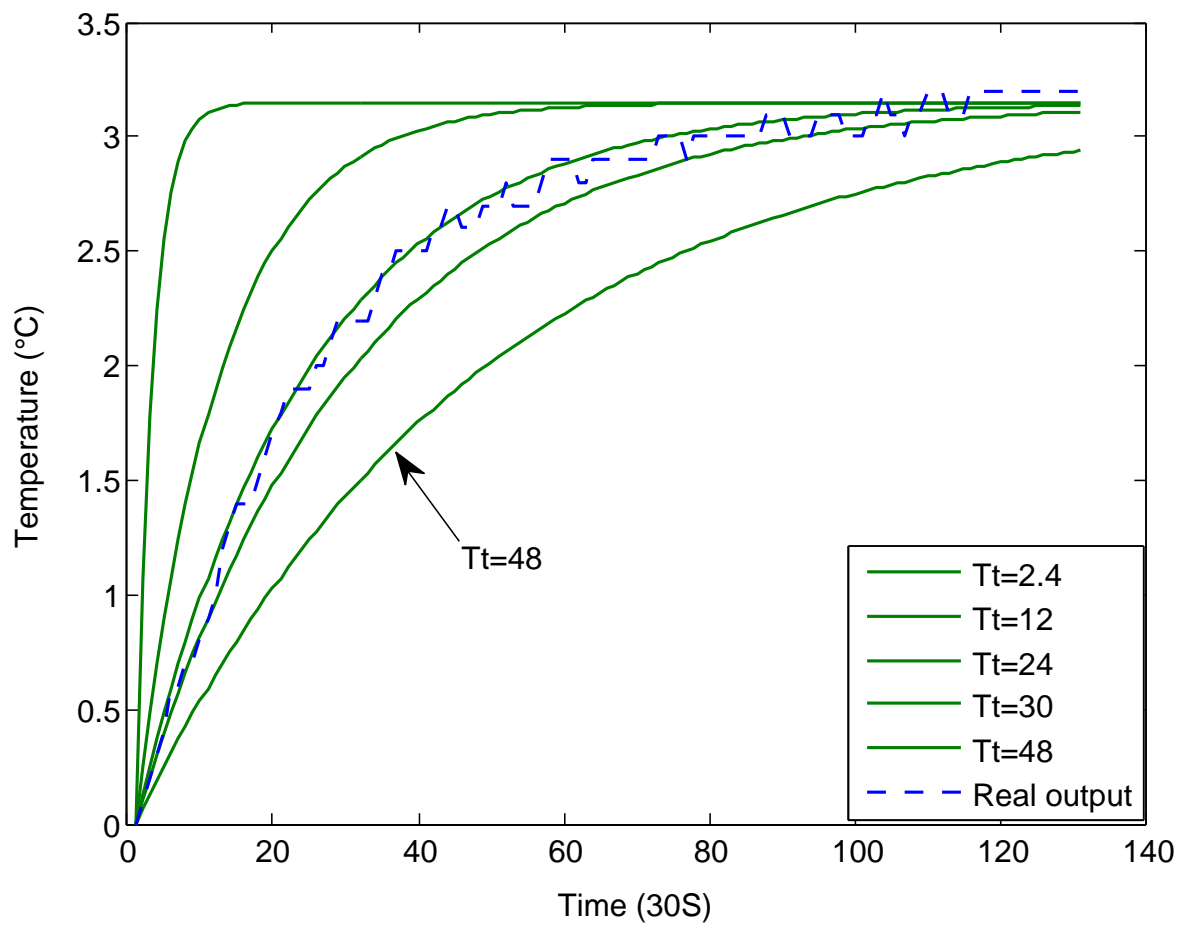

Figure 3.6: The step responses by changing $\tau_{t}$ in the first-order temperature model.

parameter, $\tau_{h}$ in Equation (3.2) and assumed the $G_{h}=1$ is the best value that was obtained from the first test. This test was done by changing $\tau_{h}$ as $\tau_{h}=8,12,16,20,24$. The result is shown in Fig. 3.9.

2. Second-order system experiments:

The $2 \xi \omega_{n}$ and $\omega_{n}$ in Equations (3.3) and (3.4) had been selected for the initial values, then the $2 \xi \omega_{n}$ and $\omega_{n}$ repeated changes for tuning the coefficient of transfer function equations, which was the same as tuning the transfer functions in first-order models in order to closely follow the values of real data from a meat drying room as shown in Figs. 3.2 and 3.3. Tuning the coefficients for a system condition with the poles placed on the left hand side of the $s$ plane, which is a controllable system for temperature and relative humidity. Therefore, the final transfer function equations for temperature and relative humidity are as follows.

1. Temperature system identification 


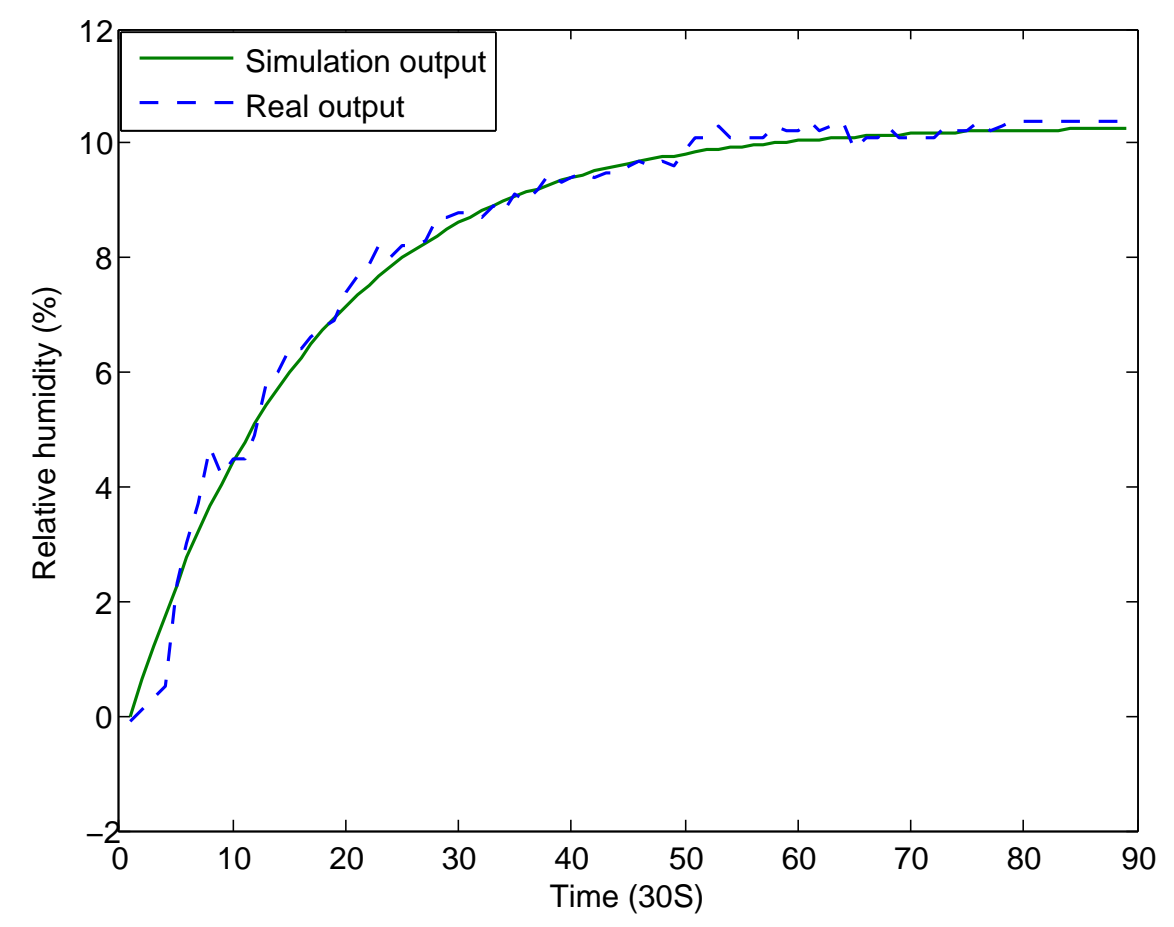

Figure 3.7: The best step responses of the first-order $\mathrm{RH}$ model for a meat drying room.

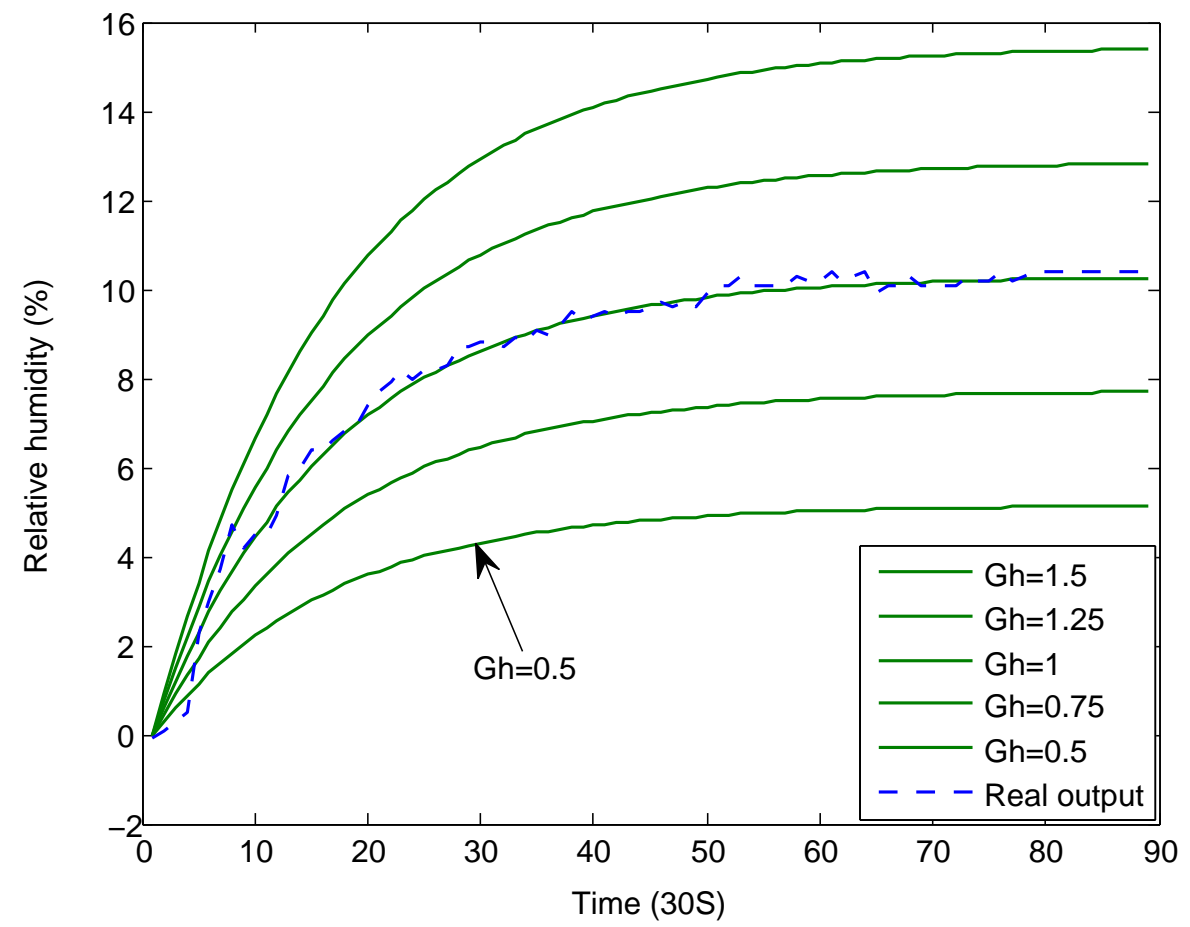

Figure 3.8: The step responses by changing $G_{h}$ in the first-order RH model. 


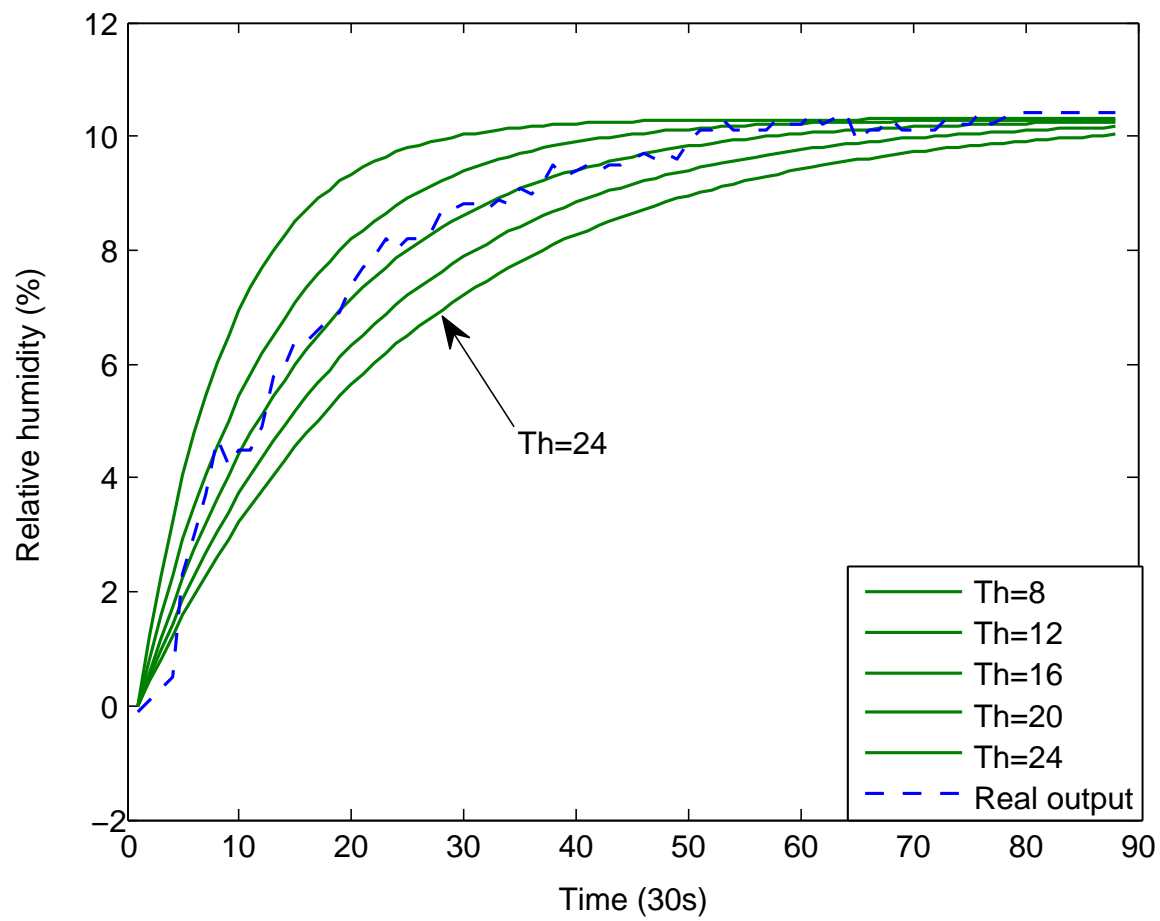

Figure 3.9: The step responses by changing $\tau_{h}$ in the first-order RH model.

Equation (3.3) of the transfer function of temperature with the best tuned is given as

$$
T_{t}(s)=\frac{1}{s^{2}+24 s+1}
$$

2. Relative humidity system identification

Equation (3.4) of the transfer function of relative humidity with the best tuned is given as

$$
T_{f h}(s)=\frac{1}{s^{2}+16 s+1} .
$$

The model of a meat drying room was programmed in Matlab for transfer functions. Models were constructed for temperature and relative humidity systems. Simulation results from these models were compared with an output of the real data from a meat drying room. Figs. 3.10 and 3.11 are simulation results of temperature and relative humidity compared with the real data in Figs. 3.2 and 3.3.

As shown in Fig. 3.10, the output of simulation models for temperature closely traces the output of the real data from a meat drying room. These matched the requirements of systems for temperature. As shown in Fig. 3.11, the output of simulation models of relative 


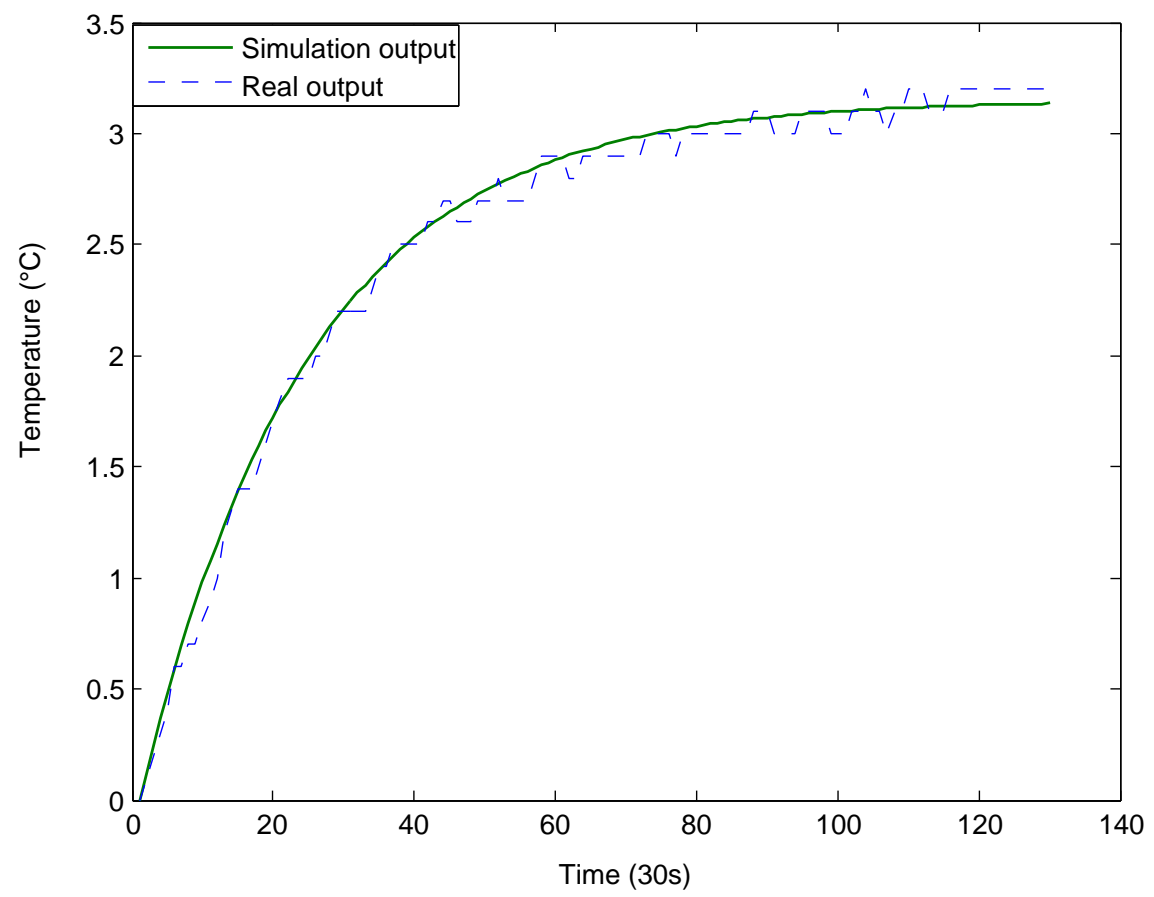

Figure 3.10: The best step responses of the second-order temperature model for a meat drying room.

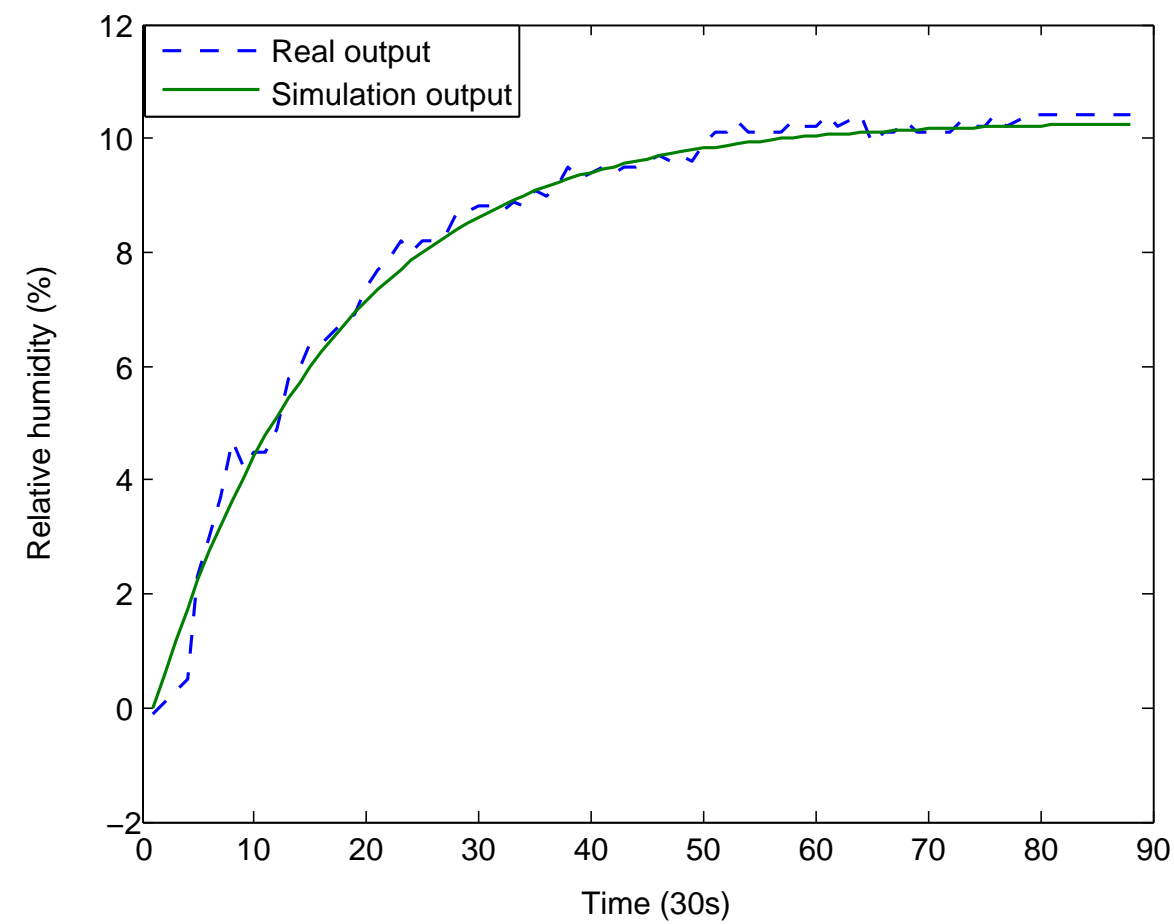

Figure 3.11: The best step responses of the second-order relative humidity model for a meat drying room. 
humidity closely traces the system output of the real data for a relative humidity in a meat drying room. These matched the requirements of systems for relative humidity.

The stability of the first order for a dynamic system in the continuous-time model is evaluated by the asymptotic behavior of a system when a time, $t$, tends toward infinity, and the initial input condition is started from zero (Landau and Zito, 2006). A condition of the stability is when $t \rightarrow \infty$, (1) for $\tau>0$ and $s<0$, the stable output asymptotically tend toward zero; (2) for $\tau<0$ and $s>0$, the output stability will diverge (Landau and Zito, 2006). A pole of the first- order transfer function of Equation (2.4) is calculated as $s=-\frac{1}{\tau}$.

A pole of the first-order transfer function of Equation (3.13) for the temperature system is $\tau_{t}=24$, and $s=-\frac{1}{\tau_{t}}=-\frac{1}{24}$. As the result, the temperature system of a meat drying system is stable. A pole of the first-order transfer function of Equation (3.14) for the relative humidity system is $\tau_{h}=16$, and $s=-\frac{1}{\tau_{h}}=-\frac{1}{16}$. As the result, the relative humidity system of a meat drying system is stable.

The stability of the second-order transfer function for a dynamic system in the continuoustime model is evaluated by the damping factor, $\xi$. A equation of the second-order transfer function is given as (Landau and Zito, 2006)

$$
Y_{s}(s)=\frac{\omega_{n}^{2}}{s^{2}+2 \xi \omega_{n} s+\omega_{n}^{2}} R_{s}(s),
$$

where $R_{s}(s)=\frac{1}{s}$. If $|\xi|<1$, roots of the transfer function poles are calculated as

$$
s_{1,2}=-\xi \omega_{n} \pm j \omega_{n} \sqrt{1-\xi^{2}}
$$

and the poles are complex poles; if $|\xi| \geq 1$, poles are calculated as

$$
s_{1,2}=-\xi \omega_{n} \pm \omega_{n} \sqrt{\xi^{2}-1}
$$

and the poles are real poles.

The value of the damping factor, $\xi$ determines the stability of the second-order system in the above two equations. If the $\xi>0$, the output will be an asymptotically stable system. If the $\xi<0$, the output will be an unstable system.

Poles of the second-order transfer function of Equation (3.15) for the temperature system were calculated by

$$
s^{2}+24 s+1=0
$$


Since $2 \xi \omega_{n}=24, \xi=\frac{24}{2 \times 1}=12$ and $\xi>0$, the output of the temperature system is asymptotically stable.

Solving the above Equation, the poles are $s_{1}=-47.9, s_{2}=-0.05$. The two poles lie on the left side of the $s$ plane, so the temperature system is controllable.

Poles of the second-order transfer function of Equation (3.16) for the relative humidity system were calculated by

$$
s^{2}+16 s+1=0
$$

Since $2 \xi \omega_{n}=16, \xi=\frac{16}{2 \times 1}=8$ and $\xi>0$, the output of the relative humidity system is asymptotically stable.

Solving the above equation the roots are $s_{1}=-31.9, s_{2}=-0.1$. The two poles lie on the left side of the $s$ plane, so the system is controllable.

\subsubsection{Discrete-Time Models}

Equation (3.7) of the transfer function in a discrete-time model was used for tuning a transfer function to obtain the best matched values of temperature. The output of a simulation system was graphed in a figure to match the output of real experimental data by changing a parameter, $a_{1 t}$ and a parameter, $b_{1 t}$ at same time. If $T_{s}=0.5 \mathrm{mins}$, then $a_{1 t}$ is calculated by Equation (3.8), and $b_{1 t}$ is calculated by Equation (3.9). The related parameters were calculated and listed in Table 3.1.

Table 3.1: The list of parameters for tuning a temperature transfer function.

\begin{tabular}{|c|c|c|c|}
\hline$\tau_{t}$ & $\frac{T_{s}}{\tau_{t}}$ & $a_{1 t}$ & $b_{1 t}$ \\
\hline 4 & 0.125 & -0.875 & 0.125 \\
\hline 8 & 0.0625 & -0.937 & 0.0625 \\
\hline 12 & 0.0416 & -0.958 & 0.0416 \\
\hline 16 & 0.0312 & -0.968 & 0.0312 \\
\hline 20 & 0.025 & -0.975 & 0.025 \\
\hline
\end{tabular}

The best result is shown in Fig. 3.12. After tuning the $a_{1 t}$ and $b_{1 t}$, the best values of 


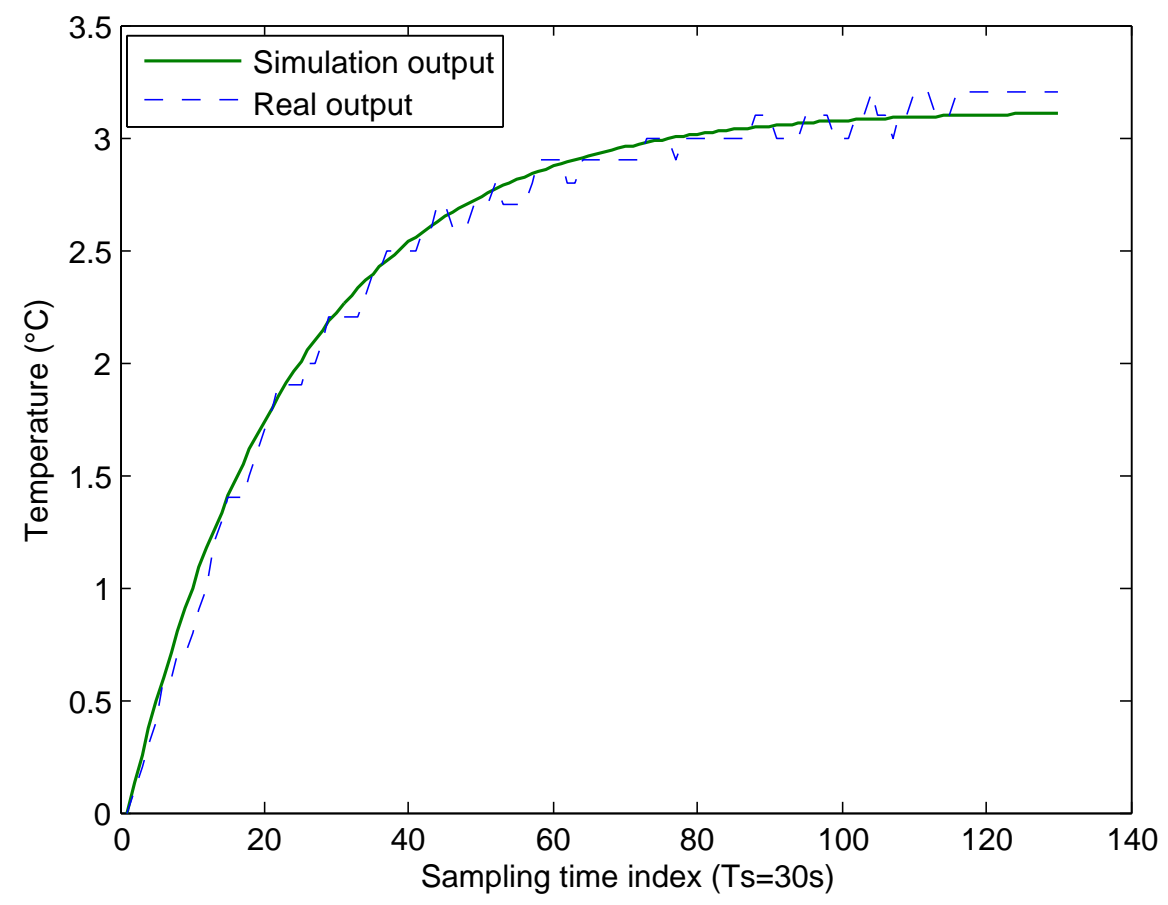

Figure 3.12: The best step responses of the discrete first-order temperature model for the drying room.

$a_{1 t}$ and $b_{1 t}$ for the equation of a transfer function is given as

$$
T_{t}(z)=\frac{0.0416}{z-0.958}
$$

where $T_{t}(z)$ is a transfer function of temperature. For obtaining the transfer function of temperature, the parameters, $a_{1}=-0.958$ and $b_{1}=0.0416$ were tuned for models of the first-order system to closely follow the real data in Fig. 3.13.

Equation (3.10) of a transfer function is for the relative humidity, which was used for tuning a transfer function to obtain the best matched values of relative humidity. The output of a simulation system was graphed in a figure to match the output of real data by changing a parameter, $a_{1 h}$ and a parameter, $b_{1 h}$ at same time. If $T_{s}=0.5 \mathrm{mins}$, then $a_{1 h}$ is calculated by Equation (3.11), and $b_{1 h}$ is calculated by Equation (3.12). The related parameters were calculated and listed in Table 3.2.

The result is shown in Fig. 3.14. After tuning the $a_{1 h}$ and $b_{1 h}$, the best values of $a_{1 h}$ and $b_{1 h}$ for the transfer function is given as

$$
T_{f h}(z)=\frac{0.0625}{z-0.937}
$$




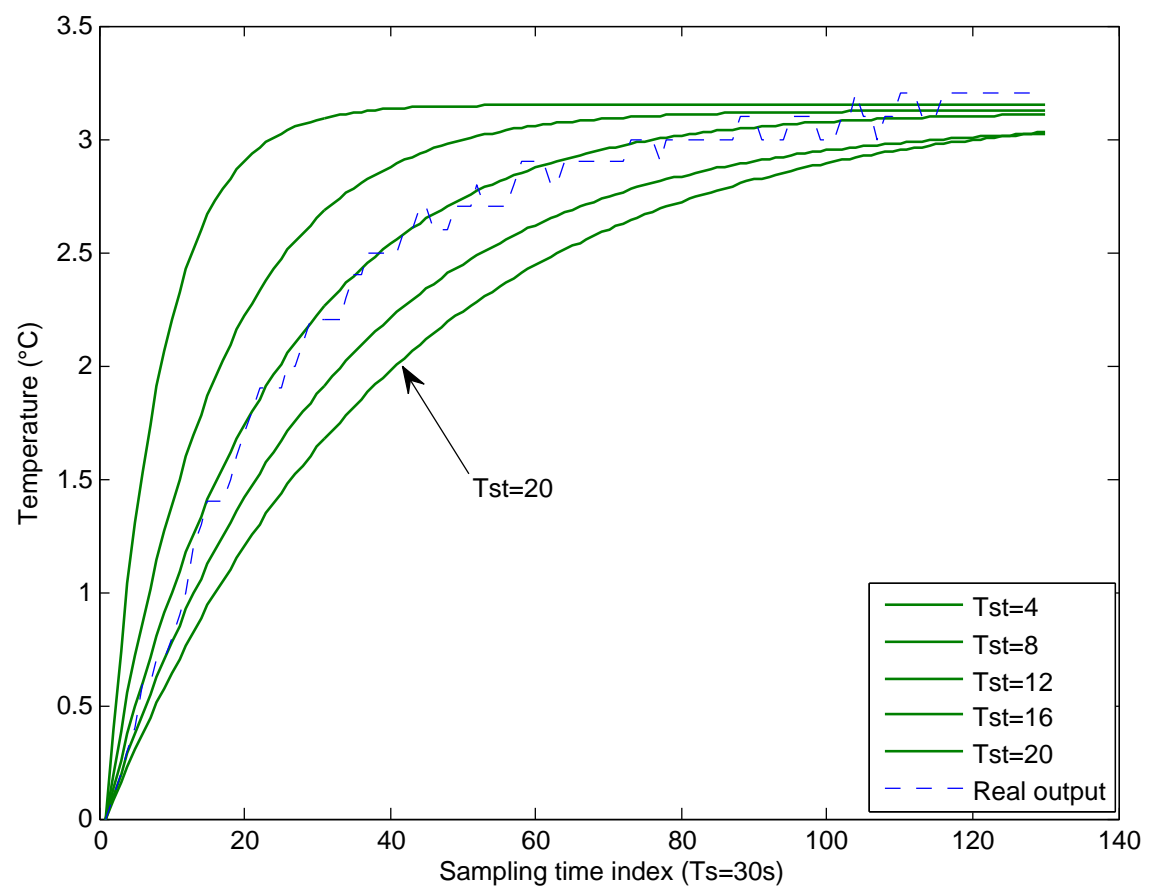

Figure 3.13: The step responses of by changing $T_{s}$ in the discrete temperature model for the drying room.

where $T_{f h}(z)$ is a transfer function of relative humidity. To obtain the transfer function of relative humidity, the parameters, $a_{1 h}=-0.937$ and $b_{1 h}=0.0625$ were tuned for models of the first-order system to match closely the real data as shown in Fig. 3.15.

The stability of discrete-time systems can be described by a parameter z. Equation (3.17) of temperature transfer function was obtained. Letting the denominator equal to zero gives

$$
1-0.958 z^{-1}=0, z=0.958<1
$$

therefore, the transfer function of temperature is asymptotically stable since the root of temperature transfer function denominator is inside of the unit circle in the $z$ plane.

Equation (3.18) of the relative humidity transfer function was obtained. Letting the denominator equals to zero gives

$$
1-0.937 z^{-1}=0, z=0.937<1
$$

therefore, the transfer function of relative humidity is asymptotically stable since the root of temperature transfer function denominator is inside of the unit circle in the $z$ plane. 


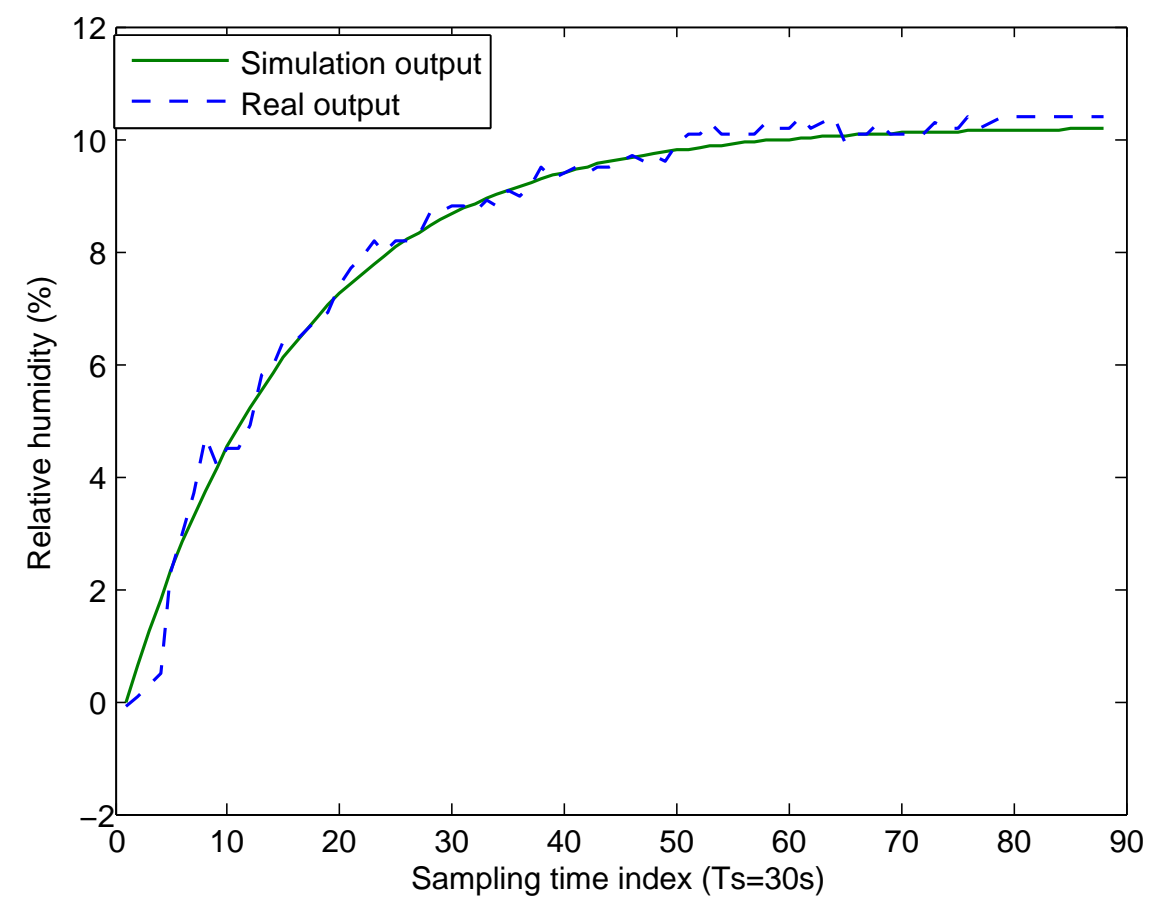

Figure 3.14: The best step response of the discrete first-order relative humidity model for a meat drying room.

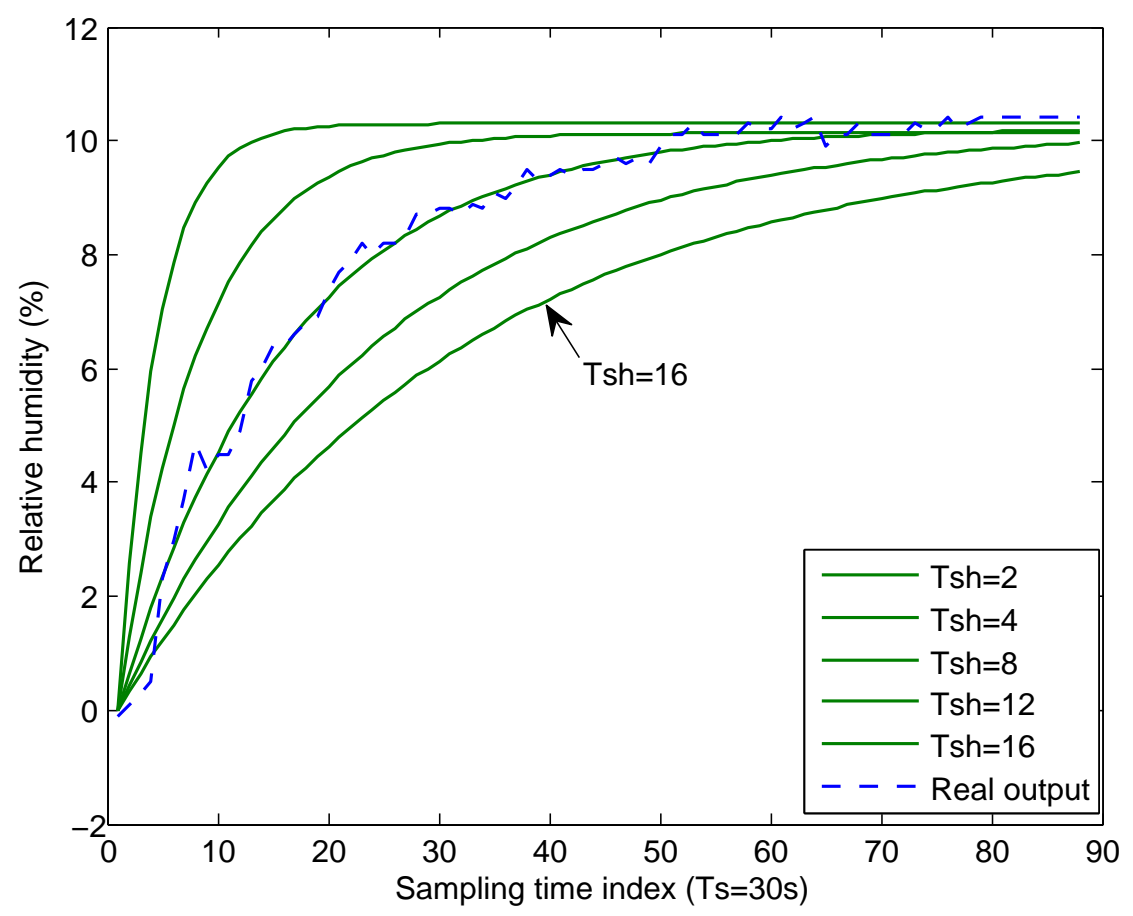

Figure 3.15: The step responses by changing $T_{s}$ in the discrete relative humidity model for a meat drying room. 
Table 3.2: The list of parameters for tuning a relative humidity transfer function.

\begin{tabular}{|c|c|c|c|}
\hline$\tau_{h}$ & $\frac{T_{s}}{\tau_{h}}$ & $a_{1 h}$ & $b_{1 h}$ \\
\hline 2 & 0.25 & -0.75 & 0.25 \\
\hline 4 & 0.125 & -0.875 & 0.125 \\
\hline 8 & 0.0625 & -0.937 & 0.0625 \\
\hline 12 & 0.0416 & -0.958 & 0.0416 \\
\hline 16 & 0.0312 & -0.968 & 0.0312 \\
\hline
\end{tabular}

\subsection{Comparison of the First-Order and the Second-Order Models}

The above experiments were performed in order to model the temperature and relative humidity systems. The transfer functions for continuous-time models were obtained by changing the time constant and the gain. The transfer functions for discrete-time models were obtained by changing the sampling time.

\subsubsection{Comparison of the Time Constant and the Damping Ratio}

To verify the time constants and damping ratios of transfer functions for temperature and relative humidity, the time constant and damping ratio for each transfer function were calculated. Time constants of the first-order system and damping ratios of the second-order system in continuous-time were calculated as follows.

For the temperature time constant in the first-order system, the max value of step responses for the temperature system was $V_{t m}=3.1$; the value of step responses of the temperature system at $63 \%$ was calculated as $V_{t 63}=1.953$. From Fig. 3.4, the time at $V_{t 63}=1.953$ was $T_{t t}=24.02$. Therefore, the time constant of the transfer function of temperature was calculated as $\tau_{t}=24.02$.

For the temperature damping ratio in the second-order system, the max value of step responses of the temperature was $V_{t m}=3.1$; the value of temperature at $63 \%$ was calculated as $V_{t 63}=1.953$. From Fig. 3.10, the time at $V_{t 63}=1.953$ was $T_{t t}=24.02$. Therefore, the damping ratio of the transfer function of temperature was calculated $\xi=12.01$. 
For the relative humidity time constant in the first-order system, the max value of step response of the relative humidity was $V_{h m}=10.2$; the value of relative humidity at $63 \%$ was calculated as $V_{h 63}=6.426$. From Fig. 3.7, the time at $V_{h 63}=6.426$ was as $T_{h t}=16.16$. Therefore, the time constant of the transfer function of relative humidity is calculated as $\tau_{h}=16.16$.

For the relative humidity damping ratio in the second-order system, the max value of step responses of the relative humidity was $V_{h m}=10.2$; the value of RH at $63 \%$ was calculated as $V_{h 63}=6.426$. From Fig. 3.11, the time at $V_{h 63}=6.426$ was $T_{h t}=16.16$. Therefore, the damping ratio of the transfer function of relative humidity was calculated $\xi=8.08$.

The experimental and calculated data for the time constants and the damping ratios are shown in Table 3.3.

Table 3.3: Experimental and calctulated time constants and the damping ratios for temperature and relative humidity transfer functions.

\begin{tabular}{|c|c|c|c|c|}
\hline & Experimental data & Calculation data & Experimental data & Calculation data \\
\hline Methods & First-order $(\tau)$ & First-order $(\tau)$ & Second-order $(\xi)$ & Second-order $(\xi)$ \\
\hline Temperature & 24.02 & 24 & 12.01 & 12 \\
\hline RH & 16.16 & 16 & 8.08 & 8 \\
\hline
\end{tabular}

\subsubsection{Comparison of the Correlation Coefficients and Least Mean Square Errors}

The correlation coefficients between the first and second-order temperature and relative humidity transfer functions and measured data, in continuous and discrete-time, were calculated. The correlation coefficient formula is given as (Arcidiacono, 2014)

$$
r=\frac{n \sum_{i=1}^{n} x_{i} y_{i}-\sum_{i=1}^{n} x_{i} \sum_{i=1}^{n} y_{i}}{\sqrt{\left(n \sum_{i=1}^{n} x_{i}-\left(n \sum_{i=1}^{n} x_{i}\right)^{2}\right)\left(n \sum_{i=1}^{n} y_{i}-\left(n \sum_{i=1}^{n} y_{i}\right)^{2}\right)}},
$$

where $n$ is the total number of samples, $x_{i}$ are the $x$ values, $y_{i}$ are the $y$ values.

The correlation coefficients are shown in Tables 3.4 for temperature and 3.5 for relative 
humidity. As shown, the correlation coefficients are very close to 1.0 . Therefore, Therefore, the simulated temperature and relative humidity data fits real measurements very well.

The Least Mean Square Error (LMSE) of the first-order or the second-order transfer functions for temperature and relative humidity in both continuous discrete-time were calculated. The MSE formula is given as (MSTE, 2015)

$$
E_{m s e}=\frac{1}{n} \sum_{i=1}^{n}\left(O_{i}-T_{i}\right)^{2}
$$

where $n$ is the total number of samples, $O_{i}$ are the objective values, $T_{i}$ are the target values.

The LMSE values are also shown in Tables 3.4 for temperature and 3.5 for relative humidity. The LMSE values also indicated a good match between the simulated and measured temperature and relative humidity data. In addition, note that the LMSE of the second-order model is lower than the LMSE of the first-order model in continuous-time.

Table 3.4: The comparison of the correlation coefficients and the LMSE values of temperature transfer functions.

\begin{tabular}{|c|c|c|c|}
\hline & Continuous-time model & Continuous-time model & Discrete-time model \\
\hline Methods & First-order & Second-order & First-order \\
\hline LMSE & 0.0047 & 0.0044 & 0.0054 \\
\hline $\mathrm{r}$ & 0.9972 & 0.9958 & 0.9967 \\
\hline
\end{tabular}

Table 3.5: The comparison of the correlation coefficients and the LMSE values of relative humidity transfer functions.

\begin{tabular}{|c|c|c|c|}
\hline & Continuous-time model & Continuous-time model & Discrete-time model \\
\hline Methods & First-order & Second-order & First-order \\
\hline LMSE & 0.0775 & 0.0761 & 0.0791 \\
\hline $\mathrm{r}$ & 0.9950 & 0.9951 & 0.9954 \\
\hline
\end{tabular}




\subsection{Summary}

In this chapter, A number of approaches for modelling temperature and relative humidity based on measured data was studied and considered both continuous-time and discretetime transfer functions. Simulated step responses based on the derived models closely matched the real measurement data of a meat drying system, for both temperature and relative humidity. Statistical analysis using both the correlation coefficient and LMSE also indicate very good match between the models and real systems. Therefore, the system models will be used to design control systems of temperature and relative humidity in later chapters. 


\section{Chapter 4}

\section{The Proposed Model for Coupling}

To propose a model of coupling, the root cause of this coupling must be identified, and the properties of coupling signals have to be analyzed. This chapter presents a model of coupling for meat drying systems according to real data of temperature and relative humidity obtained from real meat drying control systems. Usually, models are based on mathematical equations, however, there were no mathematical models to be found in the literature review section for coupling for meat drying room control systems. Therefore, the model presented here is based on fuzzy logic and neural networks, which simulates complex and nonlinear human thinking. This chapter will describe coupling analysis, propose methods for coupling, and report on simulation studies.

\subsection{Introduction}

The coupling of temperature and relative humidity is generated by temperature fluctuations and causes relative humidity fluctuations. Temperature fluctuations are directly affect the output of relative humidity in a meat drying control system. This research project studies the coupling after the controlled temperature under steady- state control only. Otherwise, coupling is ignored for the meat drying control system. Also coupling is a concern if the temperature fluctuations are outside of $\pm 0.1^{\circ} \mathrm{C}$. If the temperature fluctuations are inside of $\pm 0.1^{\circ} \mathrm{C}$, it is not necessary to decouple because the coupling signal is a unregulated signal. 
To identify the coupling, a model of ANFIS will be used. To build a complete control system for a meat drying room, a model of the coupling will be used.

\subsection{Coupling Identification}

Normally, a relative humidity control system is designed as an independent control loop. However, in practice, temperature fluctuations lead to relative humidity fluctuations in the control system. The temperature fluctuations act as coupling in relative humidity control system. These fluctuations are caused by coupling between the temperature and relative humidity. Coupling is related to relative humidity fluctuations. A number of factors such as the humidifier output, measurement signal drift, and disturbance in the controlled environment (e.g. foot traffic) can affect these fluctuations, and the relative humidity fluctuations are directly proportional to the fluctuation of temperature values.

The advantages of improving control accuracy of temperature and relative humidity for a meat drying control system are not only to increase the product quality but also reduce energy consumption. To identify coupling signals, experiments were conducted for a meat drying room control system.

\subsubsection{Experiment One}

Experiment one was done in a meat drying room. The set-point of room temperature was $13^{\circ} \mathrm{C}$, and the set-point of room relative humidity was $73 \% \mathrm{RH}$. The control algorithm used a PID algorithm for both control systems of temperature and relative humidity. Steam and refrigeration systems were used in the control systems to regulate the temperature and the relative humidity at specific set-points. A psychrometer was used as an instrument for temperature and relative humidity measurements in the meat drying room control system. Fig. 4.1 shows the coupling effects of temperature and relative humidity in a current meat drying room control system. 


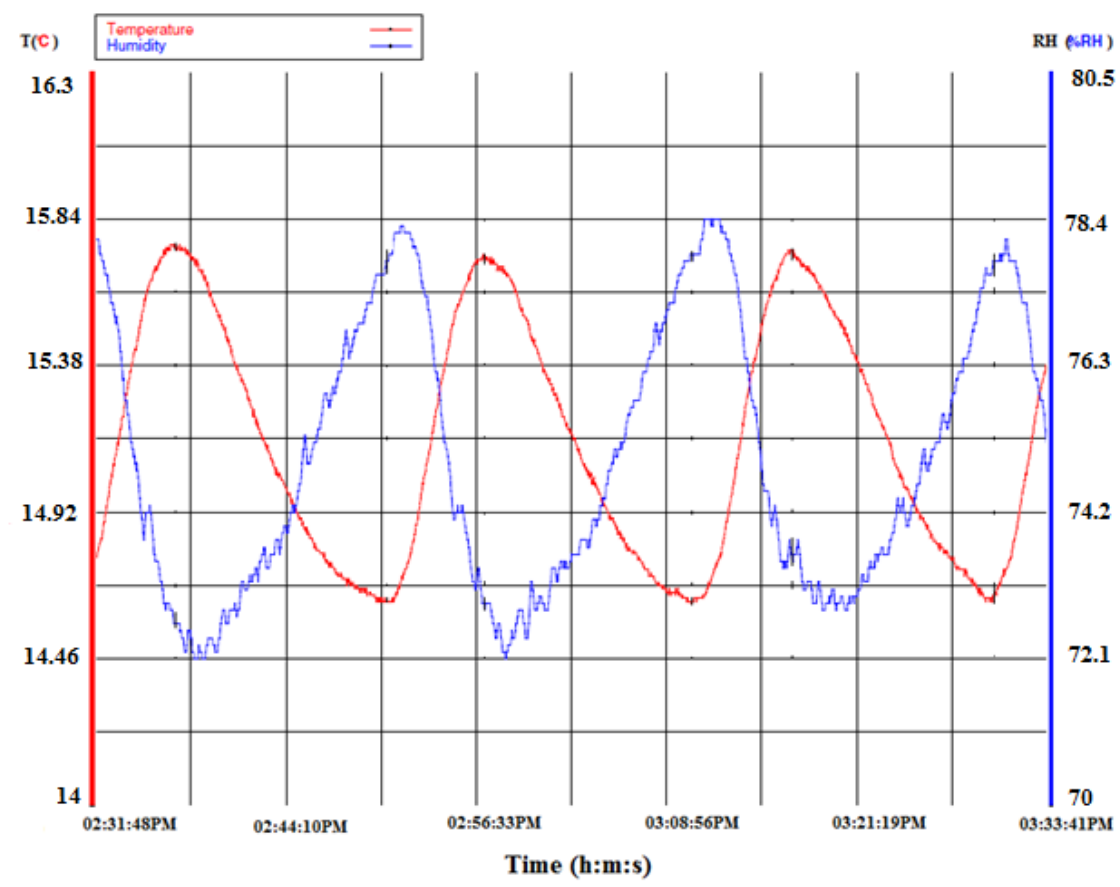

Figure 4.1: A coupling signal from the current meat drying room.

\subsubsection{Experiment Two}

Experiment two was done in a different way. To identify a relationship between coupling temperature and coupling relative humidity, a simulation was performed using ANFIS model, and the model was programmed with m code script in Matlab. The coupling temperature input of FIS model is designed with 9 input membership functions. The ranges of 9 input membership functions before running ANFIS model are shown in Fig. 4.2. The ranges of 9 input membership functions after running ANFIS model are shown in Fig. 4.3. The relationship between coupling temperature and coupling relative humidity is identified using ANFIS model, and a result is shown in Fig. 4.4. The dot curve in Fig. 4.4 is a simulation output of ANFIS model. The dot curve also shows that the relationship of coupling temperature and coupling relative humidity is nonlinear. Therefore, ANFIS model is suitable for a nonlinear relation. The code of ANFIS model is the $\mathrm{m}$ code in Matlab.

Figure 4.4 shows a nonlinear relationship between coupling temperature and coupling relative humidity. There is no mathematical model that can describe the relationship as shown in Fig. 4.4. 


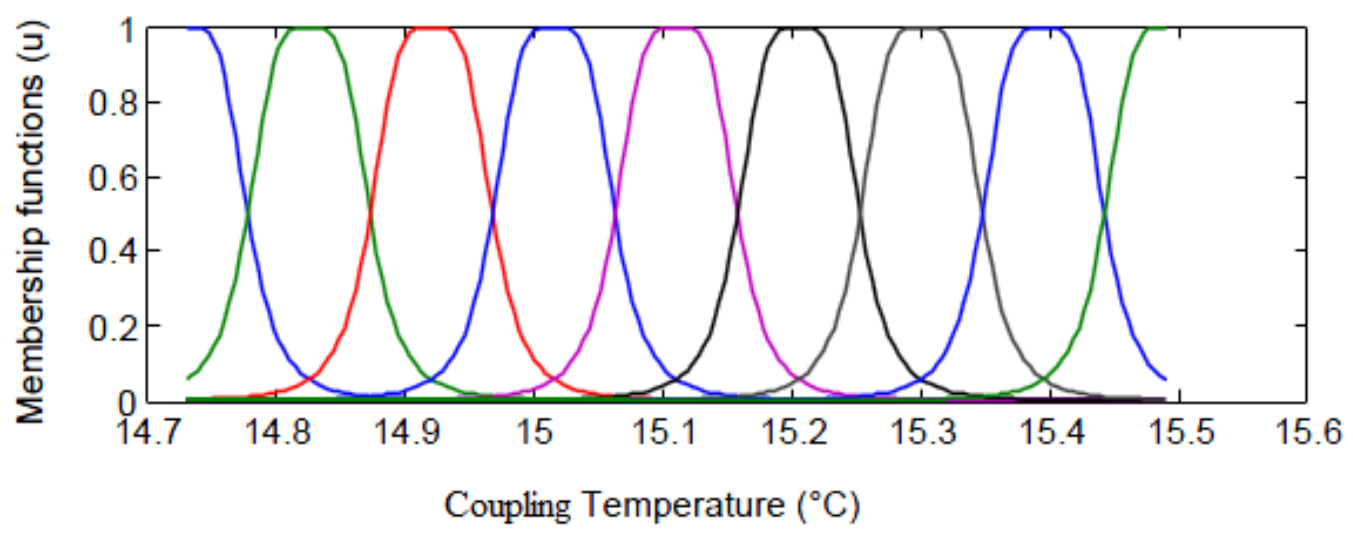

Figure 4.2: Input membership functions before the training of the ANFIS model.

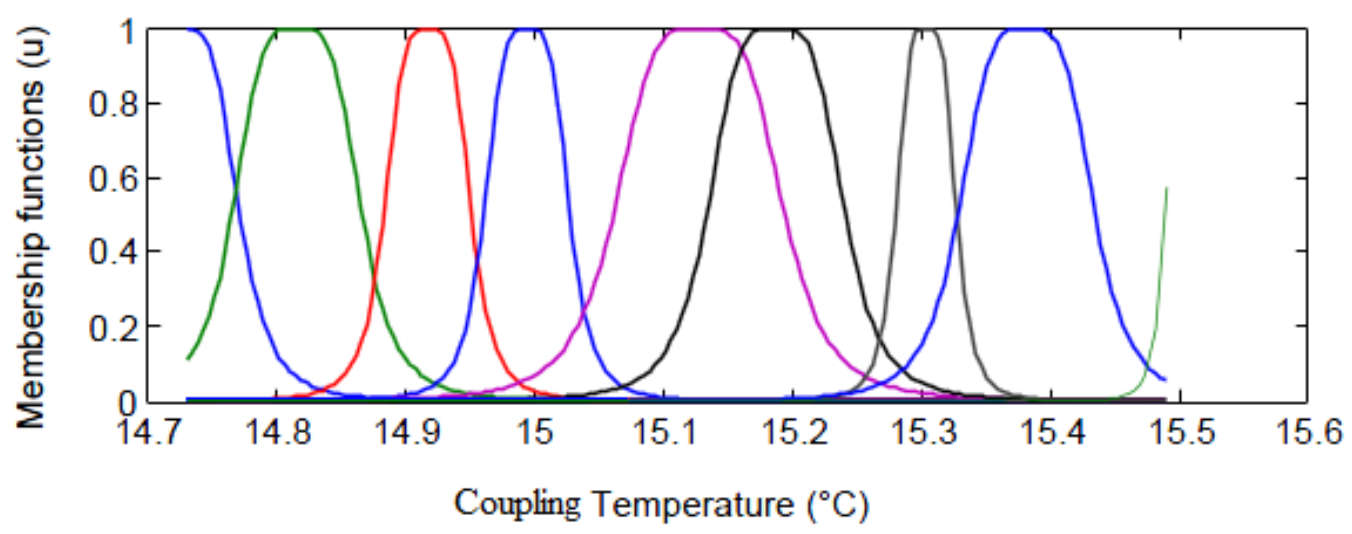

Figure 4.3: Input membership functions after the training of the ANFIS model. 


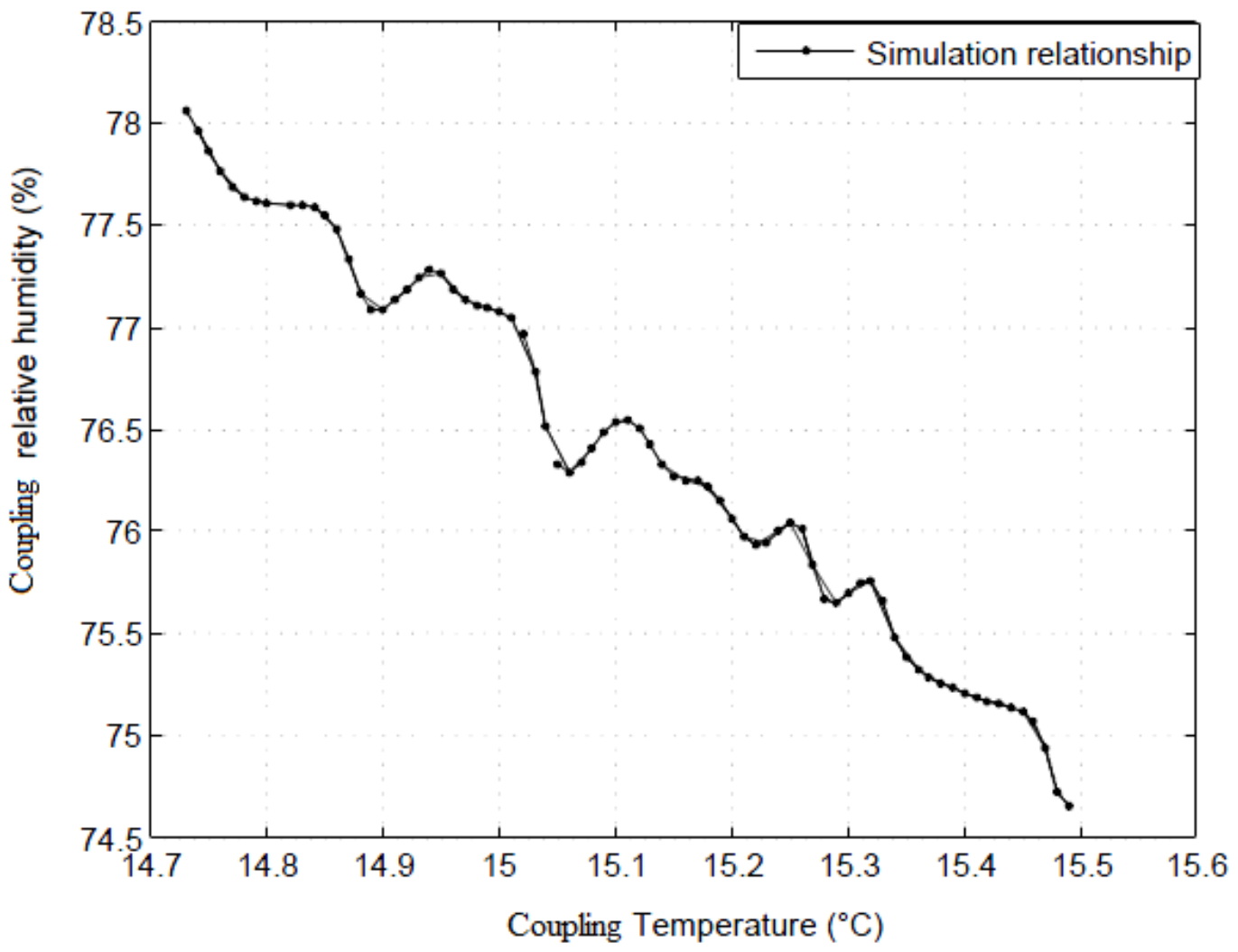

Figure 4.4: The relationship between coupling temperature and coupling relative humidity.

\subsubsection{Analysis}

As the above two experiment shows, a coupling of temperature and relative humidity certainly exists in the meat drying room. Although the relative humidity control was designed as an independent control system, the relative humidity control loop was still influenced by coupling temperature fluctuations. Therefore, the temperature fluctuations were disturbance signals in the relative humidity control system, and the disturbance of coupling shows characteristics of a nonlinear and complex signal in Fig. 4.1.

\subsection{The Proposed Models}

The above two experiments have identified coupling effects of temperature and relative humidity in the meat drying room and also found that the coupling signal was nonlinear and complex. Therefore, to build a nonlinear model for control system simulations, a method of a nonlinear algorithm have to be used in order to reduce the coupling effect. 


\subsubsection{Models of the Coupling}

The structure of an adaptive network is a multilayer network. The proposed model of coupling is an input as temperature and an output as relative humidity. A measurement of ANFIS is a relative humidity error. Formula of the relative humidity error measure $E_{h p}$ for the $p$ th $(1 \leq p \leq P)$ input of training data is given as

$$
E_{h p}=\sum_{m=1}^{\sharp(L)}\left(T_{h(m, p)}-O_{h(m, p)}^{L}\right)^{2},
$$

where $E_{h p}$ is the $p$-th error measurement of relative humidity, $P$ is training data pairs, $p$ is an element of training data pairs, $m$ is the index of layers, $L$ is the total number of layers, $\sharp(L)$ is an number of layers, $T_{h(m, p)}$ denote the $m$-th position of $p$-th target output vector

of relative humidity, and $O_{h(m, p)}^{L}$ is the $m$-th position of an actual output vector of relative humidity. The overall error measurement is given as

$$
E_{h}=\sum_{p=1}^{P} E_{h p}
$$

\subsubsection{Hybrid Learning Procedure}

Hybrid learning procedure for the proposed coupling model was used in ANFIS. Therefore, techniques of a fuzzy logic and neuron networks in ANFIS were also applied to a model of the nonlinear coupling signal. The results of the simulations will be shown in the next section.

The proposed model of coupling is based on learning paradigms for adaptive networks batch learning in ANFIS. The proposed hybrid learning procedure of the batch learning paradigm combines the gradient method and the least squares estimate to identify parameters. A forward pass and a backward pass consisted of each epoch used in the proposed hybrid learning procedure of ANFIS. The consequent parameters were identified by the least squares estimate for the proposed coupling model. The backward pass of the hybrid learning algorithm in the proposed coupling model was applied to the error rates backward propagation. The premise parameters could be updated by gradient descent. In the proposed model of the coupling, one input is as temperature, and one output is as relative humidity. There were three rules made for fuzzy inference systems. The network structure 


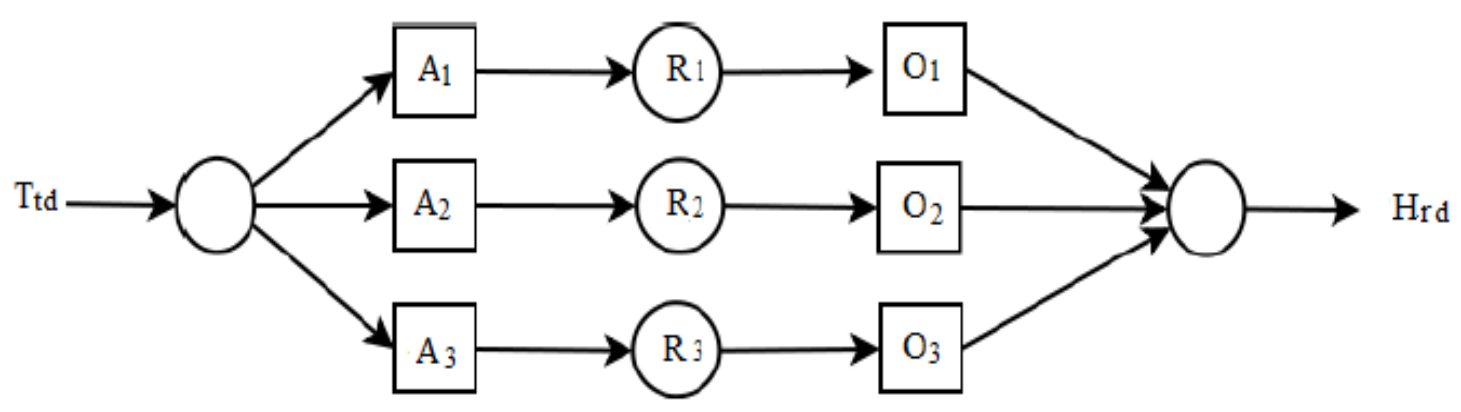

Figure 4.5: The network structure of ANFIS.

of ANFIS is shown in Fig. 4.5, where the input is disturbance temperature $T_{t d}$, the $A_{1}, A_{2}$, and $A_{3}$ are the sets of the data, the $R_{1}, R_{2}$, and $R_{3}$ are the fuzzy rules, the $\mathrm{O}_{1}, \mathrm{O}_{2}, \mathrm{O}_{3}$ are the output functions, and $\mathrm{H}_{r d}$ is the output of disturbance relative humidity.

Fuzzy three rules applied to a proposed model of the coupling signal were given as

1) IF $A_{1}$ is negative large (NL), THEN $\mathrm{O}_{1}$ is positive large (NL),

2) IF $A_{2}$ is zero $(Z)$, THEN $O_{2}$ is zero $(Z)$,

3) IF $\mathrm{A}_{3}$ is positive large (PL), THEN $\mathrm{O}_{3}$ is negative large (PL).

The fuzzy inference system of the proposed model for the coupling signal was linked a pair of the input and output data obtained from a real meat drying room system to train ANFIS tool in MATLAB. In order to fit proper fuzzy inference systems for the proposed model of the coupling signal, an experimental approach was conducted by examining the desired input and output data.

\subsubsection{ANFIS Architecture}

The proposed model of the coupling as architecture is 3 rules and 5 layers. At the following, each layer will be given in details:

1. Layer 1: the node of the layer 1 is a set of temperature data of the fuzzy membership function. The formula is given as

$$
O_{t i}^{1}=\mu_{A_{i}}\left(x_{t}\right), \quad i=1,2,3,
$$

where $x_{t}$ is a variable of a membership function, $A_{i}$ are fuzzy sets, and $\mu_{A_{i}}$ are the membership functions. 
If membership functions select a Gaussian function, the maximum value is equal to 1 , or the minimum value is equal to 0 . The formulas are given as

$$
\mu_{A_{i}}\left(x_{t}\right)=\exp \left\{-\left(\frac{x_{t}-c_{t i}}{a_{t i}}\right)^{2}\right\}, \quad i=1,2,3
$$

where $a_{t i}$ and $c_{t i}$ are the parameters in layer 1 which are as premise or input parameters.

2. Layer 2: The node of layer 2 is output the firing strenght of a rule. The formula is given as

$$
w_{i}=\mu_{A_{i}}\left(x_{t}\right), \quad i=1,2,3
$$

where $w_{i}$ are the firing strenght of a rule.

3. Layer 3: The node of layer 3 is a ratio. The formula is given as

$$
\overline{w_{i}}=\frac{w_{i}}{\sum w_{i}}, \quad i=1,2,3,
$$

where $\overline{w_{i}}$ is normlized firing strenghts.

4. Layer 4: The node of layer 4 is a square node with a node function of relative humidity. The formula is given as

$$
O_{h i}^{4}=\bar{w}_{i} f_{h i}, \quad i=1,2,3,
$$

where $f_{h 1}, f_{h 2}$, and $f_{h 3}$ are output functions of Takagi and Sugeno type of fuzzy systems; parameters in the layer 4 are called consequent parameters.

5. Layer 5: The node of layer 5 calculates the overall outputs of relative humidity. The formula is given as

$$
O_{h i}^{5}=\frac{\sum_{i} w_{1} f_{h i}}{\sum_{i} w_{i}}, \quad i=1,2,3
$$

The overall output functions of relative humidity can be calculated as a linear combination of the consequent parameters and the given values of temperature are premise parameters. The output of relative humidity is given as

$$
f_{h}=\sum \overline{w_{i}} f_{h i}, \quad i=1,2,3,
$$


where

$$
f_{h i}=\sum\left(x_{t i} p_{h i}+r_{h i}\right), \quad i=1,2,3,
$$

and $p_{h i}$ and $r_{h i}$ are the consequent parameters.

Rewitten the equation to show the overall output functions of relative humidity is given as

$$
f_{h}=\sum\left(\overline{w_{i}} x_{t i}\right) p_{h i}+\sum\left(\overline{w_{i}}\right) r_{h i}, \quad i=1,2,3 .
$$

The output function of relative humidity is given as

$$
H\left(x_{t}\right)=f_{h}\left(S_{t}\right),
$$

where the set of input variables of temperature is denoted by $S_{t}, H(\cdot)$ is an identity function of fuzzy inference system, and $f_{h}(\cdot)$ is a function of fuzzy inference system.

In the above descriptions, the methods for a fuzzy inference such as Sugeno fuzzy inference and Tsukamoto fuzzy inference were applied into the simulations of the proposed model for the coupling signal in a fuzzy tool in Matlab. The Gaussian membership function for the shape of member functions was used in the proposed model simulations of the coupling signal in the fuzzy tool in Matlab.

\subsection{Simulation Results}

The proposed model of the coupling signal was based on an adaptive neuro fuzzy inference system (ANFIS) tools in Matlab software for simulating a nonlinear coupling signal.

The procedures of the proposed model simulations for the coupling signal using ANFIS tool were as follows:

1. Prepare the pair of real data (temperature and relative humidity) for training neural networks in ANFIS tools.

2. Open the fuzzy tool in Matlab and selected new, then Sugeno.

3. Set up the membership functions of input disturbance temperature and selected Gaussian algorithm as active membership functions shown in Fig. 4.6. Set up parameters 
of disturance temperature as NL in a range of [14.7 15]; set up parameters of disturbance temperature as $\mathrm{Z}$ in a range of [14.9 15.3]; and set up parameters of disturbance temperature as PL in a range of [15.2 15.5]. Set up the over all of disturbance temperature in a range of [14.7 15.5].

4. Determine distrubance temperature fuzzy rules.

5. Set up the membership functions of output disturbance relative humidity and selected linear as active membership functions. Set up parameters of disturbance relative humidity as NL in a range of [73 75]; set up parameters of disturbance relative humidity as $\mathrm{Z}$ in a range of [74 78]; and set up parameters of disturbance relative humidity as $\mathrm{PL}$ in a range of [76 80]. Set up disturbance relative humidity in a range of [73 80].

6. Find the ANFIS in menu of the Edit and then open up an ANFIS window.

7. Load the data of disturbance temperature and disturbance relative humidity that were prepared in the first step for training testing. The training data were $80 \%$ out of 500 data, and the testing data were $20 \%$ out of 500 data.

\section{Generate FIS.}

9. Select a hybrid training method for training ANFIS with 100 epochs . The root mean square error changes is ploted in Fig. 4.7.

10. Train ANFIS. The training result is shown in Fig. 4.8.

11. Test ANFIS. The testing result is shown in Fig. 4.9.

The value of disturbance relative humidity is calculated by changing the input of disturbance temperature. The input disturbance temperature of membership functions after running ANFIS for a model of the coupling signal shows in Fig. 4.10. Comparing with Fig. 4.6, the membership functions of input disturbance temperature have an obvious change.

A algorithm of the root mean square error was used for the calculation training error and are shown in Fig. 4.7. The root mean square error after 100 epochs is 0.27385 . The model of the coupling showed fluctuations and was added into a simulation control system 


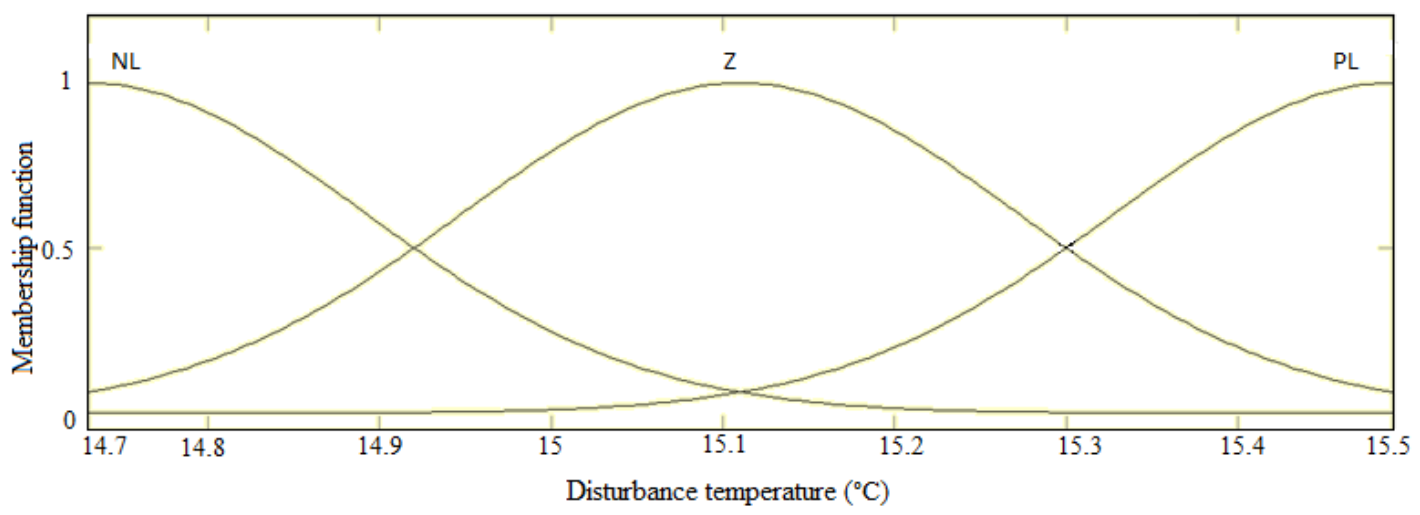

Figure 4.6: The membership functions of input disturbance temperate before the training of ANFIS for a model of the coupling signal.

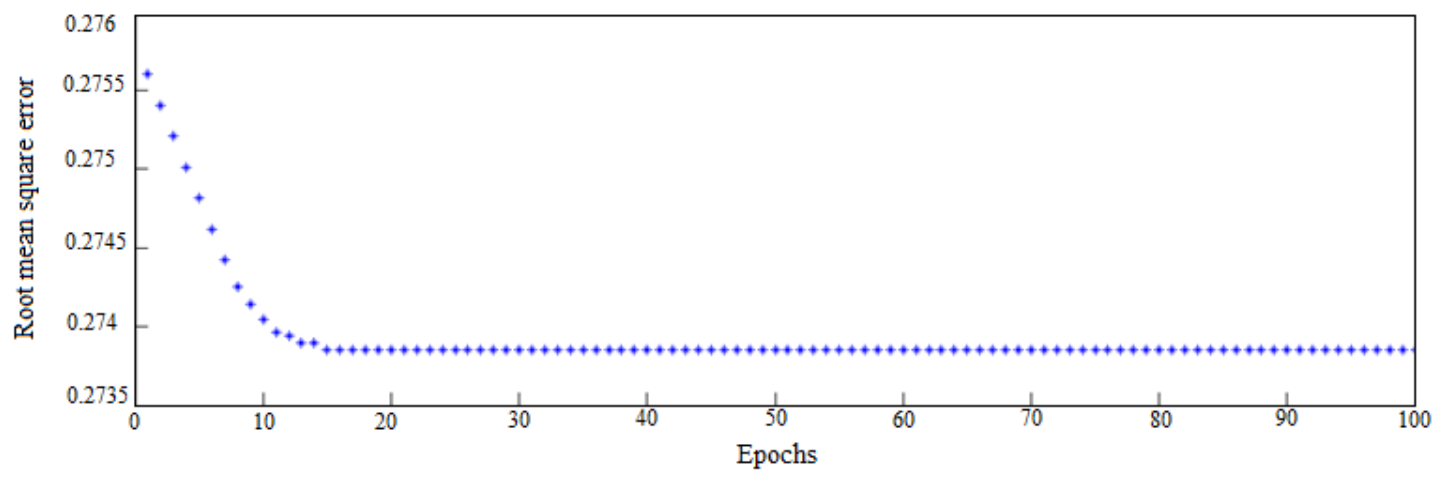

Figure 4.7: The ANFIS RMSE changing results.

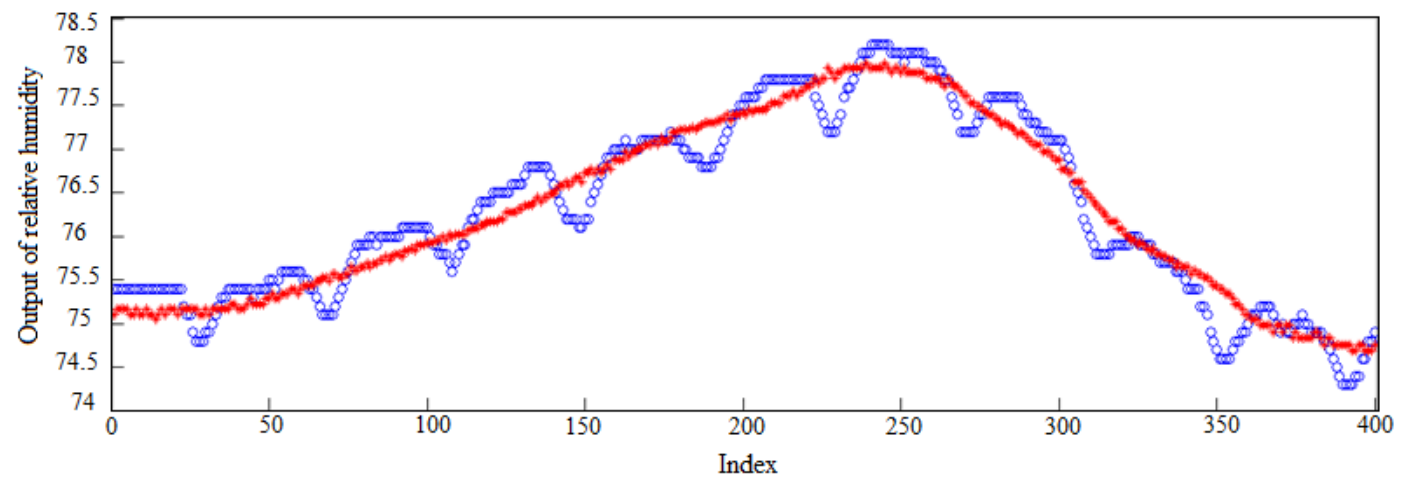

Figure 4.8: The ANFIS training results. The blue curve is the real output, and the red curve is the simulation output. 


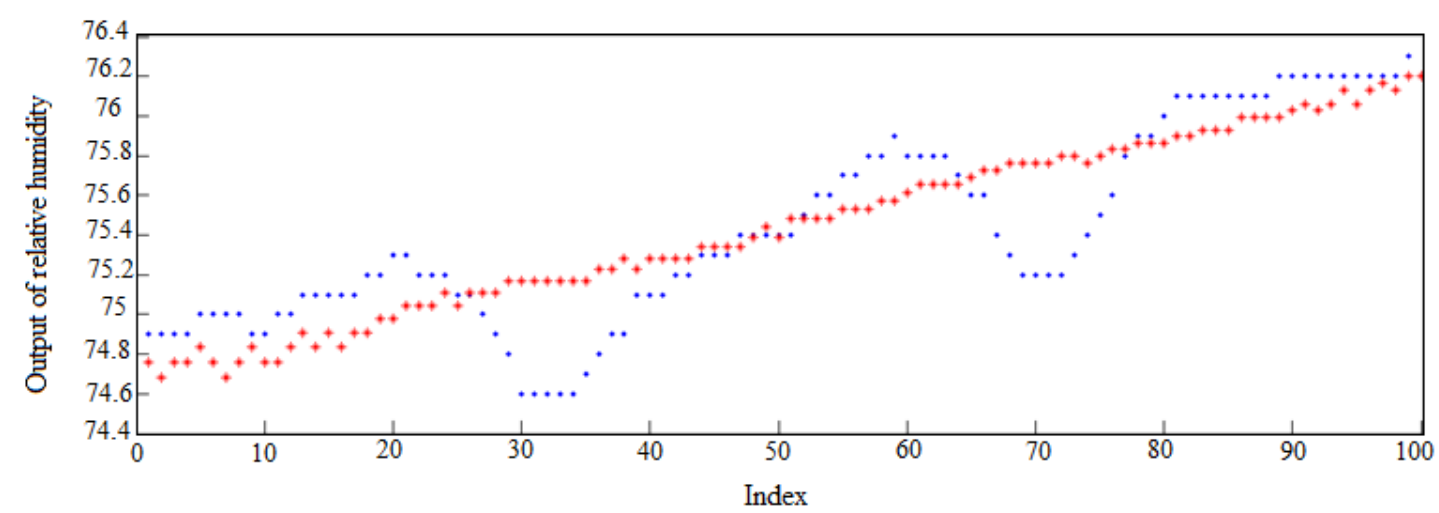

Figure 4.9: The ANFIS testing results. The blue curve is the real output, and the red curve is the simulation output.

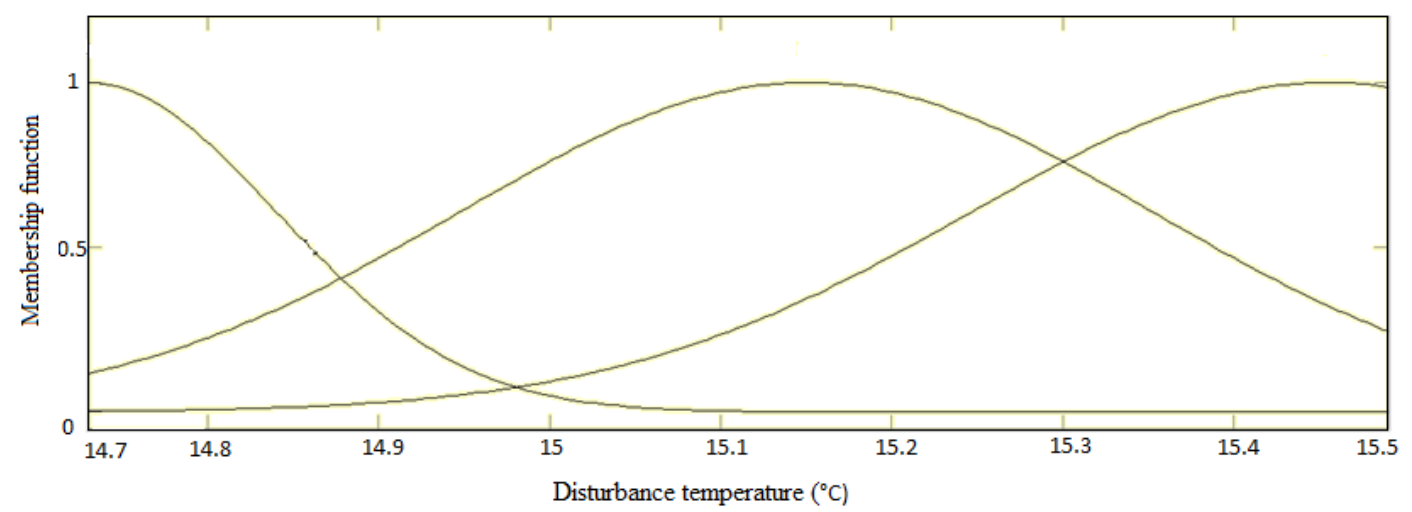

Figure 4.10: The membership functions of input disturbance temperate after the training of ANFIS for a model of the coupling signal.

for a meat drying room. The plot of the simulation model of coupling, and the plot of coupling signals of the real meat drying systems are shown in Fig. 4.11.

In Fig. 4.11, the plot with the dash line is the output of a simulation model of disturbace relative humidity, and the plot with solid line is the output of real meat drying systems of disturbance relative humidity. Both plots are closely followed that the coupling model is the most same as real disturbace relative humidity in a meat drying room. 


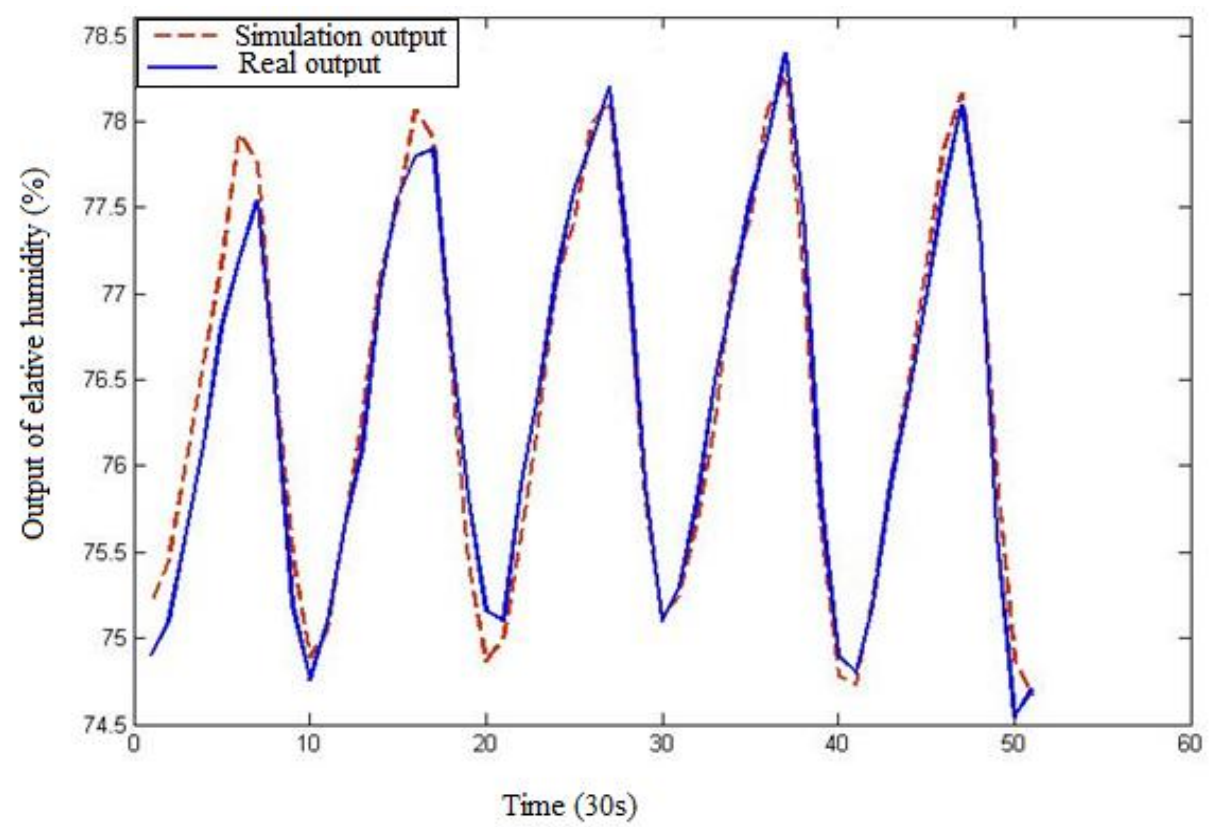

Figure 4.11: Comparison the simulation coupling signal with the real data from the meat drying room. The blue curve is the real output, and the red curve is the simulation output.

\subsection{Summary}

The coupling in temperature and relative humidity control systems has been identified and analyzed based on a real meat drying system. Two experiments were conducted to identify the coupling effects between temperature and relative humidity, and the results are shown in Figs. 4.1 and 4.4. For building the model of the coupling, the proposed method which using ANFIS model is suitable for nonlinear signals. The model of the coupling was simulated and tested using ANFIS tool in MatLab, and the results are shown in Fig. 4.8. In Fig. 4.8, the plot with dot-solid line is the output of the simulation model of relative humidity. 


\section{Chapter 5}

\section{The Proposed Control Methods for a Meat Drying Room}

The current control system of the meat drying room uses a PID control algorithm. A block diagram of the current system is shown in Fig. 3.1. The control performance of the system shows fluctuations of temperature and relative humidity exist in the meat drying room control system. Sometimes the fluctuations are great or less. Most of time, the system displays greater fluctuations. In practice, the coupling effects of the meat drying room control system displays nonlinearity (Fig. 4.1). Therefore, a linear PID control method cannot overcome fluctuations in a nonlinear system. Advanced control methods have to be involved to a control system.

\subsection{Introduction}

In experiments conducted earlier, a meat drying control system for a meat drying process was mainly to control the temperature and relative humidity of drying air. For controlling the temperature, relative humidity, and velocity of the air, systems of heating, ventilating and refrigeration system had been included in the meat drying room. A psychrometer was used as a measurement instrument for simultaneous control of temperature and relative humidity. In this section, a major topic of this research project focuses on methods for designing a control system for a meat drying room. A method of simultaneous control 
of temperature and relative humidity will be selected because the temperature and relative humidity are critical control parameters for the meat drying processing. The simultaneous control system is multivariable control (two input variables and two output variables), which can be named as Multi-Input and Multi-Output (MIMO) system.

Digital control systems are similar to analog control systems, which can be designed with a closed-loop control system. A transfer function of closed-loop control system using a digital controller canonical structure can be used. In digital control systems, digital PI is commonly used a controller. A digital PI controller can be designed by Equation (2.24) of PI controller.

This section will describe how a complete control system was designed to control the temperature and relative humidity using Simulink in MatLab software and simulate models. Also simulation results will be compared with the real data based on a current meat drying control system.

\subsection{The Proposed Control Methods}

A popular control method used in industry is the PID algorithm with a closed-loop feedback control system. In the proposed method, the PID controller or PI controller was designed with a continuous-time model and a discrete-time model. The fuzzy logic controller was also designed.

\subsubsection{Analog Controller Design}

A PID algorithm equation in a continuous-time model for a temperature controller is given as

$$
M_{t}(t)=K_{p t}+\frac{1}{K_{i t}} \int E_{t}(t) d t+K_{d t} \frac{d E_{t}(t)}{d t},
$$

to convert the time domain to frequency domain, rewritten Equation (5.1) is given as

$$
M_{t}(s)=K_{p t}+\frac{1}{K_{i t} s} E_{t}(s)+K_{d t} s E_{t}(s)
$$

where $K_{p t}$ is the proportional gain of the PID controller of temperature, $K_{i t}$ is the integral gain of the PID controller of temperature, $K_{d t}$ is the derivative gain of the PID controller 
of temperature, $M_{t}$ is the controlled output of, and $E_{t}$ is the error (a set point signal of temperature minus a feedback signal of temperature).

A PID control equation in continuous-time for a relative humidity controller is given as

$$
M_{h}(t)=K_{p h}+\frac{1}{K_{i h}} \int E_{h}(t) d t+K_{d h} \frac{d E_{h}(t)}{d t} .
$$

To convert the time domain to frequency domain, Equation (5.3) is rewritten as

$$
M_{h}(s)=K_{p h}+\frac{1}{K_{i h} s} E_{h}(s)+K_{d h} s E_{h}(s)
$$

where $K_{p h}$ is the proportional gain of the PID controller of relative humidity, $K_{i h}$ is the integral gain of the PID controller of relative humidity, $K_{d h}$ is the derivative gain of the PID controller of relative humidity, $M_{h}$ is the PID controller output, and $E_{h}$ is the error (a difference between a set point signal of relative humidity and a feedback signal of relative humidity). The manual tuning method had been proposed for tuning the PID controllers for temperature control system and relative humidity control system.

\subsubsection{Digital Controller Design}

The proposed digital PI controller of temperature was described in Equation (2.24), and the model of the temperature digital PI controller is given as

$$
u_{t}(t)=u_{t}(t-1)-r_{0 t} y_{t}(t)-r_{1 t} y_{t}(t-1)+r_{0 t} r_{t}(t)+r_{1 t} r_{t}(t-1)
$$

Equation (2.12) was used for designing a transfer function, and the model of the plant is given as

$$
T_{t}\left(z^{-1}\right)=\frac{b_{1 t}\left(z^{-1}\right)}{1+a_{1 t}\left(z^{-1}\right)},
$$

which can be rewritten as

$$
T_{t}(z)=\frac{b_{1 t}}{z+a_{1 t}},
$$

where

$$
\begin{gathered}
a_{1 t}=\frac{T_{s t}}{\tau_{t}}-1, \\
b_{1 t}=\frac{G_{t} T_{s t}}{\tau_{t}},
\end{gathered}
$$


Equations (2.25) and (2.26) to obtain parameters $r_{0 t}$ and $r_{1 t}$ for the digital PI controller are given as

$$
\begin{gathered}
r_{0 t}=G_{t}\left(1+\frac{\tau_{s t}}{\tau_{t}}\right) ; \\
r_{1 t}=-G_{t} .
\end{gathered}
$$

The closed-loop control system of the output $y_{t}(t)$ is given as

$$
y_{t}(t)=\frac{b_{1 t}}{z+a_{1 t}} u_{t}(t)
$$

The tuning of the temperature digital PI controller was to change the parameters, $r_{o t}$ and $r_{1 t}$. The parameters $a_{1 t}$ and $b_{1 t}$ were obtained by Equations (5.8) and (5.9) for the transfer functions of temperature.

The proposed digital PI controller of relative humidity used Equation (2.24), and the model of relative humidity digital PI controller is given as

$$
u_{h}(t)=u_{h}(t-1)-r_{0 h} y_{h}(t)-r_{1 h} y_{h}(t-1)+r_{0 h} r_{h}(t)+r_{1 h} r_{h}(t-1) .
$$

Equation (2.12) was used for designing the transfer function of relative humidity, and the model of the plant is given as

$$
T_{f h}\left(z^{-1}\right)=\frac{b_{1 h}\left(z^{-1}\right)}{1+a_{1 h}\left(z^{-1}\right)} .
$$

which can be rewritten as

$$
T_{f h}\left(z^{-1}\right)=\frac{b_{1 h}}{z+a_{1 h}}
$$

where

$$
\begin{aligned}
a_{1 h} & =\frac{T_{s h}}{\tau_{h}}-1, \\
b_{1 h} & =\frac{G_{h} T_{s h}}{\tau_{h}} .
\end{aligned}
$$

Equations (2.25) and (2.26) to obtain parameters $r_{0 h}$ and $r_{1 h}$ for the digital PI controller are given as

$$
\begin{gathered}
r_{0 h}=G_{h}\left(1+\frac{T_{s h}}{\tau_{h}}\right), \\
r_{1 h}=-G_{h} .
\end{gathered}
$$


The closed-loop control system of the output $y_{h}(t)$ is given as

$$
y_{h}(t)=\frac{b_{1 h}}{z+a_{1 h}} u_{h}(t)
$$

The tuning of the digital relative humidity digital PI controller was to change the parameters, $r_{o h}$ and $r_{1 h}$. The parameters $a_{1 h}$ and $b_{1 h}$ were obtained by Equations (5.16) and (5.17) for the transfer functions of relative humidity.

\subsubsection{Fuzzy Controller Design}

The proposed method for analog control systems of a meat drying room is designed to use a fuzzy control method in a continuous-time model, because it is better suited for control performances of the system.

Because systems of a meat drying room are two input variables and two output variables (temperature and relative humidity). The systems are proposed that have two feedback control loops independently, a temperature control loop and a relative humidity control loop. The relative humidity control loop is proposed to have a fuzzy control method, in order to reduce the fluctuations between temperature and relative humidity. As the coupling is nonlinear, using a fuzzy control method takes an advantage of a feature of fuzzy logic control to solve a nonlinear problem for the meat drying room system.

A proposed fuzzy logic controller of relative humidity is a single-input and a singleoutput structure. The input is an error of relative humidity, which is a difference between a set point and a feedback control signal. The output of the fuzzy logic controller of relative humidity is connected to an input of the PID controller.

Fuzzification is the first step of the proposed fuzzy logic controller, which is the input of observed data. Fuzzification is usually called crisp, and it maps the observed input space to fuzzy sets in a certain input.

The next step after the proposed fuzzification is inference systems. As shown in Fig. 5.1 , the inference systems are between the inputs and defuzzification functions in a fuzzy logic controller to perform an inference function. In a Mamdani-type fuzzy model, crisp values are used as inputs, and its defuzzification is to convert a fuzzy set to a crisp value.

The Mamdani-type fuzzy model was used for the proposed fuzzy logic controller. In the 


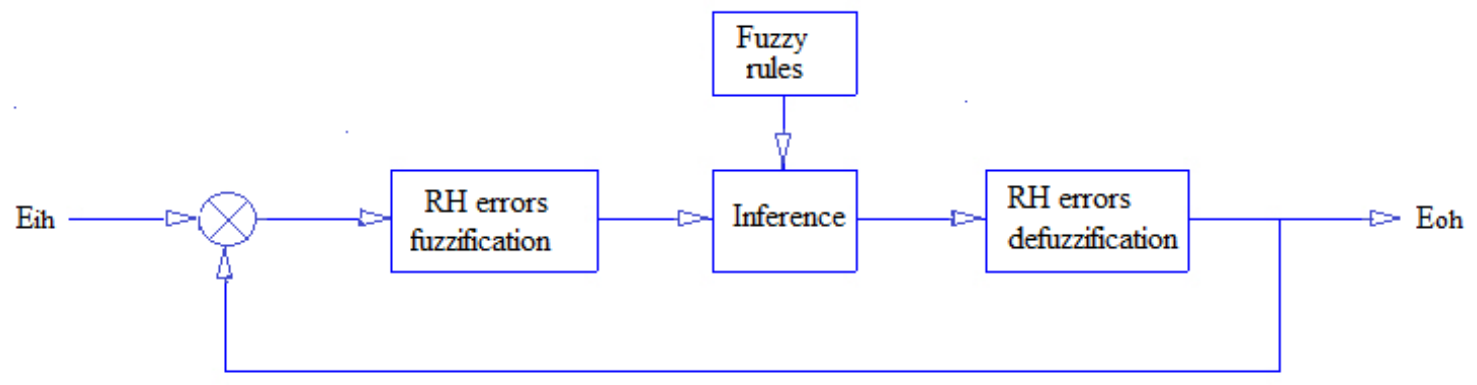

Figure 5.1: The proposed fuzzy logic controller of relative humidity.

proposed fuzzy logic controller of relative humidity, errors were crisp values as input, and defuzzification was used to convert a fuzzy set to a crisp value of errors.

Since inference systems are based on fuzzy control rules, and because "rule based" is important for a fuzzy logic controller, "rule based" is characterized by a set of linguistic statements and based on expert knowledge. The form of rules is "IF-THEN". In the simulation experiments of the proposed fuzzy logic controller of relative humidity, the errors $\left(\mathrm{E}_{i h}\right)$ of inputs were divided into a range of positive large (PS), zero (Z), negative small (NS), and negative large (NL). The errors of outputs $\left(\mathrm{E}_{o h}\right)$ were divided into a range of positive small (PS), zero (Z), negative small (NS), and negative large (NL). The rules of the error of relative humidity input are shown in Table 5.1.

Table 5.1: Fuzzy control rules of fuzzy PID control. NL: negative large; Z: zero; PL: positive large; NS: negative small; PS: positive small.

\begin{tabular}{|c|c|c|c|c|}
\hline & Rule 1 & Rule 2 & Rule 3 & Rule 4 \\
\hline IF E $_{i}=$ & NL & NS & Z & PS \\
\hline THEN E $_{o}=$ & PS & Z & NS & NL \\
\hline
\end{tabular}

\subsubsection{PI-like Fuzzy Logic Controller Design}

The proposed method for digital control systems used a PI-like fuzzy logic control model for control the temperature and relative humidity of a meat drying room. One PI-like fuzzy 
logic controller is for temperature, and one PI-like fuzzy logic controller is for relative humidity. A difference between proposed digital control systems and proposed analog control systems is a temperature controller.

\section{PI-like fuzzy logic controller for temperature}

The proposed method of PI-like fuzzy logic controllers for temperature and relative humidity used a Mamdani-type fuzzy inference. The fuzzy logic controller is designed to have two inputs of fuzzification and one output of defuzzification. One input is an error, $e$, and another input is a difference error, $\Delta e$. The "IF-THEN" rule form is used for the PI-like fuzzy logic controllers.

In the simulation experiments of the proposed PI-like fuzzy logic controller of temperature, the errors $\left(\mathrm{e}_{i t}\right)$ of temperature inputs were divided into a range of a positive large (PL), zero $(\mathrm{Z})$, and negative large $(\mathrm{NL})$. The errors of temperature outputs $\left(\mathrm{e}_{o t}\right)$ were divided into a range of a positive small (PS), zero (Z), and negative small (NS). The difference errors $\left(\Delta \mathrm{e}_{i t}\right)$ of inputs were divided into a range of a positive large (PL), zero (Z), and negative large (NL). The errors of output $\left(\mathrm{e}_{o t}\right)$ were divided into a range of a positive small (PS), zero (Z), and negative small (NS). The rules of the error and the difference error of temperature input are shown in Table 5.2.

Table 5.2: Fuzzy control rules of the temperature controller. NL: negative large; Z: zero; NS: negetive small; PS: positive small.

\begin{tabular}{|c|c|c|c|}
\hline Rules & $\mathrm{e}_{i t}$ & $\Delta \mathrm{e}_{i t}$ & $\mathrm{e}_{o t}$ \\
\hline $\mathrm{R}_{1}$ & $\mathrm{NL}$ & $\mathrm{NL}$ & $\mathrm{NS}$ \\
\hline $\mathrm{R}_{2}$ & $\mathrm{NL}$ & $\mathrm{Z}$ & $\mathrm{Z}$ \\
\hline $\mathrm{R}_{3}$ & $\mathrm{NL}$ & $\mathrm{PL}$ & $\mathrm{Z}$ \\
\hline $\mathrm{R}_{4}$ & $\mathrm{Z}$ & $\mathrm{NL}$ & $\mathrm{Z}$ \\
\hline $\mathrm{R}_{5}$ & $\mathrm{Z}$ & $\mathrm{Z}$ & $\mathrm{Z}$ \\
\hline $\mathrm{R}_{6}$ & $\mathrm{Z}$ & $\mathrm{PL}$ & $\mathrm{Z}$ \\
\hline $\mathrm{R}_{7}$ & $\mathrm{PL}$ & $\mathrm{NL}$ & $\mathrm{Z}$ \\
\hline $\mathrm{R}_{8}$ & $\mathrm{PL}$ & $\mathrm{Z}$ & $\mathrm{Z}$ \\
\hline $\mathrm{R}_{9}$ & $\mathrm{PL}$ & $\mathrm{PL}$ & $\mathrm{PS}$ \\
\hline
\end{tabular}




\section{PI-like fuzzy logic controller for relative humidity}

In the proposed PI-like fuzzy logic controller of relative humidity, the errors $\left(\mathrm{e}_{i h}\right)$ of inputs were divided into a range of positive large (PL), zero (Z), and negative large (NL). The errors of outputs $\left(\mathrm{e}_{o h}\right)$ were divided into a range of positive small (PS), zero $(\mathrm{Z})$, and negative small (NS). The difference errors $\left(\Delta \mathrm{e}_{i h}\right)$ of inputs were divided into a range of positive large (PL), zero $(\mathrm{Z})$, and negative large $(\mathrm{NL})$. The errors of output $\left(\mathrm{e}_{o h}\right)$ were divided into a range of positive small (PS), zero (Z), and negative small (NS). The rules of the error and the difference error of relative humidity input are shown in Table 5.4.

Table 5.3: Fuzzy control rules of the relative humidity controller. NL: negative large; Z: zero; NS: negetive small; PS: positive small.

\begin{tabular}{|c|c|c|c|}
\hline Rules & $\mathrm{e}_{i h}$ & $\Delta \mathrm{e}_{i h}$ & $\mathrm{e}_{o h}$ \\
\hline $\mathrm{R}_{1}$ & $\mathrm{NL}$ & $\mathrm{NL}$ & $\mathrm{NS}$ \\
\hline $\mathrm{R}_{2}$ & $\mathrm{NL}$ & $\mathrm{Z}$ & $\mathrm{Z}$ \\
\hline $\mathrm{R}_{3}$ & $\mathrm{NL}$ & $\mathrm{PL}$ & $\mathrm{Z}$ \\
\hline $\mathrm{R}_{4}$ & $\mathrm{Z}$ & $\mathrm{NL}$ & $\mathrm{Z}$ \\
\hline $\mathrm{R}_{5}$ & $\mathrm{Z}$ & $\mathrm{Z}$ & $\mathrm{Z}$ \\
\hline $\mathrm{R}_{6}$ & $\mathrm{Z}$ & $\mathrm{PL}$ & $\mathrm{Z}$ \\
\hline $\mathrm{R}_{7}$ & $\mathrm{PL}$ & $\mathrm{NL}$ & $\mathrm{Z}$ \\
\hline $\mathrm{R}_{8}$ & $\mathrm{PL}$ & $\mathrm{Z}$ & $\mathrm{Z}$ \\
\hline $\mathrm{R}_{9}$ & $\mathrm{PL}$ & $\mathrm{PL}$ & $\mathrm{PS}$ \\
\hline
\end{tabular}

\subsection{Simulation Results}

The fuzzy PID controller of relative humidity had been designed for the proposed method of a meat drying control system. Simulation experiments are essential steps for identifying control performance of systems. Three control methods had been designed, simulated, and described as below. 


\subsubsection{Analog Control Systems}

Analog control system used a continuous-time model for PID controllers. Control systems include two parts, without disturbance and with disturbance, for comparing the affects of a PID control method.

\section{PID control without disturbances}

The PID control method was proposed with a feedback control loop for the meat drying room control systems, in order to evaluate the systems modeling. The mathematical model of equations was used for obtaining the models of controllers. Equation (5.2) is used as the PID controller of temperature, and Equation (5.4) is used as the PID controller of relative humidity.

To tune a PID controller for controlling each individual temperature or relative humidity, a manual tuning method was used. First, let $K_{i t}=0$ and $K_{d t}=0$, then $K_{p t}$ was adjusted from large numbers to small numbers and tested, and the final value of the adjusted number was a proper $K_{p t}$ value. This value of $K_{p t}$ was kept and let $K_{d t}=0 . K_{i t}$ was added for tuning; and $K_{i t}$ was adjusted from large numbers to small numbers, and the final value of the adjusted number was a proper $K_{i t}$ value. Finally, $K_{d t}$ was tuned, and $K_{d t}$ was adjusted from large numbers to small numbers, and the final value of the adjusted number was a proper $K_{d t}$ value. The following equations for temperature and relative humidity are given as

$$
M_{t}(s)=0.99+\frac{1}{20 s} E_{t}(s)+2 s E_{t}(s)
$$

and

$$
M_{h}(s)=1+\frac{1}{22 s} E_{h}(s)+5 s E_{h}(s) .
$$

As shown in Fig. 5.2, the input is a step unit, and the set point of temperature is $15^{\circ} \mathrm{C}$. The output of temperature is $15^{\circ} \mathrm{C}$ without disturbances. Therefore, the PID controller of temperature works very well without disturbances. As shown in Fig. 5.3, the input is a step unit, and the set point of relative humidity is $76 \%$. The output of relative humidity is 76 $\%$ without disturbances. Therefore, the PID controller of relative humidity works without disturbances. 


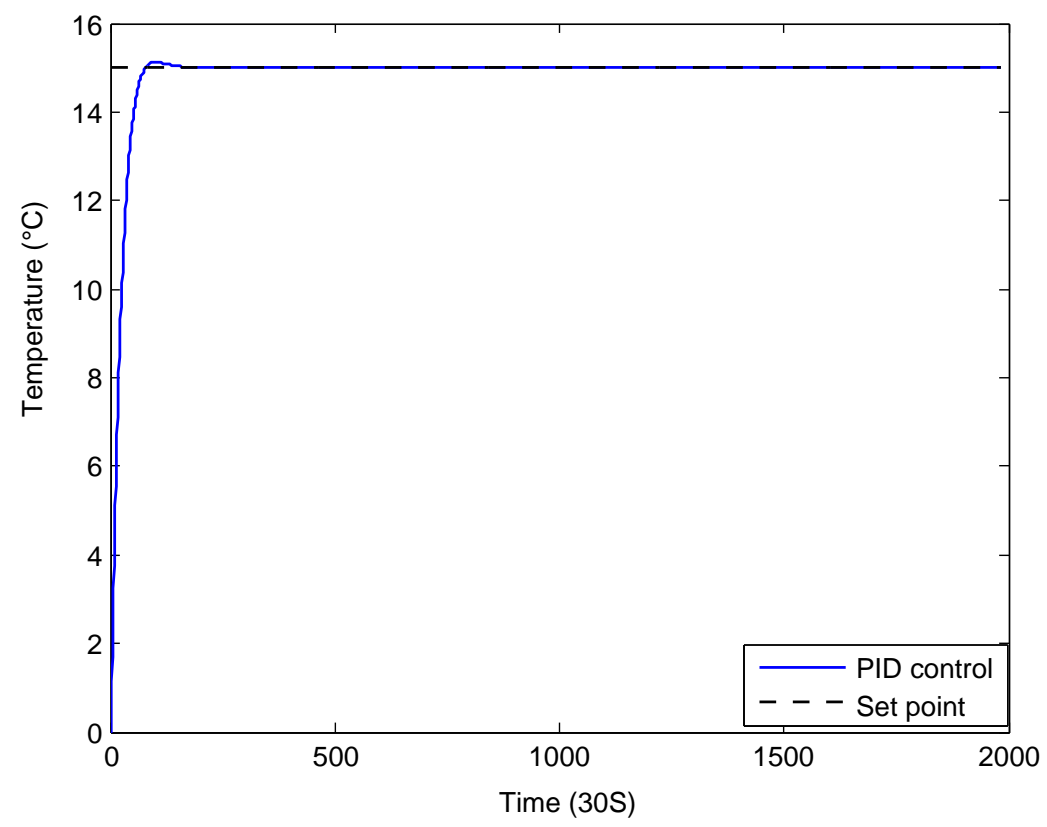

Figure 5.2: The output of PID control without temperature disturbances.

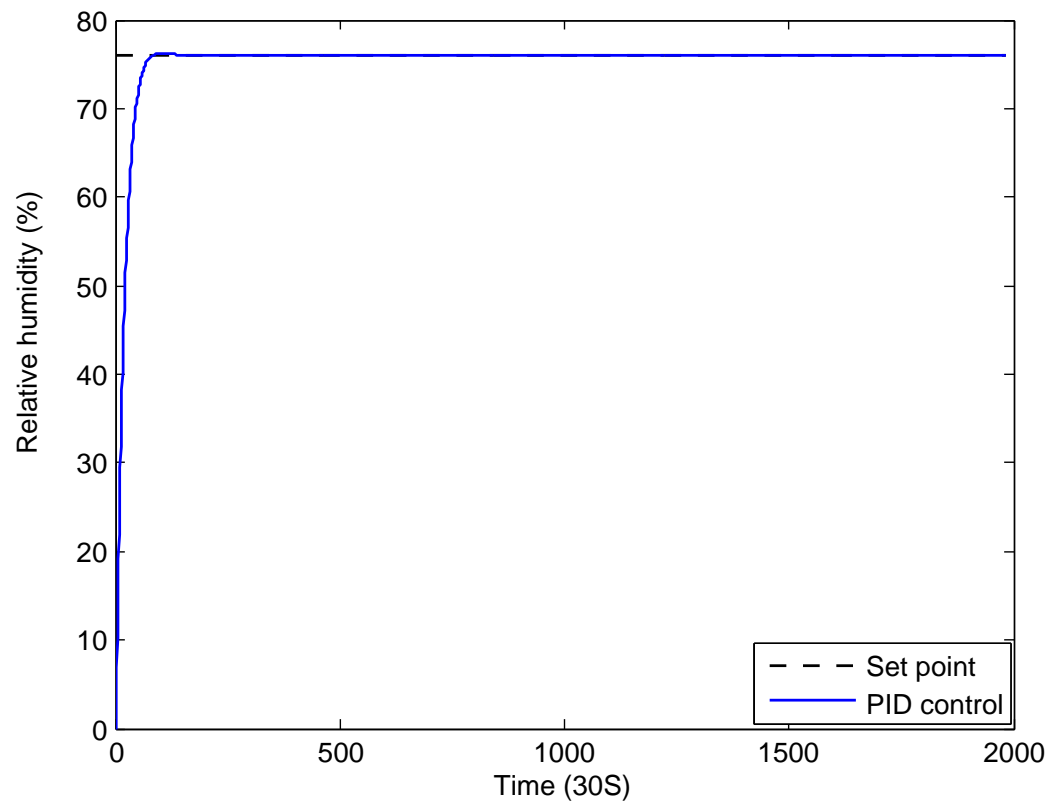

Figure 5.3: The output of PID control without relative humidity disturbances. 


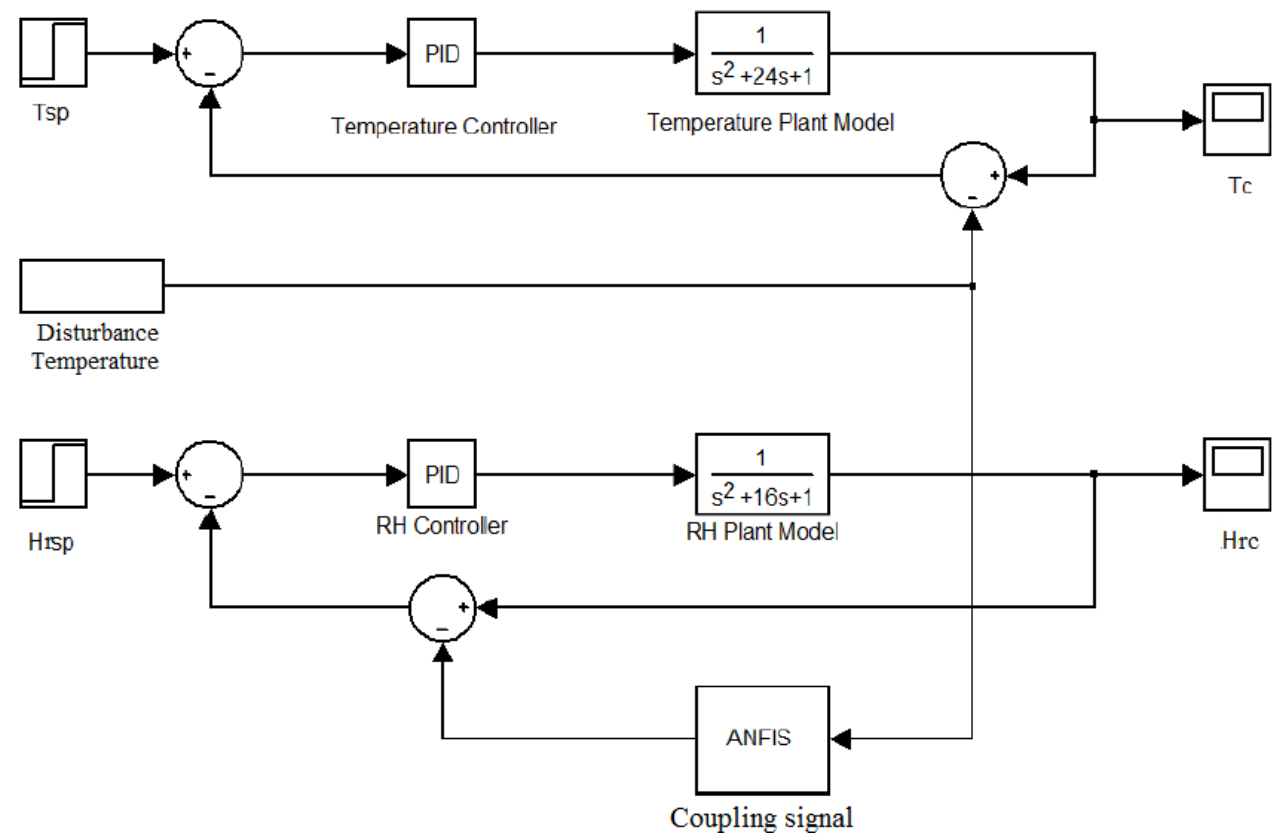

Figure 5.4: The block diagram of PID control with disturbances.

\section{PID control with disturbances}

A disturbance signal of temperature was added into the temperature control loop, which simulated the PID control in disturbance conditions. Another model of a disturbance signal of the coupling was added into the relative humidity control loop, which simulated the PID control in the coupling effects. The purpose of adding two disturbance signals is to simulate the control systems of the meat drying room, which is a more realistic way of describing a real meat drying system. Fig. 5.4 shows a block diagram of simulation systems with a Simulink tool in Matlab. Figs. 5.5 and 5.6 show results of temperature and relative humidity that add the two disturbance signals into closed-loop PID control systems.

As shown in Fig. 5.6, the input is a step unit, and the set point of relative humidity is 76 $\%$. The output of relative humidity is in a range from $75.2 \%$ to $77.1 \%$ with disturbances. The solid line is the simulation output of PID control. Thus, the PID controller was hardly tuned. However, the error is still approximately $2 \% \mathrm{RH}$. Therefore, the PID controller of relative humidity does not perform well to reduce disturbances of the coupling. 


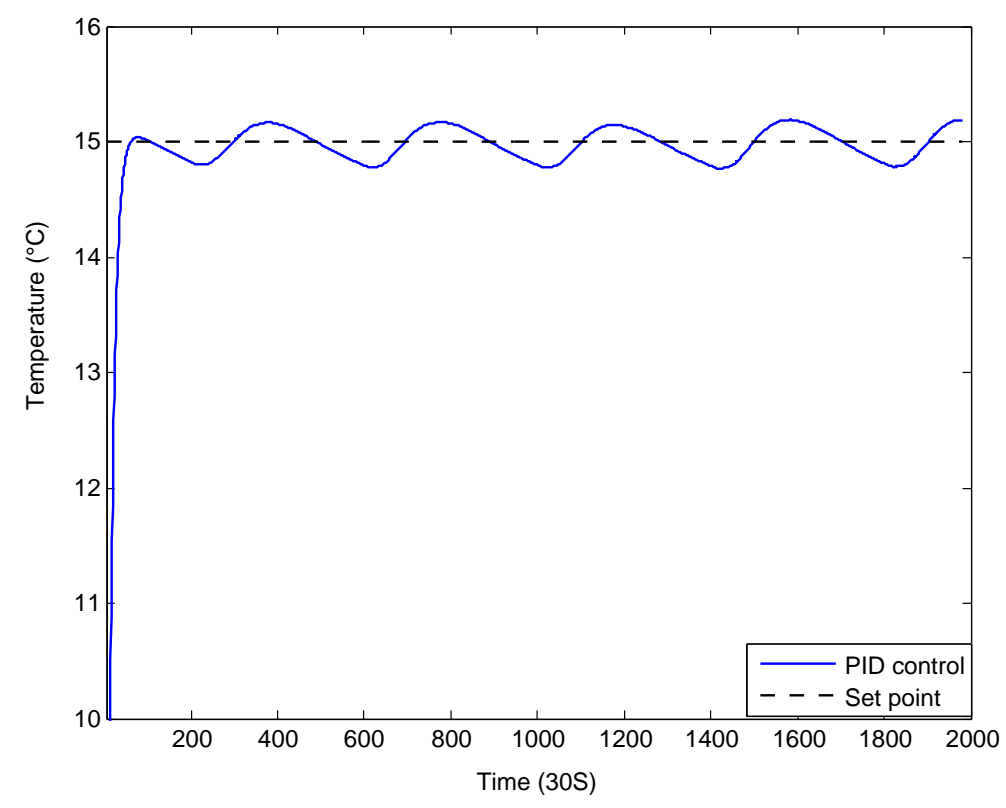

Figure 5.5: The simulation results of PID control with disturbances for temperature.

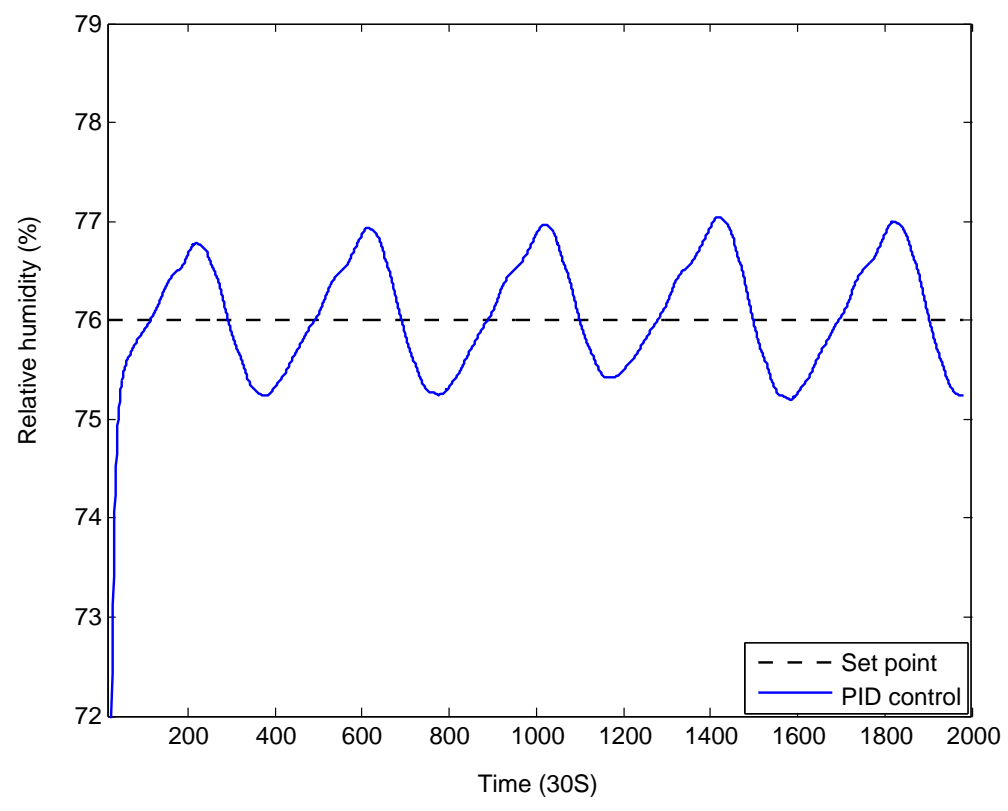

Figure 5.6: The simulation results of PID control with disturbances for relative humidity. 


\section{Fuzzy PID control}

As the above section discussed, the result of the coupling shows a feature of nonlinear fluctuations. The model of the coupling used an ANFIS method in Matlab. Therefore, the fuzzy PID control method was selected to overcome the nonlinear problem in this research.

Due to external environment effects in a meat drying room, the coupling issue cannot be modeled with classical precise mathematical equations and may fluctuate in an uncertain manner. As a consequence of this, the suitable model of the fuzzy control system must behave like a skilled human.

The proposed fuzzy PID control is a fuzzy logic controller added into the relative humidity control loop in order to improve the control performance of systems and eliminate the effect of coupling.

In this thesis, the input of the fuzzy model was an error (E), which was a set-point signal minus a feedback signal. The Mamdani-type fuzzy inference was used in the fuzzy model for relative humidity control loop. The range of overall inputs $\mathrm{E}_{\text {ih }}$ was [-1 0.5]. Fig. 5.7 shows the inputs of the fuzzy model. The output of the fuzzy model was following rules and Mamdani fuzzy inferences; the output connected to the PID controller. The range of overall outputs $\mathrm{E}_{\text {oh }}$ was [-0.8 0.5]. Fig. 5.8 shows the outputs of the fuzzy model.

The structure of the fuzzy PID control with a block diagram is shown in Fig. 5.9, and the simulations of fuzzy PID control uses Simulink tool in Matlab.

The range of the inputs and the range of outputs were set up by a designer. The membership functions were selected for inferences and rules, which was the triangle algorithm method for this fuzzy model. As shown in Fig. 5.10, the input of relative humidity control is a step unit, and a set point of relative humidity is $76 \% \mathrm{RH}$. The dash line is an output of PID control, and the solid line is an output of fuzzy PID control simulations. The error of PID control is still approximately 4\% RH. The error of fuzzy PID control is almost $-0.2 \%$ RH. Comparing the results of the fuzzy PID control simulation and real PID control, the fuzzy PID control shows a better control accuracy than PID control. 


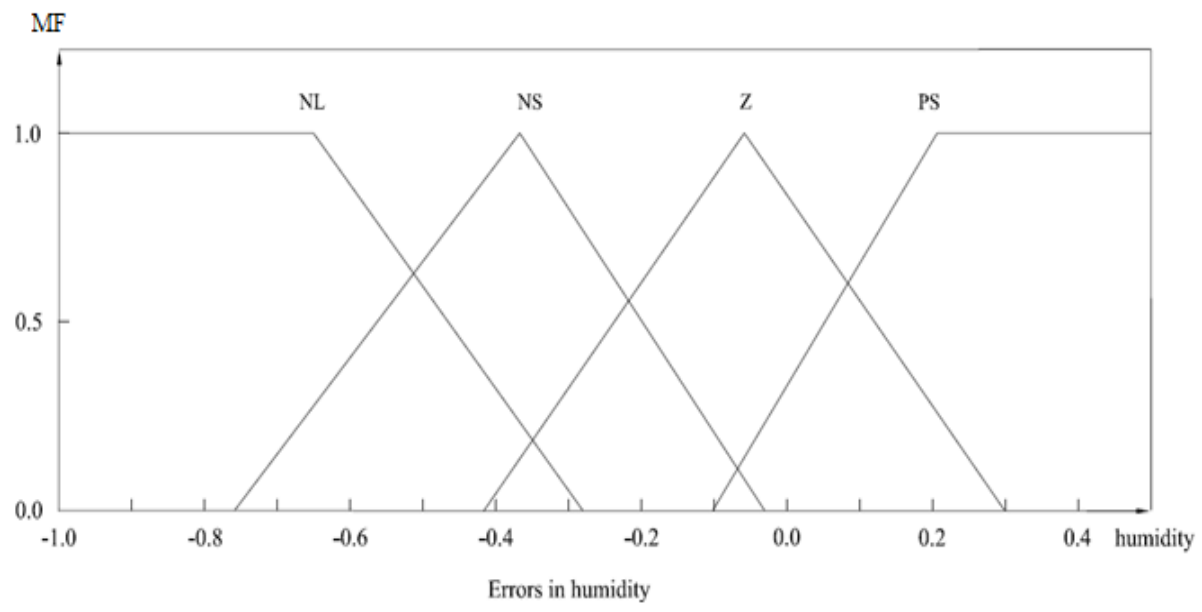

Figure 5.7: The input membership functions of the fuzzy controller.

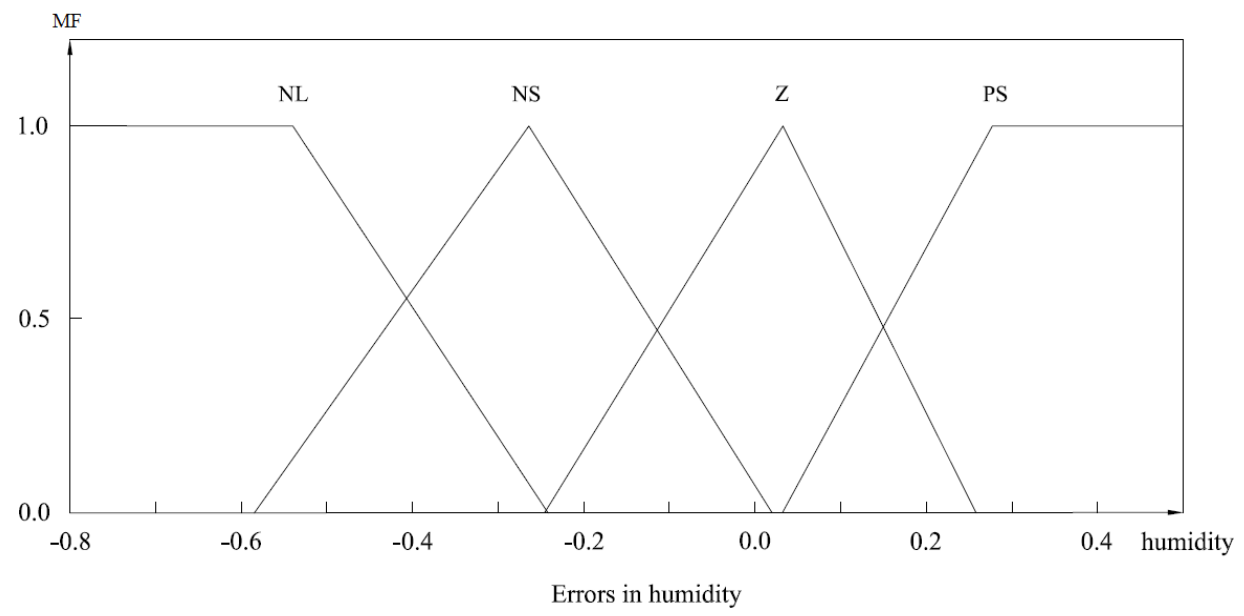

Figure 5.8: The outputs of membership functions of the fuzzy controller. 


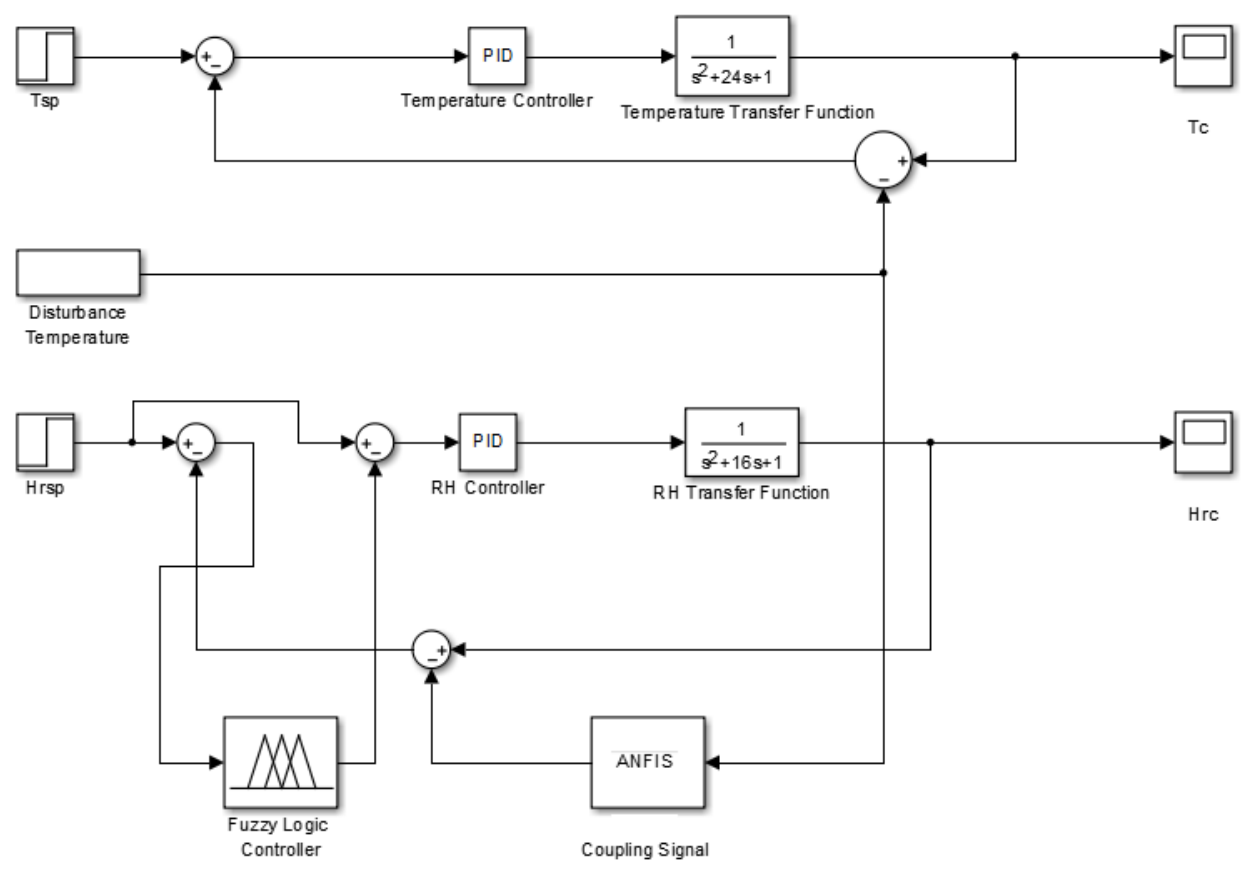

Figure 5.9: The block diagram of fuzzy PID control.

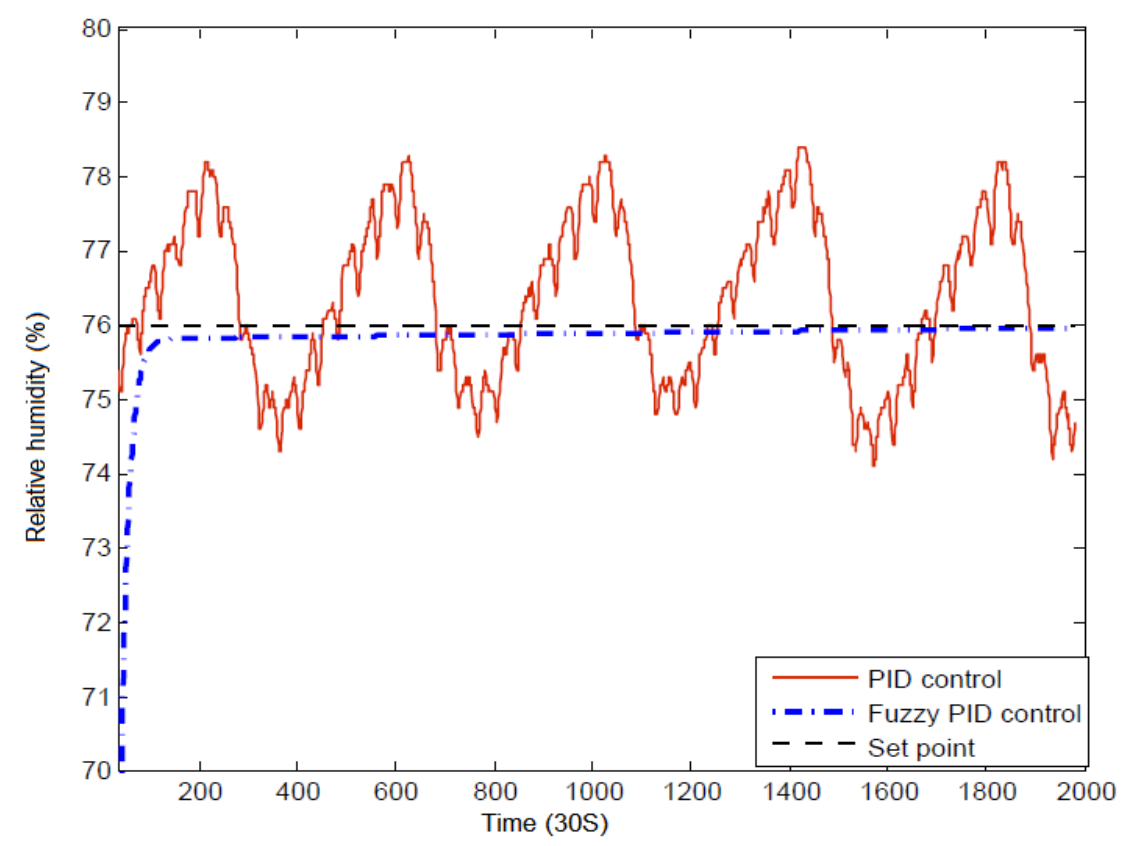

Figure 5.10: The outputs of fuzzy PID control and PID control for RH. 


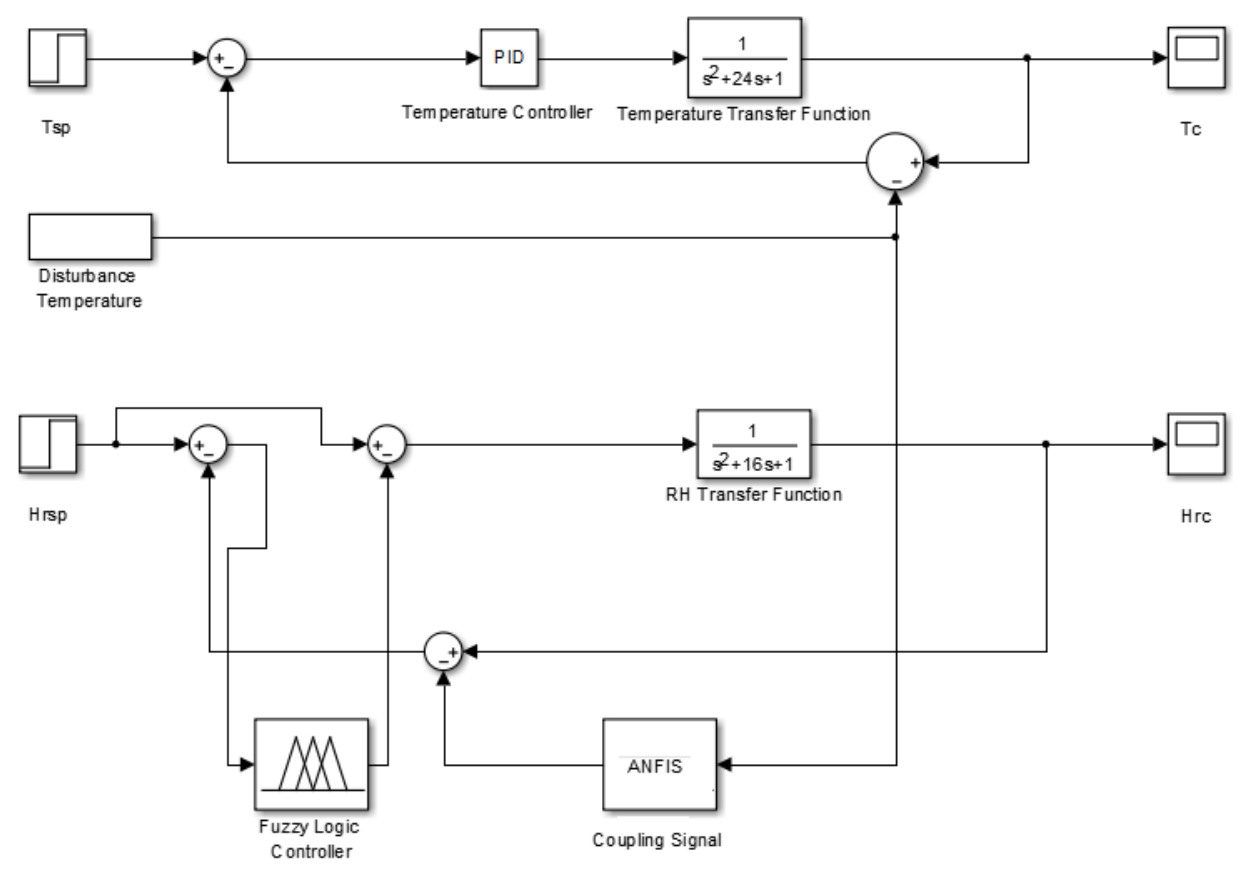

Figure 5.11: The block diagram of fuzzy control for relative humidity.

\section{Fuzzy logic control}

As the above result shown, the fuzzy PID control method used for control a meat drying room. Next method will be fuzzy logic control. The block diagram of fuzzy logic control is shown in Fig. 5.11. A fuzzy logic controller added into the relative humidity control loop. For the relative humidity control loop, a fuzzy controller in a fuzzy control model was designed as the same as the fuzzy controller in a fuzzy PID control model. The experiments of the fuzzy control were conducted with a Simulink tool in Matlab.

The range of the inputs and the range of outputs were set up by a designer. The membership functions were selected for inferences and rules, which was the triangle algorithm method for this fuzzy model. As shown in Fig. 5.12, the input of relative humidity control is a step unit, and the dash line is a set point of relative humidity at $76 \%$. The solid line is the output of PID control, and the dash-dot line is the output of fuzzy control. The error of PID control is still $4 \% \mathrm{RH}$. The error of fuzzy control is almost $0.2 \% \mathrm{RH}$. Comparing the results of the fuzzy control and PID control, the fuzzy control shows a better control accuracy than PID control. 


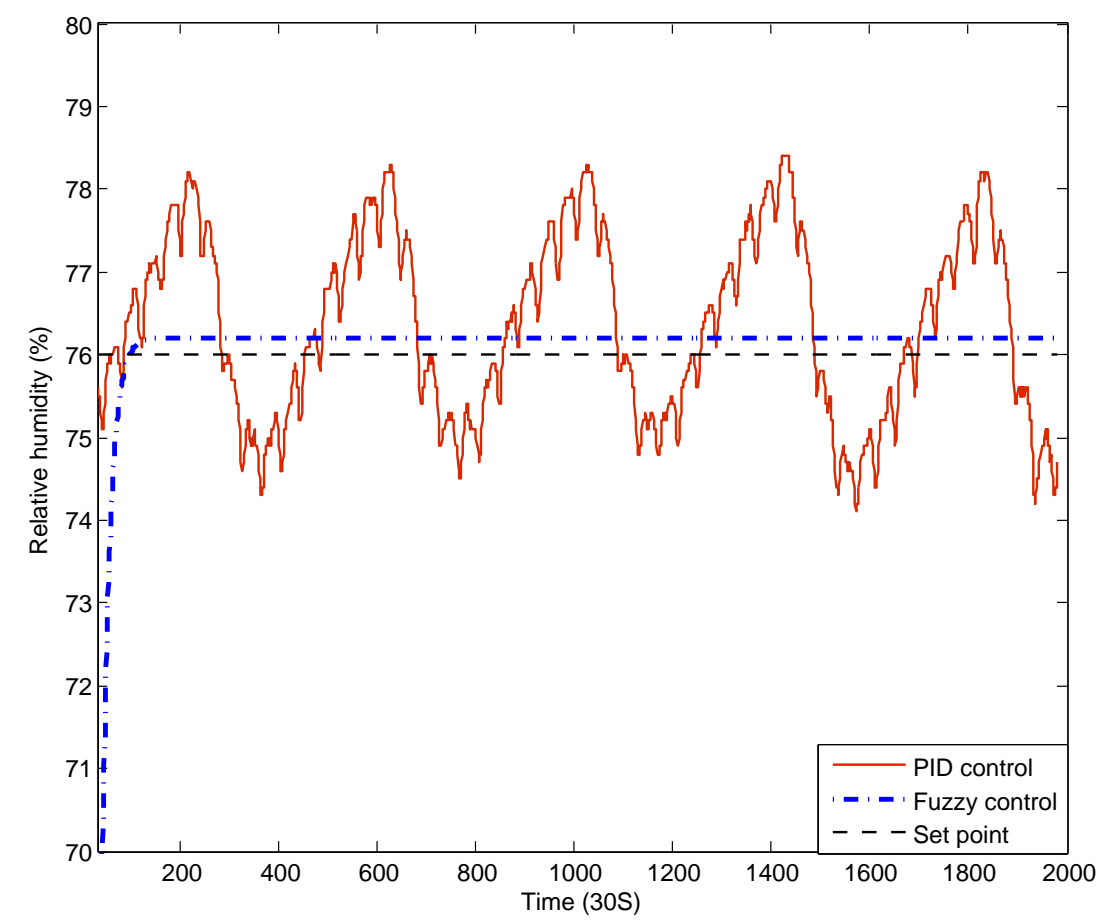

Figure 5.12: The outputs of fuzzy control and PID control for relative humidity.

\subsubsection{Digital Control Systems}

Analog control systems as the above discussions are improved the meat drying room control systems. Digital control systems including a temperature control loop and a relative humidity control loop will be next. Control methods in digital control systems will show a different results and improments.

\section{PI control in discrete-time}

Proposed PI controllers in discrete-time was designed for a temperature controller and a relative humidity controller. The block diagram of digital PI control is shown in Fig. 5.13. The experiments of digital PI control were conducted in Matlab. Figs. 5.14 and 5.15 show the results of tmeperature and relative humidity in digital PI control.

As shown in Fig. 5.15, the input of relative humidity control is a step unit, and a set point of relative humidity is $76 \%$. The dash line is an a set point, and the solid line is the output of digital PI control. The error of digital PI control is still approximately $1.8 \% \mathrm{RH}$. Comparing the results of the digital PI control and analog PID control for relative humidity, 


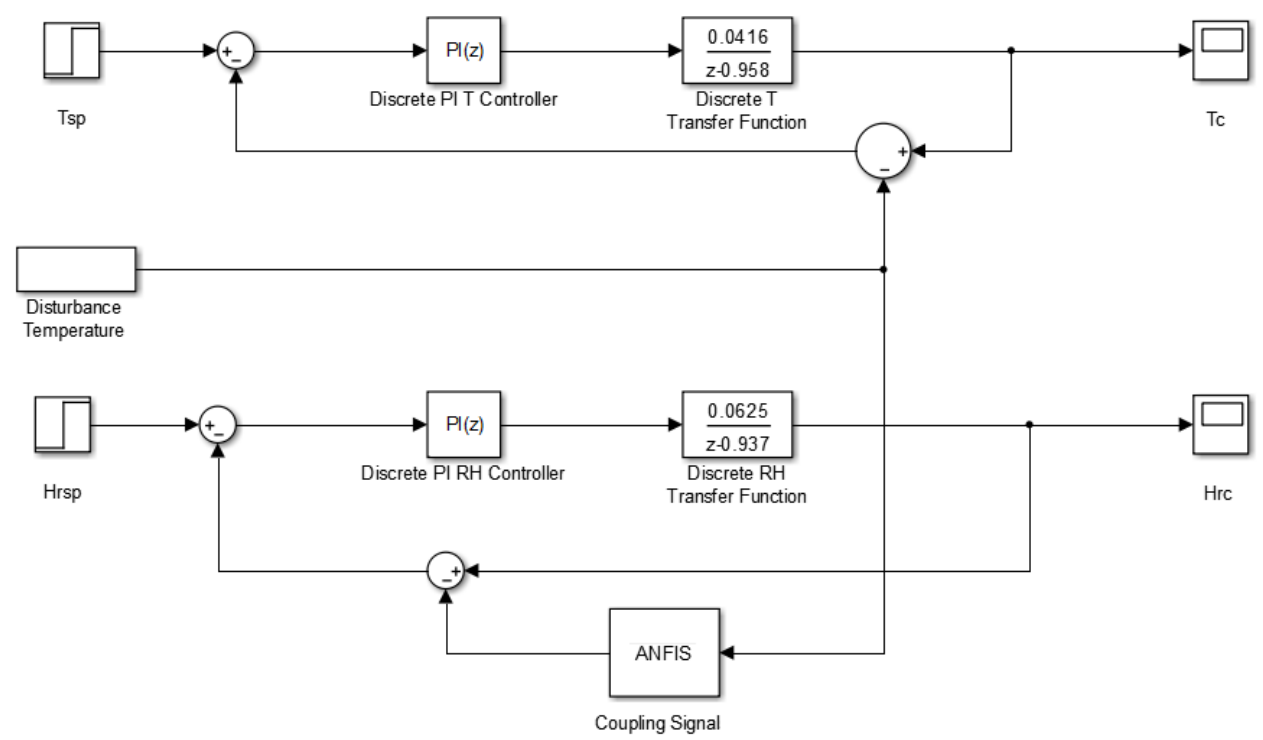

Figure 5.13: The block diagram of digital PI control for temperature and RH.

control accuracy is no big difference.

\section{PI-like fuzzy control}

Proposed digital control systems include a temperature control loop and a relative humidity control loop. A block diagram of the digital control system for the meat drying room is shown in Fig. 5.16. In Fig. 5.16, where $T_{s p}$ is the set point of temperature, $H_{r s p}$ is the set point of relative humidity, $T_{c}$ is the system output of the temperature, $H_{r c}$ is the system output of the relative humidity. The temperature control loop has a feedback control which includes a digital PI controller, a disturbance signal, a fuzzy controller, and a plant model. Proposed control input of the temperature loop is a single point control that has a desired point as a constant input. The relative humidity control loop has a feedback control that includes a digital PI controller, a disturbance signal, , a fuzzy controller, and a plant model. Proposed control input of the relative humidity is a single point control that has a desired point as a constant input.

Digital PI-like fuzzy logic controller was designed to write a program in an $\mathrm{m}$ code script in MatLab. A structure of the programming flow chart is shown in Fig. 5.17. 


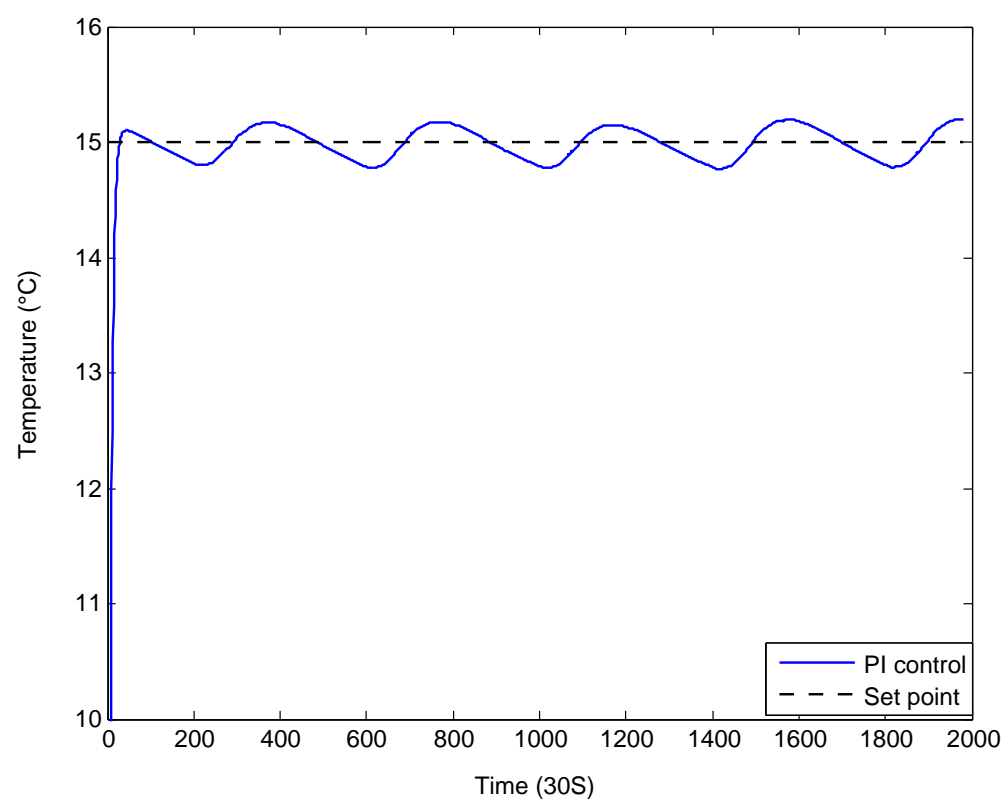

Figure 5.14: The output of digital PI control for temperature.

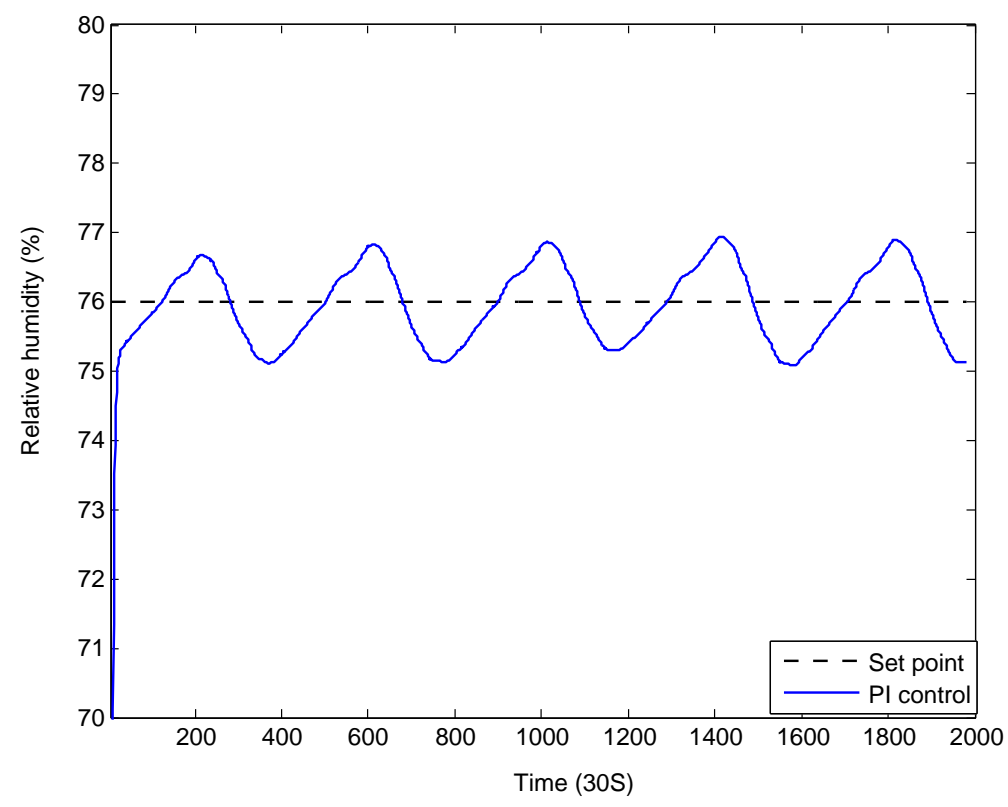

Figure 5.15: The outputs of digital PI control for RH control. 


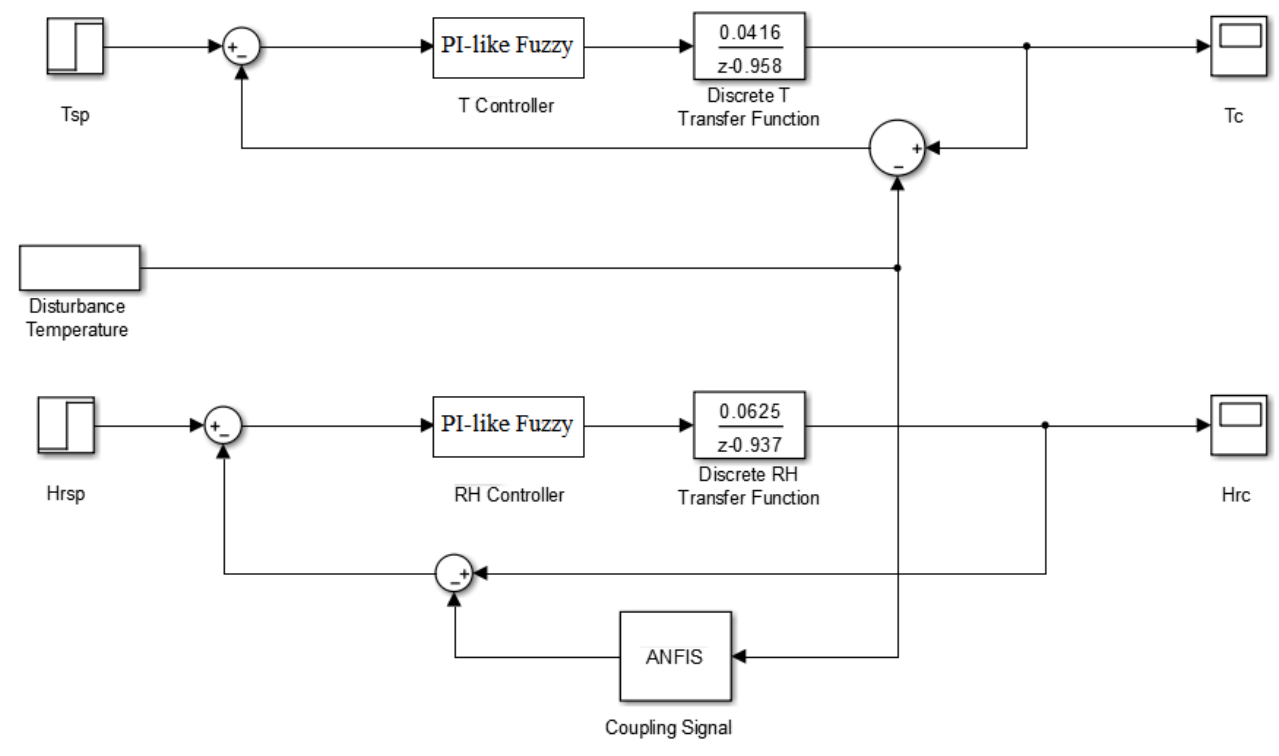

Figure 5.16: The block diagram of digital control for a meat drying room.

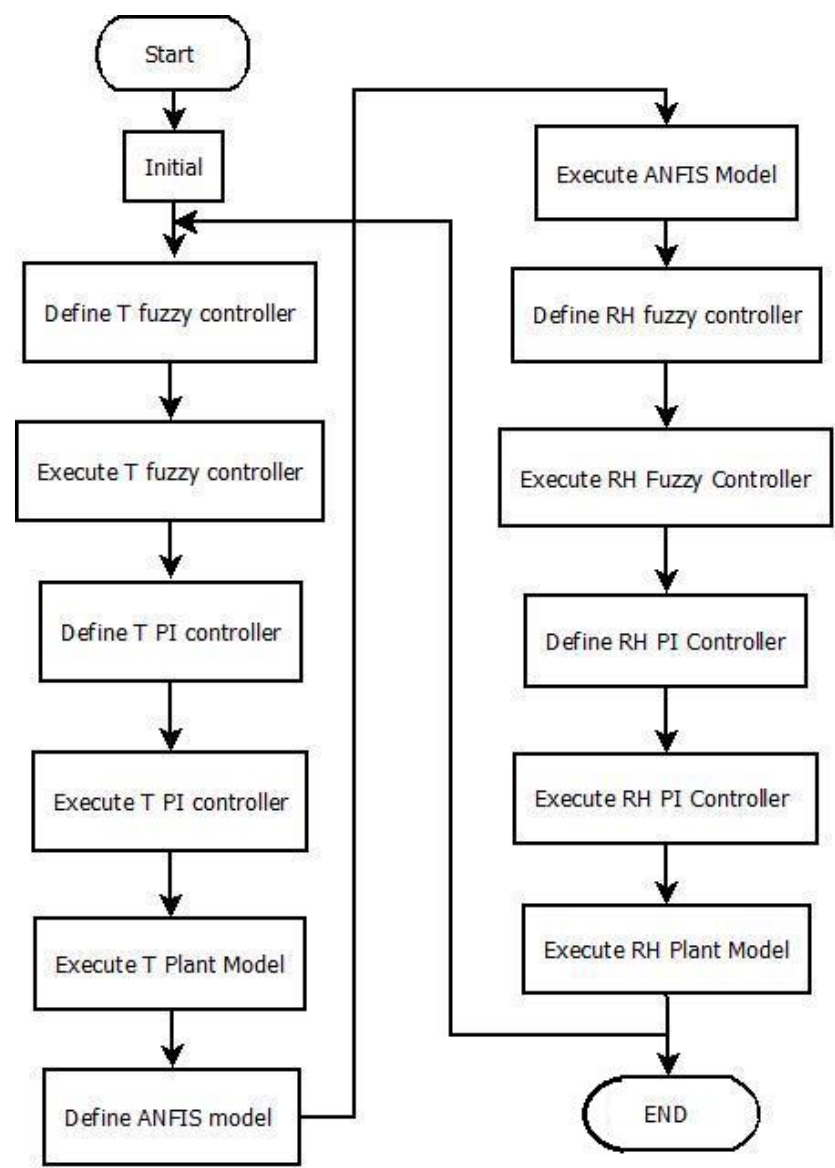

Figure 5.17: The flow diagram of the digital control systems. 


\section{Design digital control systems of temperature}

The fuzzy logic controller of temperature is two inputs and one output. The structure of the fuzzy controller programming is given as

1. System name is given.

2. A temperature input variable $\mathrm{e}_{i t}$ is defined and given the fuzzy inference system within a range [ $-0.20 .2]$.

3. A temperature input variable $\Delta \mathrm{e}_{i t}$ is defined and given the fuzzy inference system within a range [- 0.20 .2 ].

4. A temperature output variable $\mathrm{e}_{o t}$ is defined and given the fuzzy inference system within a range $\left[\begin{array}{ll}-0.1 & 0.1\end{array}\right]$.

5. Rules are set up within three input membership functions for two input variables and three output membership functions for one output variable.

6. The fuzzy logic controller ends and connects to digital PI controller.

The input membership functions of the fuzzy temperature controller are shown in Figs. C.1 and C.2. The output membership functions of the fuzzy temperature controller are shown in Fig. C.3.

The digital PI controller of temperature is an input $u_{t}(t)$ and an output $T_{c}(t)$. The structure of the digital PI controller programming is:

1. Initialize with the parameters $r_{0 t}, r_{1 t}$, and $T_{s t}$.

2. Set up the desired signal.

3. Obtain errors of temperature with a loop of index $k_{t}$.

4. Obtain proportional and integral algorithms.

5. Sum a proportional output and an integral output and the last value of an input into current value. 
6. The PI-like fuzzy controller ends and connects to the digital system of the plant model.

The digital model of the coupling was added with the digital temperature control system after the digital PI controller. The digital system of the plant model was created in Chapter 3. A complete digital control system of temperature for a meat drying room used a closed loop feedback control system to have the output controlled at a desired temperature. A tuning of the temperature PI controller was to change the parameters, $r_{o t}$ and $r_{1 t}$. The parameters $a_{1 t}$ and $b_{1 t}$ were obtained for transfer functions from chapter 3 . The final equations of the control systems are as follows.

The transfer function of temperature in the continuous-time model is obtained as Equation (3.13), so the parameters $r_{0 t}$ and $r_{1 t}$ can be calculated for the digital PI controller as follows.

$$
r_{0 t}=G_{t}\left(1+\frac{T_{s t}}{T_{t}}\right)=1 \times\left(1+\frac{0.5}{24}\right)=1.021
$$

and

$$
r_{1 t}=-G_{t}=-1
$$

Substituting $r_{0 t}$ and $r_{1 t}$ into Equation (5.5), $u_{t}(t)$ is given as

$$
u_{t}(t)=u_{t}(t-1)-1.021 y_{t}(t)+T c(t-1)+1.021 r_{t}(t)-r_{t}(t-1) .
$$

The output $y(t)$ of temperature in the closed loop control system is given as

$$
T c(t)=\frac{0.0416}{z-0.958} u_{t}(t)+d_{t}(t) .
$$

The complete programming code includes calculation equations and sequence of the programming. The simulation result of the PI-like fuzzy logic control of temperature is shown in Fig. 5.18.

\section{Digital control systems of relative humidity}

The fuzzy logic controller of relative humidity has two inputs and one output. The structure of the fuzzy controller programming is:

1. System name is given. 


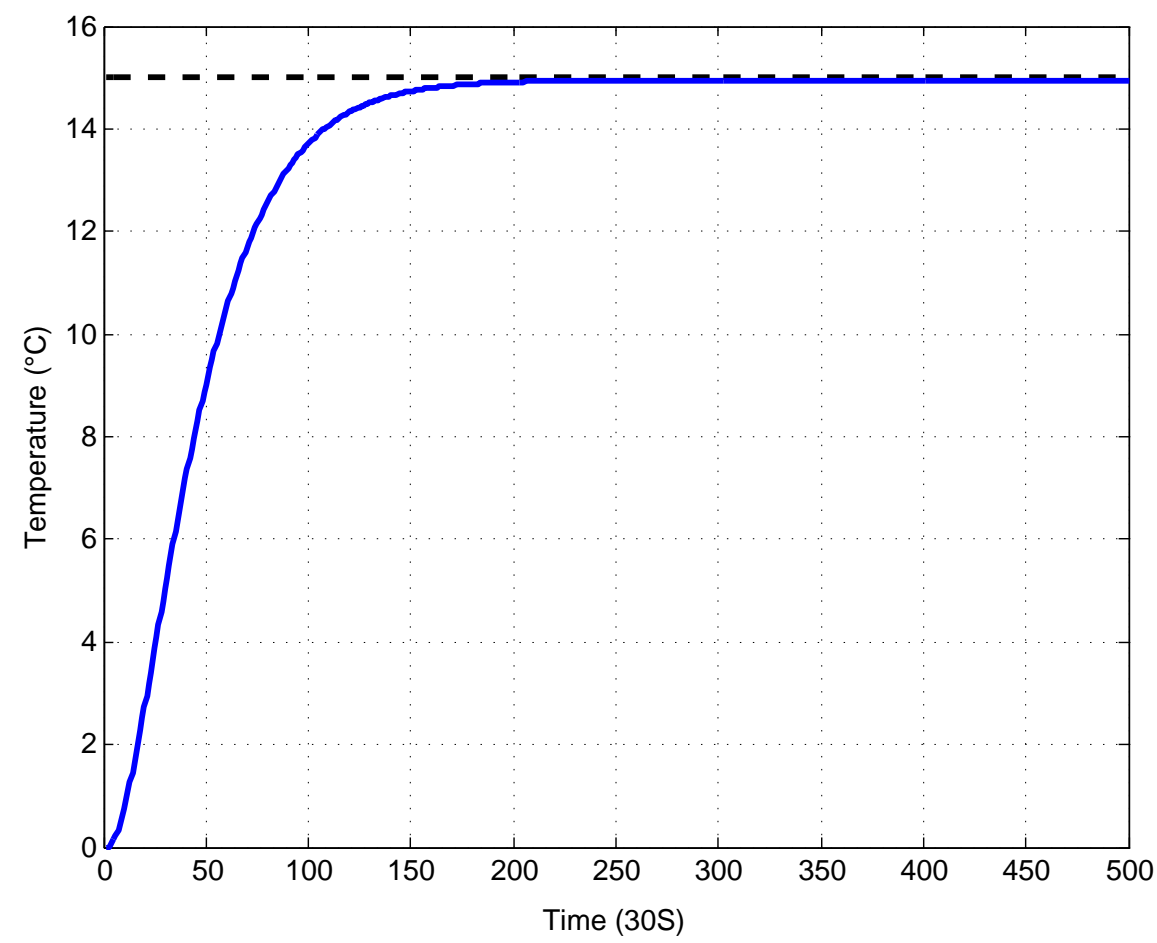

Figure 5.18: The temperature output of digital control for a drying room system.

2. A relative humidity input variable $\mathrm{e}_{i h}$ is defined and given the fuzzy inference system within a range [-1 1$]$.

3. A relative humidity input variable $\Delta \mathrm{e}_{i h}$ is defined and given the fuzzy inference system within a range [-0.2 0.2].

4. A relative humidity output variable $\mathrm{e}_{o h}$ is defined and given the fuzzy inference system within a range $\left[\begin{array}{ll}-1 & 0.5\end{array}\right]$.

5. Rules are set up with three input membership functions for two input variables and three output membership functions for one output variable.

6. The fuzzy logic controller ends and connects to digital PI controller.

The input membership functions of the fuzzy relative humidity controller are shown in Figs. C.4 and C.5. The output membership functions of the fuzzy relative humidity controller are shown in Fig. C.6. 
The digital PI controller of relative humidity has an input $u_{h}(t)$ and an output RHc(t). The steps of simulation are:

1. Initialize with the parameters of $r_{0 h}, r_{1 h}$, and $T_{s h}$.

2. Set up the desired signal.

3. Obtain errors of temperature with a loop of index $k_{h}$.

4. Obtain proportional and integral algorithms.

5. Sum a proportional output and an integral output and the last value of an input into current value.

6. Return to the beginning.

The digital model of the coupling for the relative humidity is created using ANFIS model. The structure of the digital ANFIS model is:

1. Training data of temperature and relative humidity is prepared and loaded into the workspace.

2. Set up ANFIS parameters, number of membership functions, type of membership functions, and number of epochs.

3. ANFIS structure is created and the training data is tested.

4. The training data is evaluated as the output data of relative humidity.

5. Coupling model ends and outputs to the control systems.

The digital model of the coupling was added into the control system of relative humidity. The digital system of the relative humidity plant was created in Chapter 3. A complete digital control system of relative humidity for a meat drying room used a closed loop feedback control system to have a control output at a desired signal of relative humidity. A tuning of the digital relative humidity PI controller was to change the parameters, $r_{o h}$ and 
$r_{1 h}$. The parameters $a_{1 h}$ and $b_{1 h}$ were obtained for transfer functions from section 3 . The final equations of control systems are as follows.

The transfer function of relative humidity in a continuous-time model is obtained as Equation (3.14), so the parameters $r_{0 h}$ and $r_{1 h}$ can calculate the PI controller as follows.

$$
r_{0 h}=G_{h}\left(1+\frac{T_{s h}}{T_{h}}\right)=1 \times\left(1+\frac{0.5}{16}\right)=1.031,
$$

and

$$
r_{1 h}=-G_{h}=-1
$$

Substitute $r_{0 h}$ and $r_{1 h}$ into Equation (5.13), $u_{h}(t)$ is given as

$$
u_{h}(t)=u_{h}(t-1)-1.031 y_{h}(t)+H r c(t-1)+1.031 r_{h}(t)-r_{h}(t-1) .
$$

The output $y_{h}(t)$ of relative humidity in the closed loop control system is given as

$$
\operatorname{Hrc}(t)=\frac{0.0625}{z-0.937} u_{h}(t)+d_{h}(t)
$$

The complete programming includes calculation equations and sequence of the programming. The simulation result of the PI-like fuzzy logic control for relative humidity is shown in Fig. 5.19.

\subsection{Comparison of the Control Methods}

The performance of the proposed relative humidity controllers in this section was compared. The comparison encompasses the methods of PID control, fuzzy PID control, fuzzy control, discrete PI control, and PI-like fuzzy-control, as shown in Table 5.4. Comparing the error values shown in Table 5.4, the digital PI-like fuzzy control method gives the best control accuracy. The control accuracy improvement for a meat drying room not only improves the quality meat products, but also reduces the energy cost of operating the temperature and relative humidity control systems. Normally, temperature and relative humidity control systems use low-pressure steam, low-temperature glycol, and water with air pressure as mediums to control temperature and relative humidity in a meat drying room. Both equipment (steam generator systems and glycol-ammonia systems) are high energy cost 


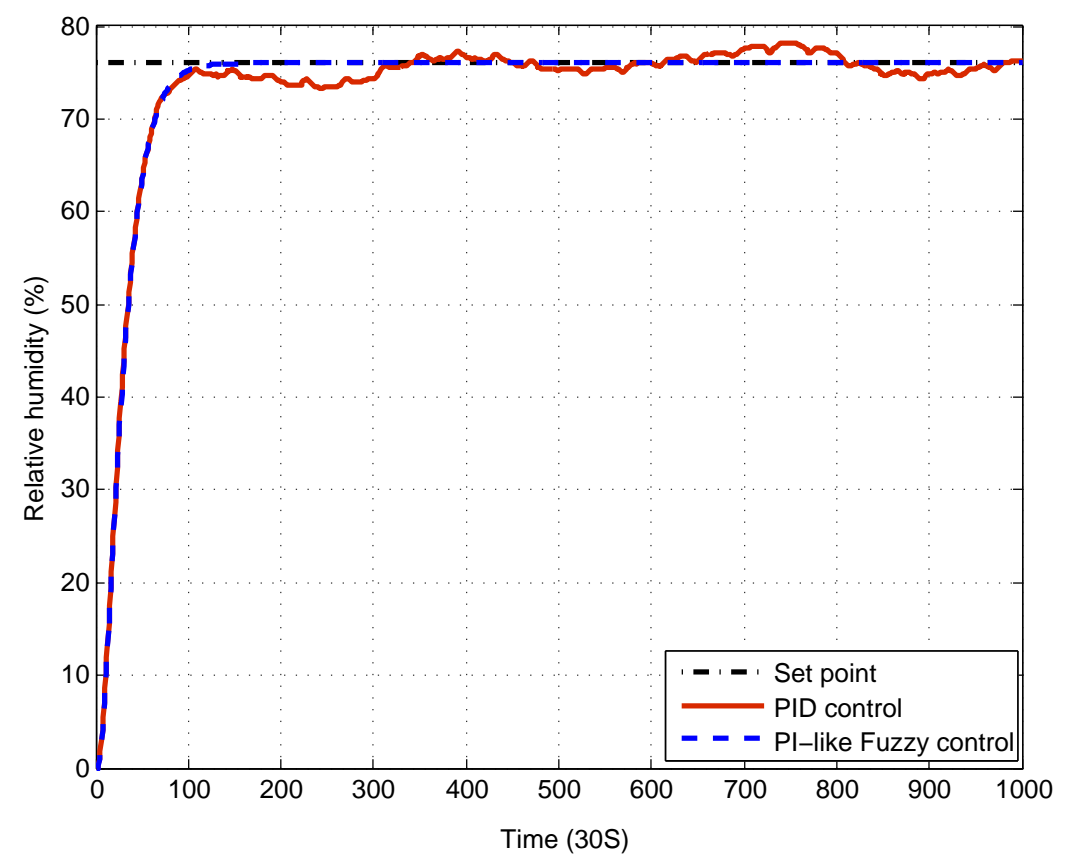

Figure 5.19: The digital control of relative humidity for a drying room system.

installations. If control accuracy of temperature and relative humidity systems is higher, the energy cost will be lower.

Table 5.4: Comparison of relative-humidity control performances.

\begin{tabular}{|c|c|c|c|}
\hline Analog Control & & Digital Control & \\
\hline Methods & Error (\%RH) & Methods & Error (\%RH) \\
\hline PID & 2 & PI & 1.5 \\
\hline Fuzzy PID & -0.2 & PI-like fuzzy & 0 \\
\hline Fuzzy & 0.2 & PID-like fuzzy & N/A \\
\hline
\end{tabular}

\subsection{Summary}

This chapter proposed control methods for a meat drying room system and developed models for simulations in both continuous-time and discrete-time. The controllers were designed based on PID, fuzzy PID, fuzzy, discrete PI, and PI-like fuzzy control for the relative humidity control, which eliminated the coupling signal and showed very good con- 
trol performance. Comparing the results of fuzzy PID, fuzzy, or PI-like fuzzy control with real-life measured PID control, the fuzzy PID, fuzzy, or PI-like fuzzy-control showed better control accuracy than discrete-PI control and PID control. Therefore, a fuzzy-based control method is well-suited for the control of meat drying systems. 


\section{Chapter 6}

\section{Conclusions and Future Works}

In this thesis, a complete intelligent control system of a meat drying room was developed. The plant model of the drying room was obtained in the form of temperature and relative humidity transfer functions. The coupling model of the drying room was obtained using an ANFIS model. The controller of temperature was designed using a PID algorithm in continuous-time domain and a PI-like fuzzy method in discrete-time domain. The controller of relative humidity was designed using a fuzzy PID control method in continuoustime domain and a PI-like fuzzy method in discrete-time domain. The PI-like fuzzy control was shown to achieve better accuracy.

\subsection{Conclusions}

Transfer functions of temperature and relative humidity for a meat drying room were obtained. Real measured data of step responses for temperature and relative humidity were obtained from a meat drying room. The transfer function models were proposed with a first-order system in both continuous-time and discrete-time domains as well as a secondorder system in continuous-time domain. Simulated step responses of temperature and relative humidity closely matched the measured step responses of temperature and relative humidity. Gain and time constant parameters in the transfer functions were adjusted to best-fit the measured step responses of temperature and relative humidity.

The coupling model of temperature and relative humidity was obtained and analyzed. 
To model the coupling, real data were obtained from a drying room. The coupling model was based on ANFIS. The relationship between temperature and relative humidity was found to be nonlinear. The model was applied to a simulated drying room control system and verified to closely match measured coupling from a real drying room.

The complete intelligent control system of a meat drying room was developed. The intelligent controllers were designed to overcome the issue of coupling in a drying room system, which was found to be nonlinear. The controllers were proposed using the fuzzy PID method and the PI-like fuzzy method. It was found that the control method of the fuzzy PID in continuous-time domain has an offset and very small errors, and the output of relative humidity with the fuzzy PID control takes longer to reach the set point. The PI-like fuzzy control in discrete-time domain was found to be better than the fuzzy PID control in continuous-time domain for the complete control system. The output of relative humidity with the PI-like fuzzy control reaches the set point quickly. The control fluctuation of relative humidity is very small. The control accuracy of the PI-like fuzzy method, the fuzzy method, and the fuzzy PID method is better than PID control.

\subsection{Future Works}

A topic for future work is to implement the proposed digital control system in practice. A PI-like fuzzy logic controller will be designed and programmed. The software should be easy to convert into other languages and embedded into a micro-controller. The physical control system should include sensors for temperature and relative humidity measurements, a meat drying room, and a controller. Another topic for future work is to analyze the energy savings of heating, ventilation, humidifying, dehumidifying and cooling (air conditioning) for meat drying systems running the proposed control method. The focus will be to analyze the effects of temperature and relative humidity control on the on/off time of steam valves and glycol valves to indirectly compare energy consumption with the current meat drying control system. Due to the time constraints of this research, there was not sufficient time to study methods for measuring a relationship between energy consumption and cost efficiency for a meat drying room system. 


\section{References}

Arabinda, K. P. (2008). Development of neuro-fuzzy controller for applications to hvac system, inverted pendulum and other processes. International Journal of Computational Cognition 6(2), 585-598.

Arcidiacono, G. (2014). Scientific calculators: Correlation coeffient calculator. http://www.alcula.com/calculators/statistics/correlation-coefficient/.

Argüello-Serrano, B. and M. Vélez-Reyes (1999). Nonlinear control of a heating, ventilating, and air conditioning system with thermal load estimation. IEEE Transactions on Control Systems Technology 7(1), 56-63.

Arnau, J., L. Guerrero and P. Gou (1997). Effect of temperature during the last month and salting time on dry-cured hams aged for six months. Journal of the Science of Food and Agriculture 74(2), 193-198.

Arnau, J., P. Gou and J. Comaposada (2003). Effect of the relative humidity of drying air during the resting period on the composition and appearance of dry-cured ham surface. Journal of Meat Science 65(4), 1275-1280.

Åström, K. J. and R. M. Murray (2008). Feedback Systems: A Introduction for Scientists and Engineering. Princeton University Press. Princeton, USA.

Baucour, P. and J. D. Daudin (2000). Development of a new method for fast measurement of water sorption isotherms in the high humidity range validation on gelatine gel. Journal of Food Engineering 44(2), 97-107. 
Becker, M., D. Oestreich, H. Hasse and L. Litz (1994). Fuzzy control for temperature and humidity in refrigeration systems. In: Proceedings of the Third IEEE conference on Control Applications. Glasgow, UK. pp. 1607-1612.

Bi, Q., W. Cai, Q. Wang, C. Hang, E. Lee, Y. Sun, K. Liu, Y. Zhang and B. Zou (2000). Advamced controller auto-tuning and its application in hvac system. Control Engineering Practice 8(6), 633-644.

Bon, J., G. Clemente, J. A. Cárcel and A. Mulet (2005). Management and optimization of curing chambers. Journal of Food Engineering 68, 33-41.

Cai, C. L., Z. H. Fu, K. Ren, N. Yu and S. C. Chai (2011). Design of intelligent dry and wet bulb humidity sensor. Instrument Technique and Sensors 40(1), 8-10.

Caponetto, R., L. Fortuna, G. Nunnari, L. Occhipinti and M. G. Xibilia (2000). Soft computing for greenhouse climate control. IEEE Transactions on Fuzzy Systems 8(6), 753760.

Chi, P., F. Weng, A. Su and S. Chan (2006). Numerical modeling of proton exchang membrane fuel with considering thermal and relative huidity effects on the cell performance. ASME Journal Fuel Cell Science Technology 3(3), 292-303.

Chiang, M. L. and L. C. Fu (2006). Hybrid system based adaptive control for the nonlinear hvac system. In: Proceedings of American Control Conference. Minneapolis, USA. pp. 5324-5329.

Daskalov, P. I. (1997). Prediction of temperature and humidity in a naturally ventilated pig building. Agricultural Engineering Research 68(4), 329-339.

Daskalov, P. I., K. G. Arvanitis, G. D. Pasgianos and N. A. Sigrimis (2006). Non-linear adaptive temperature and humidity control in animal building. Biosystems Engineering 93(1), 1-24.

Dorf, R. C. and R. H. Bishop (2008). Modern Control Systems. Prentice -Hall. Upper Saddle River, USA. 
Feyissa, A. H., K. V. Gernaey, S. Ashokkumar and J. Adler-Nissen (2011). Modelling of coupled heat and mass transfer during a contact baking process. Journal of Food Engineering 106(3), 228-235.

Fisher, P. D., S. L. Lillevik and A. L. Jones (1981). Microprocessors simplify humidity measurements. IEEE Transactions on Instrumentation and Measurement 30(1), 57-63.

Gou, P., J. Comaposda, E. Serra, M. Corominas, M. Poch and J. Arnau (2005). Fuzzy control system in drying process of fermented sausages. Drying Technology 23(9-10), 20552069.

Haykin, S. (2009). Neural Networks and Learning Machines. Pearson Education Inc. New Jersey, USA.

Hertog, M. L. A. T. M., R. Ben-Arie, E. Róth and B. M. Nicolaï (2004). Humidity and temperature effects on invasive and non-invasive firmness measures. Postharvest Biology and Technology 33(1), 79-91.

Hoang, M. L., P. Verboven, M. Baelmans and B. M. Nicolaï (2004). Sensitivity of temperature and weight loss in the bulk of chicory roots with respect to process and product parameters. Journal of Food Engineering 62(1), 233-243.

Hu, Q. L., J. Cao, L. P. Sun and Y. Q. Liu (2009). Neural network based robust variable structure control of wood drying kiln. In: Proceedings of Chinese Control and Decision Conference. Guilin, China. pp. 4828-4833.

Jahedi, G. and M. M. Ardehali (2012). Wavelet based artificial neural network applied for energy effciency enhancement of decoupled hvac system. Energy Conversion and Management 54, 47-56.

Jang, J. R. (1993). Anfis: Adsptive-network-based fuzzy inference system. IEEE Transactions on systems 23(3), 665-685.

Landau, I. D. and G. Zito (2006). Digital Control Systems. Springer. London, UK.

Leistner, L. (1986). Allgemeinesüber rohschinken. Fleischwirtschaft 66(4), 496-510. 
Li, J., D. Yan and R. Qu (2012). The strategy of construction equipment energy-saving control. Telkomnika Indonesian Journal of Electrical Engineering 10, 706-712.

Lin, S. X. Q. and X. D. Chen (2005). A effective laboratory air humidity generator for drying research. Journal of Food Engineering 68(1), 125-131.

Linker, R., M. Kacira and A. Arbel (2011). Robust climate control of a greenhouse equipped with variable-speed fans and a variable-pressure fogging system. Biosystems Engineering 110(2), 153-167.

Liu, H. and J. H. Xu (2008). Research hybrid fuzzy-pid control technology based on the temperature and humidity control. In: Proceedings of International Symposium on Computational Intelligence and Design. Wuhan, China. pp. 190-193.

Liu, X. W. and T. F. Da (2011). Design for fuzzy decoupling control system of temperature and humidity. In: Proceedings of Advanced Research on Computer Science and Information Engineering. Zhengzhou, China. pp. 231-236.

Ma, H., W. Zhang and S. X. Yang (2013). Coupling analysis and control of temperature and relative humidity in a drying room. In: Proceedings of IEEE International Conference on Infomation and Automation. Ningxia, China. pp. 1381-1385.

Mahajan, P. V., F. A. R. Oliveira and I. Macedo (2008). Effect of temperature and humidity on the transpiration rate of the whole muchrooms. Journal of Food Engineering 84(2), 281-288.

Mahmud, S. (2013). Air-Conditioning Lecture Notes. University of Guelph. Guelph, Canada.

Mannheim, C. H., J. X. Lui and S. G. Gilbert (1994). Control of water in foods during storage. Journal of Food Engineering 22, 509-532.

Mathioulakis, E., G. Panaras and V. Belessiotis (2011). Estimation of uncertainties in indirect humidity measurements. Journal of Energy and Buildings 43(10), 2806-2812.

MSTE (2015). The power of squares. http://mste.illinois.edu/patel/amar430/meansquare.html. 
Munoz, I., J. Comaposda, J. Stawczyk and P. Pou (2007). Drying control of cured sausages through online measurement of product quality. Drying Technology 25(11), 1809-1817.

Nakanishi, E., N. C. Pereira, L. T. Fan and C. L. Hwang (1973). Simultaneous control of temperature and humidity in a confined space. Building Science 8(1), 51-64.

Nantou, Y. (1979). Digital ventilated psychrometer. IEEE Transactions on Instrumentation and Measurement 28(1), 42-45.

Nassif, N., K. Stanislaw and R. Sabourn (2005). Optimization of hvac control system strategy, using two objective genetic algorithm. International Journal of HVAC \& R Research 11(3), 459-486.

Nathakaranakule, A., W. Kraiwanichkul and S. Soponronnarit (2007). Comparative study of different combined superheated-steam drying techniques for chicken meat. Journal of Food Engineering 80(4), 1023-1030.

Neiva, A. C. D., B. E. D. Reis and C. G. Sanchez (2006). Calibration and validation of a new aspirated psychrometer for technological development of a humidifier. In: Processdings of 18th World Congress on Metrology for a Sustainable Development. Rio de Janeiro, Brazil. pp. 1-6.

Oliver, F. J. (1971). Practical Instrumentation Transducers. Hayden. New York, USA.

Ondier, G. O., T. J. Siebenmorgen and A. Mauromoustakos (2010). Low-temperature, lowrelative humidity drying of rough rice. Journal of Food Engineering 100(3), 545-550.

Parolari, G., R. Virgili and C. Schivazappa (1994). Relationp between cathepsin b activity and compositional parameters in drycured hams of normal and defective texture. Meat Science 38(1), 117-122.

Pasgianos, G. D., K. G. Arvanitis, P. Polycarpou and N. Sigrimis (2003). A nonlinear feedback technique for greenhouse environmental control. Computers and Electronics in Agriculture 40(1-3), 153-177. 
Powell, R. W. (1936). The use of thermocouples for psychrometric purposes. Journal of the physical society 48(3), 406-414.

Prachayawarakorn, S., S. Sawangduanpen, S. Saynampheung, T. Poolpatarachewin, S. Soponronnarit and A. Nathakarakule (2004). Kinetics of colour change during storage of dried garlic slices as affected by relative humidity and temperature. Journal of Food Engineering 62(1), 1-7.

Rentel-Gómez, C. and M. Vélez-Reyes (2001). Decoupled control of temperature and relative humidity using a vjariable-air-volume hvac system and non-interacting control. In: Proveedings of the 2001 IEEE International Conferce on Cotrol Applications. Mexico City, Mexico. pp. 1147-1151.

Riverol, C. and C. D. Sanctis (2009). A fuzzy filter for improving the quality of the signal in adaptive-network-based fuzzy inference systems (anfis). Applied Soft Computing 9(1), 305-307.

Salazar, R., I. López and A. Rojano (2007). A neural network model to predict temperature and relative humidity in a greenhouse. In: Processdings of International Symposium on High Technology for Greenhouse System Management. Naples, Italy. pp. 539-546.

Schirmer, M., W. B. Hussein, M. Jekle, M. A. Hussein and T. Becker (2011). Impact of air humidity in industrial heating processes on selected quality attributes of bread rolls. Journal of Food Engineering 105(4), 647-655.

Sebastian, P., D. Bruneau, A. Collignan and M. Rivier (2005). Drying and smoking of meat: heat and mass transfer modeling and experimental analysis. Journal of Food Engineering 70(2), 227-234.

Shi, X. M. and Z. Q. Hao (2008). Fuzzy Control and MATLAB Simulation. Tsinghua University. Beijing, China.

Siddique, N. and H. Adeli (2013). Computational and Intelligence. John Wiley \& Sons Ltd. New Jersey, USA. 
Soyguder, S. and H. Alli (2009a). An expert system for the humidity and temperature control in hvac system using anfis and optimization with fuzzy modeling approach. Energy and Buildings 41(8), 814-822.

Soyguder, S., M. Karakose and H. Alli (2009). Design and simulation of self-tuning pidtype fuzzy adaptive control for an expert hvac system. Expert Systems with Applications 36(3), 4566-4573.

Srzednicki, G. S., R. Hou and R. H. Driscoll (2006). Development of a control system for in-store drying of paddy in northeast china. Journal of Food Engineering 77(2), 368-377.

Stawczyk, J., I. Munoz, C. Collell and J. Comaposda (2009). Control system for sausage drying based on on-line nir $a_{w}$ determination. Drying Technology 27(12), 1338-1343.

Stawczyk, J., J. Comaposda, P. Gou and J. Arnau (2004). Fuzzy control system for a meat drying process. Drying Technology 22(1-2), 259-267.

Talei, A., L. H. C. Chua and T. S. W. Wong (2010). Evaluation of rainfall and discharge inputs used by adaptive network-based fuzzy inference systems (anfis) in rainfallrunoff modeling. Journal of Hydrology 391(3-4), 248-262.

Wang, J., D. An, C. Zhang and Y. Jing (2009). Genetic optimization algorithm of pid decoupling control for vav air-conditioning system. Transation Tianjin University 15(1), 308314.

Wang, J., Q. M. Xu and T. G. Qin (2006). Application of a fuzzy decoupling control algorithm for simulaneous control of both temperature and humidity. In: Proceedings of The SPIE International Society For Optical Engineering. Urumqi, China. pp. 1-6.

Wang, J., Y. Jing and C. Zhang (2008). Genetic optimization algorithm on pid decoupling controller for variable flow heating system. In: Proceedings of 8th IEEE conference on Industrial Electronic and Applications. Singapore, Singapore. pp. 510-515.

Zhang, C. F., J. He and X. Q. Zhu (2004). Reasearch of intelligent humidity control system based on field-bus. In: Proceedings of 8th International conference on Control, Automation, Robotics and Vision. Kunming, China. pp. 2145-2149. 
Zhang, D. Y., Y. Q. Liu and J. Cao (2003). Application of single neuron adaptive pid controller during the process of timber drying. Journal of Forestry Research 14(3), 244-248.

Zhang, W. and S. X. Yang (2010). A fuzzy based approch to effecttive relative humidity tranking in industrial systems. In: Proceedings of 8th World Congress on Intelligent Control and Automation. Jinan, China. pp. 2148-2153. 


\section{Appendix A}

\section{Relative Humidity Sensors}

This Appendix presents relative humidity sensors in Chapter 2. 


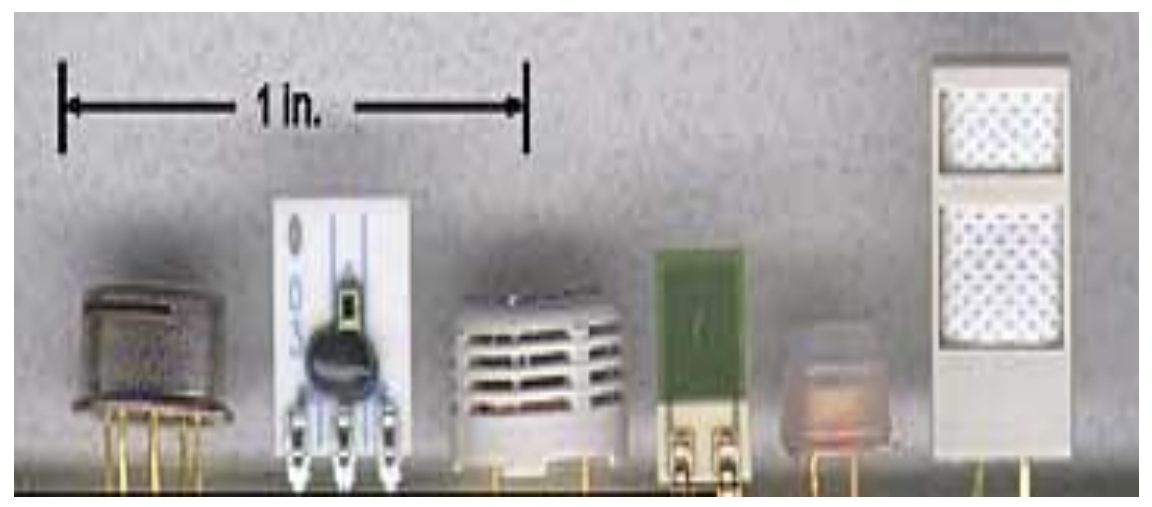

Figure A.1: Capacitive RH sensors. (from: http://itp.nyu.edu/physcomp/sensors/Reports/SHT11)

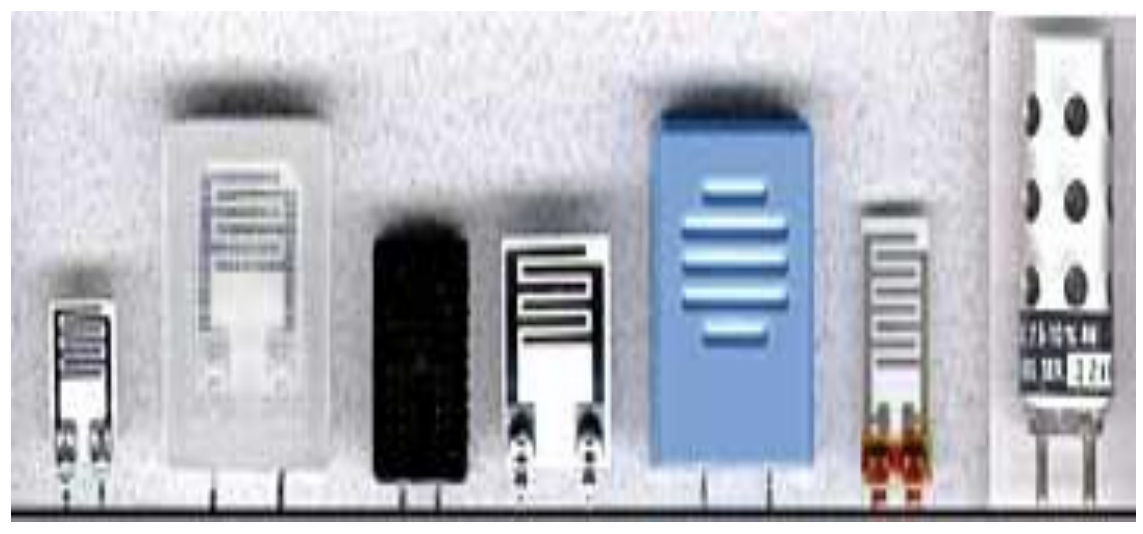

Figure A.2: Resistive RH sensors. (from: http://itp.nyu.edu/physcomp/sensors/Reports/SHT11) 


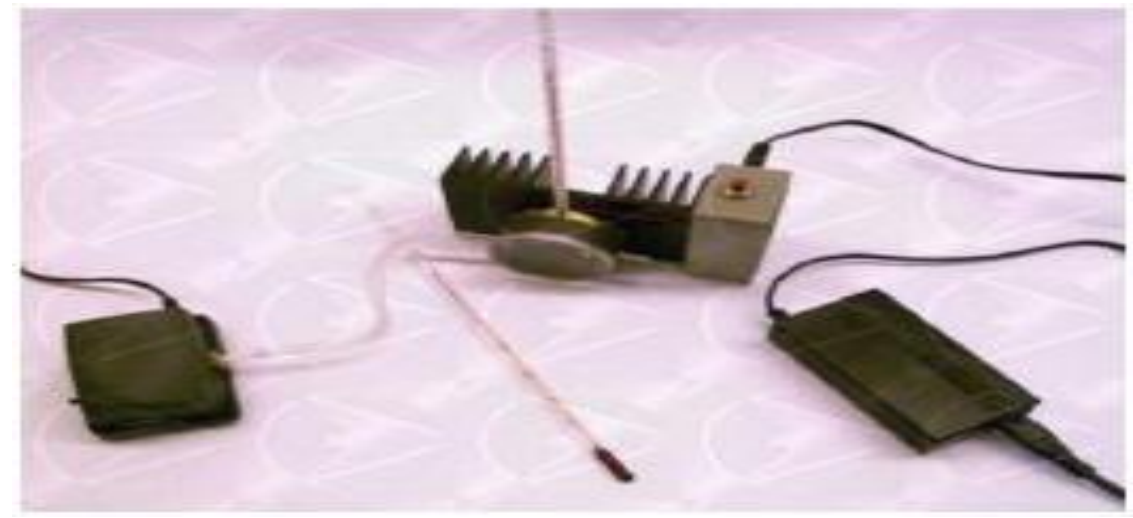

Figure A.3: Dew point hygrometers. (from: http://www.alfarez.com/frames/hilton/heattransfer2.html)

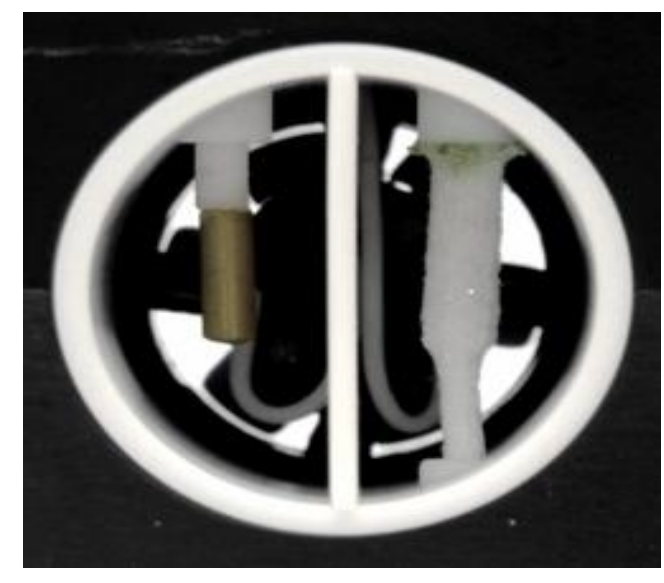

Figure A.4: The structure of a psychrometer (from: http://www.itc.gov.hk/en/quality/scl/hm/- hm3.htm). 


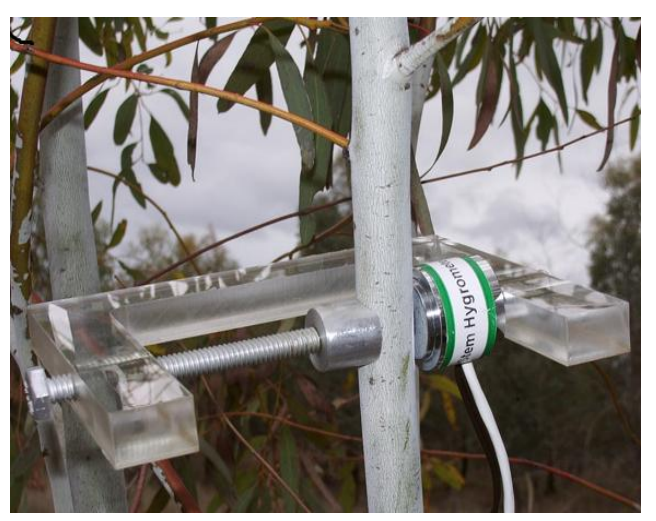

Figure A.5: An application of a hygrometer. (from: http://www.ictinternational.com/m/psy1.html) 
Appendix B

\section{Real Data of a Drying Room}

This Appendix presents the real data in Chapter 3. 
Table B.1: The real data of temperature for a drying room system

\begin{tabular}{|c|c|c|c|c|c|c|c|}
\hline Index & Temperature $(\mathrm{C})$ & Index & Temperature $(\mathrm{C})$ & Index & Temperature(C) & Index & Temperature(C) \\
\hline 1 & 0.1 & 31 & 2.2 & 61 & 2.8 & 91 & 3 \\
\hline 2 & 0.2 & 32 & 2.2 & 62 & 2.8 & 92 & 3 \\
\hline 3 & 0.3 & 33 & 2.3 & 63 & 2.9 & 93 & 3 \\
\hline 4 & 0.4 & 34 & 2.4 & 64 & 2.9 & 94 & 3.1 \\
\hline 5 & 0.6 & 35 & 2.4 & 65 & 2.9 & 95 & 3.1 \\
\hline 6 & 0.6 & 36 & 2.5 & 66 & 2.9 & 96 & 3.1 \\
\hline 7 & 0.7 & 37 & 2.5 & 67 & 2.9 & 97 & 3.1 \\
\hline 8 & 0.7 & 38 & 2.5 & 68 & 2.9 & 98 & 3 \\
\hline 9 & 0.8 & 39 & 2.5 & 69 & 2.9 & 99 & 3 \\
\hline 10 & 0.9 & 40 & 2.5 & 70 & 2.9 & 100 & 3 \\
\hline 11 & 1 & 41 & 2.6 & 71 & 2.9 & 101 & 3.1 \\
\hline 12 & 1.2 & 42 & 2.6 & 72 & 3 & 102 & 3.1 \\
\hline 13 & 1.3 & 43 & 2.7 & 73 & 3 & 103 & 3.2 \\
\hline 14 & 1.4 & 44 & 2.7 & 74 & 3 & 104 & 3.1 \\
\hline 15 & 1.4 & 45 & 2.6 & 75 & 3 & 105 & 3.1 \\
\hline 16 & 1.4 & 46 & 2.6 & 76 & 2.9 & 106 & 3 \\
\hline 17 & 1.5 & 47 & 2.6 & 77 & 3 & 107 & 3.1 \\
\hline 18 & 1.6 & 48 & 2.7 & 78 & 3 & 108 & 3.1 \\
\hline 19 & 1.7 & 49 & 2.7 & 79 & 3 & 109 & 3.2 \\
\hline 20 & 1.8 & 50 & 2.7 & 80 & 3 & 110 & 3.2 \\
\hline 21 & 1.9 & 51 & 2.8 & 81 & 3 & 111 & 3.2 \\
\hline 22 & 1.9 & 52 & 2.7 & 82 & 3 & 112 & 3.1 \\
\hline 23 & 1.9 & 53 & 2.7 & 83 & 3 & 113 & 3.1 \\
\hline 24 & 1.9 & 54 & 2.7 & 84 & 3 & 114 & 3.1 \\
\hline 25 & 2 & 55 & 2.7 & 85 & 3 & 115 & 3.2 \\
\hline 26 & 2 & 56 & 2.8 & 86 & 3 & 116 & 3.2 \\
\hline 27 & 2.1 & 57 & 2.9 & 87 & 3.1 & 117 & 3.2 \\
\hline 28 & 2.2 & 58 & 2.9 & 88 & 3.1 & 118 & 3.2 \\
\hline 29 & 2.2 & 59 & 2.9 & 89 & 3.1 & 119 & 3.2 \\
\hline 30 & 2.2 & 60 & 2.9 & 90 & 3 & 120 & 3.2 \\
\hline
\end{tabular}


Table B.2: The real data of relative humidity for a drying room system.

\begin{tabular}{|c|c|c|c|c|c|}
\hline Index & Relative humidity & Index & Relative humidity & Index & Relative humidity \\
\hline 1 & 0.1 & 28 & 8.7 & 55 & 10.1 \\
2 & 0.3 & 29 & 8.8 & 56 & 10.1 \\
3 & 0.5 & 30 & 8.8 & 57 & 10.3 \\
4 & 2.3 & 31 & 8.7 & 58 & 10.2 \\
5 & 3 & 32 & 8.9 & 59 & 10.2 \\
6 & 3.7 & 33 & 8.8 & 60 & 10.4 \\
7 & 4.7 & 34 & 9.1 & 61 & 10.2 \\
8 & 4.2 & 35 & 9 & 62 & 10.3 \\
9 & 4.5 & 36 & 9.2 & 63 & 10.4 \\
10 & 4.5 & 37 & 9.5 & 64 & 9.9 \\
11 & 4.9 & 38 & 9.3 & 65 & 10.1 \\
12 & 5.8 & 39 & 9.4 & 66 & 10.1 \\
13 & 6 & 40 & 9.5 & 67 & 10.3 \\
14 & 6.4 & 41 & 9.4 & 68 & 10.1 \\
15 & 6.4 & 42 & 9.5 & 69 & 10.1 \\
16 & 6.6 & 43 & 9.5 & 70 & 10.1 \\
17 & 6.8 & 44 & 9.6 & 71 & 10.1 \\
18 & 6.9 & 45 & 9.7 & 72 & 10.3 \\
19 & 7.4 & 46 & 9.6 & 73 & 10.2 \\
20 & 7.7 & 47 & 9.7 & 74 & 10.2 \\
21 & 7.9 & 48 & 9.6 & 75 & 10.4 \\
22 & 8.2 & 49 & 9.9 & 76 & 10.2 \\
23 & 8 & 50 & 10.1 & 77 & 10.3 \\
24 & 8.2 & 51 & 10.1 & 78 & 10.4 \\
25 & 8.2 & 52 & 10.3 & 79 & 10.4 \\
26 & 8.3 & 53 & 10.1 & 80 & 10.4 \\
27 & 8.7 & 10.1 & & \\
\hline
\end{tabular}




\section{Appendix C}

\section{Membership Functions}

This Appendix presents the membership functions in Chapter 5. 


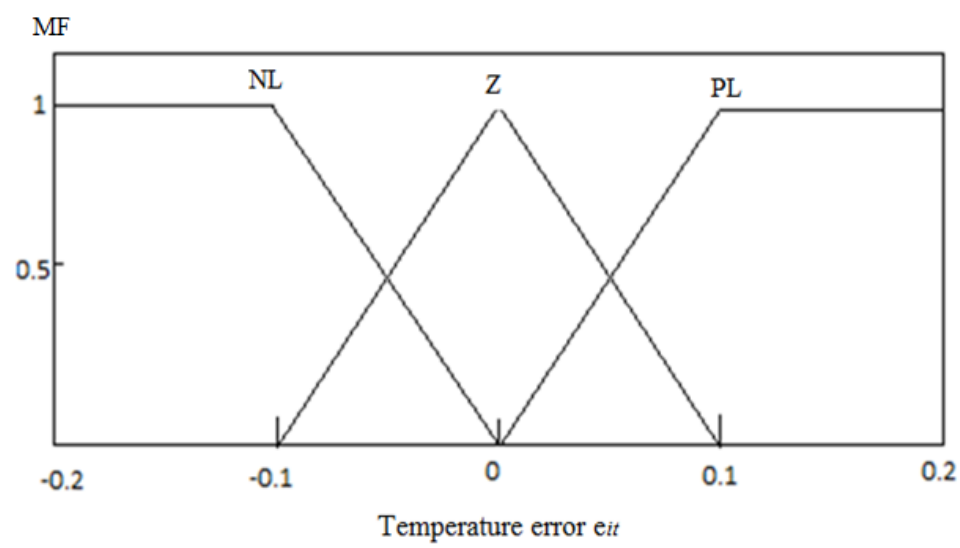

Figure C.1: Membership functions for input temperature errors.

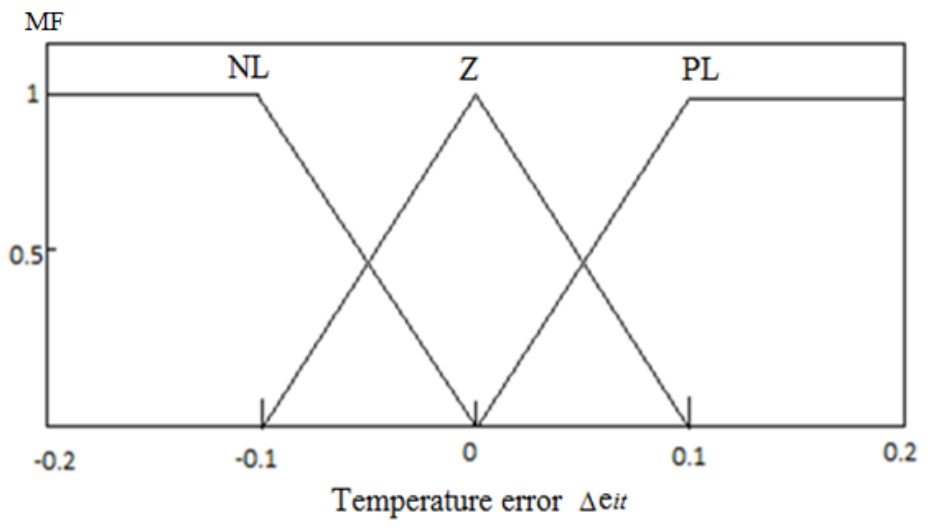

Figure C.2: Membership functions for input temperature difference errors.

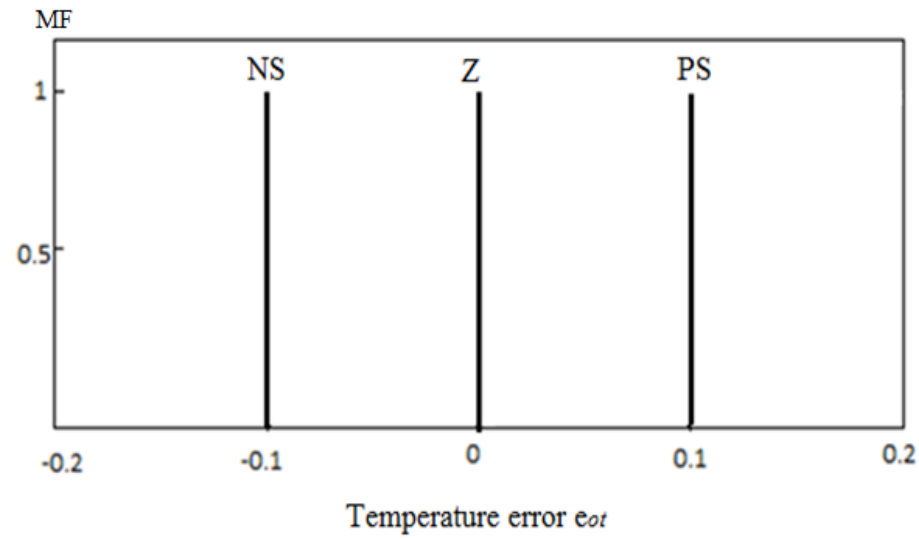

Figure C.3: Membership functions for temperature outputs. 


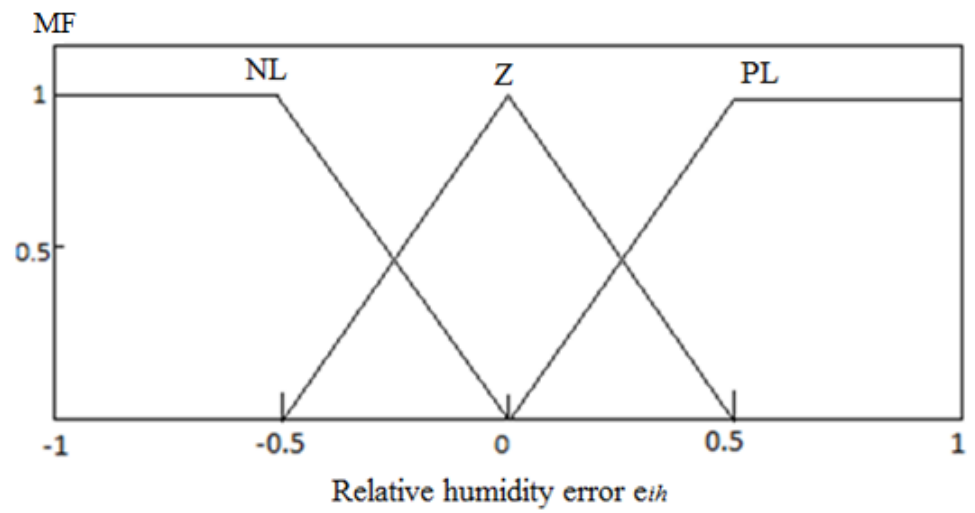

Figure C.4: Membership functions for input relative humidity errors.

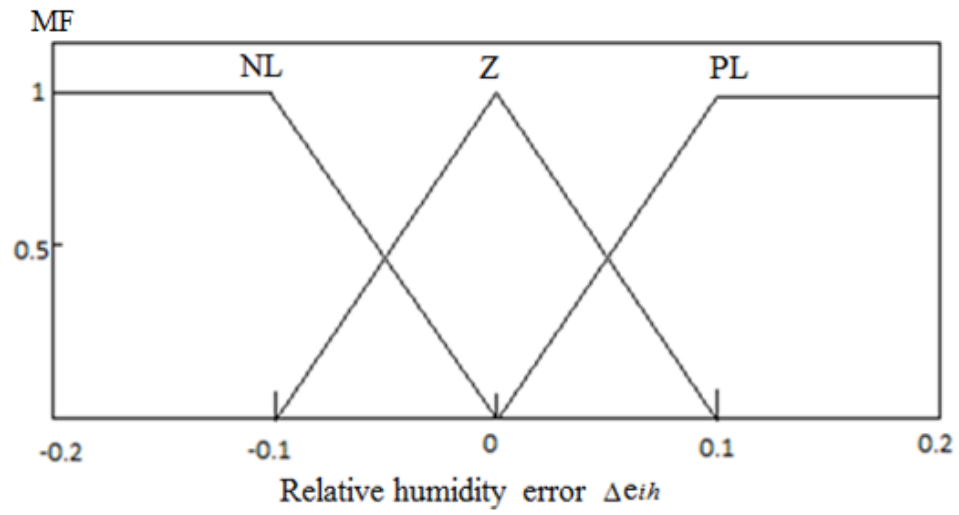

Figure C.5: Membership functions for input relative humidity difference errors.

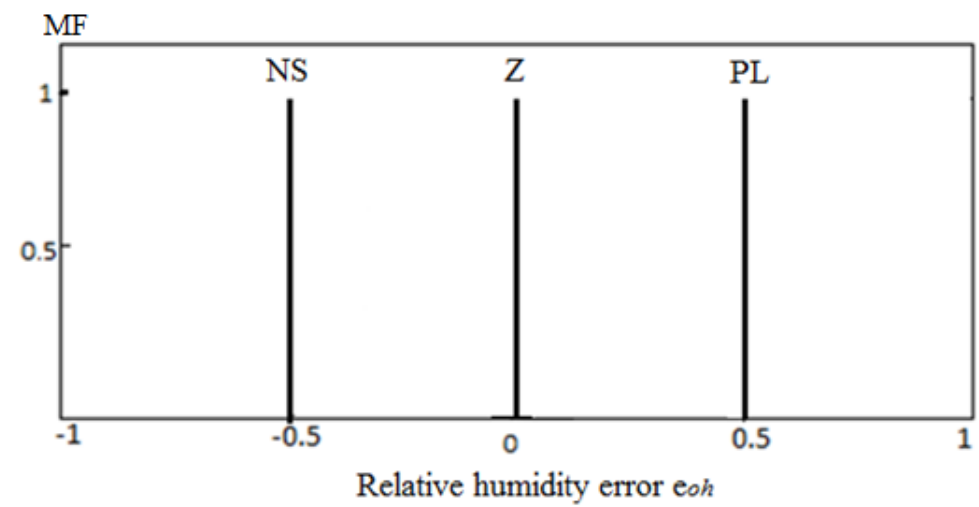

Figure C.6: Membership functions for relative humidity outputs. 